\title{
The Carnegie Supernova Project: Analysis of the First Sample of Low-Redshift Type-Ia Supernovae ${ }^{1}$
}

\author{
Gastón Folatelli ${ }^{2,3}$, M. M. Phillips ${ }^{2}$, Christopher R. Burns ${ }^{4}$, Carlos Contreras ${ }^{2}$, Mario Hamuy ${ }^{3}$, \\ W. L. Freedman ${ }^{4}$, S. E. Persson ${ }^{4}$, Maximilian Stritzinger ${ }^{2,9}$, Nicholas B. Suntzeff ${ }^{5,6}$, \\ Kevin Krisciunas ${ }^{5,6}$, Luis Boldt ${ }^{2}$, Sergio González ${ }^{2}$, Wojtek Krzeminski ${ }^{2}$, Nidia Morrell ${ }^{2}$, \\ Miguel Roth ${ }^{2}$, Francisco Salgado ${ }^{2,3}$, Barry F. Madore ${ }^{4,7}$, David Murphy ${ }^{4}$, Pamela Wyatt ${ }^{7}$, \\ Weidong $\mathrm{Li}^{8}$, Alexei V. Filippenko ${ }^{8}$, and Nicole Miller ${ }^{3}$
}

\begin{abstract}
An analysis of the first set of low-redshift $(z<0.08)$ Type Ia supernovae monitored by the Carnegie Supernova Project between 2004 and 2006 is presented. The data consist of well-sampled, high-precision optical (ugriBV) and near-infrared (NIR; YJHK $K_{s}$ ) light curves in a well-understood photometric system. Methods are described for deriving light-curve parameters, and for building template light curves which are used to fit Type Ia supernova data in the ugriBVYJH bands. The intrinsic colors at maximum light are calibrated using a subsample of supernovae assumed to have suffered little or no reddening, enabling color excesses to be estimated for the full sample. The opticalNIR color excesses allow the properties of the reddening law in the host galaxies to be studied. A low average value of the total-to-selective absorption coefficient, $R_{V} \approx 1.7$, is derived when using the entire sample of supernovae. However, when the two highly reddened supernovae (SN 2005A and SN 2006X) in the sample are excluded, a value $R_{V} \approx 3.2$ is obtained, similar to the standard value for the Galaxy. The red colors of these two events are well matched by a model where multiple scattering of photons by circumstellar dust steepens the effective extinction law. The absolute peak magnitudes of the supernovae are studied in all bands using a two-parameter linear fit to the decline
\end{abstract}

\footnotetext{
${ }^{1}$ This paper includes data gathered with the $6.5 \mathrm{~m}$ Magellan Telescopes located at Las Campanas Observatory, Chile.

${ }^{2}$ Las Campanas Observatory, Carnegie Observatories, Casilla 601, La Serena, Chile.

${ }^{3}$ Universidad de Chile, Departamento de Astronomía, Casilla 36-D, Santiago, Chile.

${ }^{4}$ Observatories of the Carnegie Institution of Washington, 813 Santa Barbara St., Pasadena, CA 91101.

${ }^{5}$ Texas A\&M University, Physics Department, College Station, TX 77843-4242.

${ }^{6}$ Mitchell Institute for Fundamental Physics and Astronomy.

${ }^{7}$ Infrared Processing and Analysis Center, Caltech/Jet Propulsion Laboratory, Pasadena, CA 91125.

${ }^{8}$ Department of Astronomy, University of California, Berkeley, CA 94720-3411.

${ }^{9}$ Dark Cosmology Centre, Niels Bohr Institute, University of Copenhagen, Juliane Maries Vej 30, 2100 Copenhagen $\varnothing$, Denmark.
} 
rates and the colors at maximum light, or alternatively, the color excesses. In both cases, similar results are obtained with dispersions in absolute magnitude of 0.12-0.16 mag, depending on the specific filter-color combination. In contrast to the results obtained from the comparison of the color excesses, these fits of absolute magnitude give $R_{V} \approx 1-$ 2 when the dispersion is minimized, even when the two highly reddened supernovae are excluded. This discrepancy suggests that, beyond the "normal" interstellar reddening produced in the host galaxies, there is an intrinsic dispersion in the colors of Type Ia supernovae which is correlated with luminosity but independent of the decline rate. Finally, a Hubble diagram for the best-observed subsample of supernovae is produced by combining the results of the fits of absolute magnitude versus decline rate and color excess for each filter. The resulting scatter of $0.12 \mathrm{mag}$ appears to be limited by the peculiar velocities of the host galaxies as evidenced by the strong correlation between the distance-modulus residuals observed in the individual filters. The implication is that the actual precision of Type Ia supernovae distances is $3-4 \%$.

Subject headings: distance scale - dust, extinction - galaxies: distances and redshifts galaxies: ISM - supernovae: general

\section{INTRODUCTION}

The discovery in the late-1990s from measurements of distant Type Ia supernovae (SNe Ia) that the expansion of the Universe is currently accelerating (Riess et al. 1998; Perlmutter et al. 1999) has given rise to an exciting new era in cosmology. The observations appear to require a previously unrecognized form of energy density ("dark energy") which today is the dominant constituent of the Universe. In recent years, observations of SNe Ia to increasingly higher redshifts have confirmed this finding (e.g., Riess et al. 2007; Astier et al. 2006; Wood-Vasey et al. 2007). Measurements of the angular power spectrum of the cosmic microwave background radiation provide independent confirmation, favoring a flat geometry for the Universe with a dominant $73 \%$ of the energy density in the form of dark energy (e.g., see Spergel et al. 2007). Baryon acoustic oscillations (Eisenstein et al. 2005) and measurements of the ratio of X-ray-emitting gas to total mass in galaxy clusters (Allen et al. 2004, 2007) have also provided convincing confirmation of this picture.

Effort is currently focused on determining the nature of dark energy by measuring an equationof-state parameter of the form $w=P /\left(\rho c^{2}\right)$, and its time derivative $\dot{w}$. Several ongoing and future projects aim at filling the Hubble diagram with high-redshift $(z>0.1)$ SNe Ia in order to measure $w$ and $\dot{w}$ with sufficient precision to distinguish among the various proposed models of dark energy. However, these measurements require an improved reference set of SN Ia observations at low redshift. Surprisingly, the currently available low-redshift datasets are neither sufficiently numerous nor homogeneous to adequately complement the high-redshift data.

The Carnegie Supernova Project (CSP) is one of several ongoing efforts to improve the quality 
of the low-redshift data 9 Over the five-year period ending in May 2009, the CSP has obtained densely sampled optical and near-infrared (NIR) light curves of $\sim 100 \mathrm{SNe}$ Ia in a well-understood, homogeneous photometric system (Hamuy et al. 2006, hereafter H06). The NIR was included specifically to address the unknown intrinsic colors and interstellar reddening to SNe Ia. This work presents an analysis of the first release of SNe Ia by the CSP.

In $\S 2$, we present an analysis of the light curves, both optical and NIR, and introduce the methods used to derive parameters which serve to determine distances. In $\S[$, the non-trivial issue of measuring extinction is considered, and the nature of the reddening law in the host galaxies is studied. In $\S$, the properties of SNe Ia as standardizable candles is examined by using Hubbleflow distances to fit the relationship between absolute peak magnitudes, decline rates, and colors or reddenings for different bands. Finally, in $\S$, the implications of our findings are discussed.

This paper is the second of three companion papers presenting first results from CSP observations of low- and high-redshift SNe Ia. The low-redshift data used in the present analysis are described in detail in the first paper by Contreras et al. (2009, hereafter C09). In the third paper, NIR light curves of 35 high-redshift SNe Ia observed with the Magellan Baade 6.5-m telescope are combined with the low-redshift data in order to construct the first rest-frame $i$-band Hubble diagram of SNe Ia (Freedman et al. 2009).

\section{PROPERTIES OF THE LIGHT CURVES}

The CSP carries out its follow-up observations with the Las Campanas Observatory (LCO) Swope 1-m telescope using optical ugriBV bands, and NIR $Y J H$ bands. Additionally, $Y J H K_{s}$ photometry is obtained with the Wide-field Infrared Camera (WIRC) at the du Pont $2.5 \mathrm{~m}$ telescope of LCO. As mentioned in H06, the NIR monitoring of the CSP during the first year of observations was generally not as thorough as that for the optical due to the fact that RetroCam, the NIR camera at the Swope telescope, was not available until September 2005. This situation was much improved during the final four campaigns of the project.

The photometry of each SN is performed relative to nearby field stars which are, in turn, calibrated from observations of standard stars in the Landolt (1992) (BV), Smith et al. (2002) (ugri), and Persson et al. (2004) $\left(Y J H K_{s}\right)$ systems during several photometric nights. Pointspread function (PSF) photometry of the SN is measured after the underlying light from the host galaxy has been subtracted from every follow-up image. Details of the measurement procedures and photometric system are given by H06. Magnitudes are expressed in the natural photometric system of the Swope+CSP bands, as explained in C09. In accordance with that paper, in the remainder of this work we will refer to our optical natural photometry as ugriBV. The final photometry for

\footnotetext{
${ }^{9}$ See Contreras et al. (2009) for a summary of other groups that are producing large databases of low-redshift SN Ia light curves.
} 
the SNe is published by C09.

In the following, the methods employed for deriving light-curve parameters for the SN sample are presented, including the construction of template light curves for the ugriBVYJH bands.

\subsection{Direct Measurements}

The SNe Ia included in this analysis are listed in Table 1. Twenty-six of these SNe have optical observations beginning before $B$-band maximum light and with a cadence of a few days or less, which allows direct functional fits to their data. A subsample of nine SNe in this group also have enough $Y J H$ coverage to allow the same type of fits around maximum light. These fits provide direct measurements of light-curve parameters such as peak magnitude $m^{\max }$, time of maximum $t^{\max }$, and decline rate $\Delta m_{15}(B)$, the latter measured from the $B$-band light curves (Phillips 1993) 10 We therefore decided to directly measure the light-curve shape parameter, $\Delta m_{15}(B)$, whenever possible rather than derive it from template fits in order to tie our luminosity corrections to a purely empirical parameter. These resulting parameters are given in Table 2, labeled as "Spline" in column 7. The fits also serve to build a set of template ugriBVYJH light curves which may be used to fit more poorly observed SNe, as described in $\S 2.2$,

Spline functions are preferred for these direct fits since they are more readily adaptable to the shapes occurring in SN Ia light curves than are other functions such as polynomials. We use the FITPACK library (Dierckx 1993), which employs a variable number of knots depending on the sampling and precision of the data. These spline functions allow the whole time span of the observations to be fit without introducing undesirable oscillations where the coverage is less dense. The high quality of the photometry and the good time sampling of the observations ensures the accuracy of the fits. Errors in the parameters derived from the spline fits are modeled via a Monte Carlo calculation performed by randomly perturbing each data point according to its quoted photometric error and then measuring the scatter of the output measurements.

As mentioned by Contreras et al. (2009), one can expect a certain degree of correlation among light-curve points introduced by the host-galaxy subtraction process because the same template image is used to subtract all of the follow-up images. Not taking the correlation into account may lead to underestimating the derived uncertainties of the light-curve parameters. The effect is expected to be more noticeable when the host-galaxy subtraction is more uncertain, and the photometric precision and coverage are larger. We have estimated this effect by computing the covariance matrix for the data points and including this in the Monte Carlo calculations. The covariance matrix is estimated by examining the photometry of artificial stars which are added to the subtracted images at fixed locations around the SN and which are set to have the same flux

\footnotetext{
${ }^{10}$ Throughout this paper we use the superscript or subscript "max" to denote the quantity measured at the time of maximum light in the corresponding band.
} 
as the $\mathrm{SN}$ at each epoch. In general, these experiments have yielded results in agreement with the case in which the correlations are neglected. Only in a few cases have the uncertainties in the peak magnitudes increased by more than a few thousandths of a magnitude. Therefore, we consider this effect to be negligible for the current analysis.

K-corrections are applied to the observed magnitudes to convert these to the rest frame. For this purpose, we utilize a library of SN Ia template spectra provided by Hsiao et al. (2007) in the optical, and Hsiao et al. (2009) in the NIR. The K-corrections are computed simultaneously with the light-curve fits. The template spectra corresponding to the epochs of the observations are first warped by a smooth function of wavelength in order to make the synthetic colors match the observed ones for all available bands. Since the template spectra cover a limited range of epochs from -15 to +85 days relative to $B$-band maximum, observations outside these limits are ignored.

The accuracy of the K-corrections can be tested by comparing them with values computed from the 194 follow-up spectra obtained by the CSP of the sample SNe. The spectra typically cover the optical griBV bands and a range of epochs from -12 to over +100 days with respect to $B$-band maximum light. In most cases, the agreement with the K-corrections derived from the template spectra is better than \pm 0.02 mag. Unfortunately, the lack of spectral coverage in the $u$ and NIR bands prevents this test from being performed in those bands.

Figure 1 shows the spline fits for all SNe with pre-maximum data in each band. It should be noted that since normal SNe Ia peak earlier in $u$ and $i$ than they peak in $B$ (see $\S 2.4$ and Figure 44), some SNe were not observed before maximum light in these two bands, reducing the sample from 26 in $B$, to 21 and 19 (respectively) in $u$ and $i$.

The value of $\Delta m_{15}(B)$ obtained for each $\mathrm{SN}$ is indicated next to the corresponding curves in Figure 1. The range of decline rates covered by the sample is $0.7<\Delta m_{15}(B)<1.9$ for the $B V g r$ bands, and $0.8<\Delta m_{15}(B)<1.9$ for the $u i Y J H$ bands, which corresponds well to the full range of observed values of $\Delta m_{15}(B)$ for SNe Ia.

The optical data for these $26 \mathrm{SNe}$ start between -12 and -1 days relative to $B$-band maximum light, and end between 17 and over 100 days past maximum, typically extending more than one month after maximum light. The $Y J H$ data for the nine SNe with pre-maximum coverage start between -10 and -1 days, and end between 5 and 90 days past maximum.

Following the work of Hamuv et al. (1996b) and Elias-Rosa et al. (2008), we searched for alternative characterizations of the SN Ia decline rates by comparing $\Delta m_{15}(B)$ with values of $\Delta m_{t}(X)$ for different bands $X$, and times $t$, since maximum. Figure 2 shows the cases for ugriBV which provide the strongest correlations with $\Delta m_{15}(B)$; Figure 3 shows the same results for the NIR $Y J H$ bands. In general, at longer wavelengths, the best correlations with $\Delta m_{15}(B)$ are found at later epochs. Even in the case of the NIR $i Y J H$ bands, where the double-peaked shape of the light curves complicates matters, reasonable correlations with $\Delta m_{15}(B)$ are obtained at $t \sim 35-40$ days - i.e., during the radiative decline. 
The behavior of the timing of maximum light for different bands with respect to $B$ band was also studied. This is shown in Figure 4. For $u g V r$, the delay (or advance for $u$ ) is roughly constant for $\mathrm{SNe}$ of all decline rates. In the $i Y J H$ bands, there is a nearly constant advance of $2-4$ days for SNe with $\Delta m_{15}(B)<1.5$, whereas there is a delay of several days for SNe with $\Delta m_{15}(B) \approx 1.8$. Note that the scatter in Figure 4 is generally greater than the error bars, implying that there is an intrinsic component.

\subsection{Template Light-Curve Fits}

The availability of such a complete set of ugriBVYJH light curves with pre-maximum coverage allows template light curves to be built which can be used to derive light-curve parameters for $\mathrm{SNe}$ with inadequate coverage around maximum brightness. For this purpose, we used the SNOOPy (SuperNovae in Object-Oriented Python) package (Burns et al. 2009), which is derived from the the multi-filter light-curve fitting method developed by Prieto et al. (2006), with a few modifications. The technique for generating templates is briefly summarized below, and in more detail by Burns et al. (2009).

The SNe that were fit with spline functions (see $\S 2.1$ ) form the training dataset. The time of $B$-band maximum light $t^{\max }(B)$, the decline rate measured as $\Delta m_{15}(B)$, and the peak magnitude $m_{X}^{\max }$ in each band $X$ are used to place the photometric data points of the training set in a threedimensional (3-D) space for each band defined by the following variables:

1. The rest-frame epoch with respect to $B$-band maximum, $\Delta t=\left[t-t^{\max }(B)\right] /(1+z)$, where $t$ is the time of the observation and $z$ is the heliocentric redshift of the $\mathrm{SN}$;

2. the decline-rate parameter, $\Delta m_{15}(B)$, considered as a single parameter to characterize all bands; and

3. the K-corrected magnitude relative to maximum light, $\Delta m=m_{X}(t)-m_{X}^{\max }-K_{X}(t)$, for each observed magnitude $m_{X}(t)$.

SN 2006X was removed from the training dataset in ugriBV because of its peculiar behavior, especially in the $B$ band, which showed an abnormally flat decline rate beginning one month after maximum light. This morphology has recently been explained as a light echo produced by dust surrounding this heavily reddened SN (Wang et al. 2008b). However, since there is no evidence that the NIR light curves of SN 2006X suffered such contamination, the data for this event were maintained in the training set for building $Y J H$ templates.

The data points of the training set define a surface in the 3 -D space $\left(\Delta t, \Delta m_{15}(B), \Delta m\right)$ for each band. To produce a smooth interpolation to any point in the 3 -D space, we use a 2 -D variation of the "gloess" algorithm, which is a Gaussian-windowed and error-weighted extension of data-smoothing methods outlined by Cleveland (1979) that was first implemented by 
Persson et al. (2004) for fitting Cepheid light curves. This is the main difference between the method of Prieto et al. (2006) and that of the SNOOPy package (Burns et al. 2009). While the former method uses the fit spline functions as templates and interpolates among them, SNOOPy performs an interpolation among the data points of the SNe. The gloess algorithm ensures a smooth interpolation even with heterogeneously sampled data.

The SNOOPy package generates templates for a given value of $\Delta m_{15}(B)$ by making a slice through the $\left(\Delta t, \Delta m_{15}(B), \Delta m\right)$ surface, interpolating along a constant $\Delta m_{15}(B)$ line. The best-fit time of maximum light $t^{\text {max }}$, decline rate $\Delta m_{15}(B)$, and peak magnitudes $m^{\text {max }}$ are then determined by fitting these templates to the ugriBVYJH data of each SN via $\chi^{2}$ minimization, with K-corrections computed in an iterative fashion, as described in $\S 2.1$. Uncertainties in the fit parameters are taken from the diagonals of the covariance matrix output from the LevenbertMarquardt least-square routine used to fit the templates (Burns et al. 2009). Additionally, we have tested the possible effect of correlated errors in the photometry due to the use of a single hostgalaxy image in the subtraction process (see Contreras et al. 2009). Similarly to the results of the tests for spline fits that are given in $\S$ 2.1, in the case of template fits we find that the effect is very small in all but a few examples. We have thus decided to use the uncertainties that are derived from uncorrelated errors.

This way, it is possible to derive the light-curve parameters for each SN, including the objects in our sample which were not observed until after maximum light. Table 2 lists the results of these template fits for the cases in which a spline function could not be fit; these are labeled as "Templ." in column 7. The template fits themselves are shown by C09 together with the observed light curves for all SNe in the sample.

In order to test the accuracy of the method, template fits were performed for the subset of wellobserved SNe and the resulting parameters were compared with those obtained directly from spline fits ( $\S 2.1$ ). These comparisons are shown in Figure 5 for the peak magnitudes in ugriBVYJH, and for the decline-rate parameter $\Delta m_{15}(B)$. The average difference in peak magnitudes is $\pm 0.05 \mathrm{mag}$ or less for all of the optical filters except the $i$ band, with no obvious systematic differences except, perhaps, in $u$. The dispersion in the $i Y J H$ bands is $\sim \pm 0.1 \mathrm{mag}$, with some evidence for systematic differences in $Y J H$. A similar situation holds for the differences in the time of maximum as measured with spline and template fits, where good consistency $( \pm 0.6$ days or less) is found in ugr $B V$, but the $i Y J H$ bands yield worse agreement. The relatively poor precision of the $Y J H$ template fits is at least partly due to the small sample used to derive the templates. However, the large dispersion in $i$ is the product of variations in the morphology of the secondary maximum (see $\S$ 2.3). Figure 5 shows that, except at the fastest decline rates, there is reasonable consistency between the decline rate as measured by spline and template fits.

The uncertainties associated with extrapolating the templates to obtain peak magnitudes and decline rates for those SNe caught after maximum light are more difficult to quantify. Clearly, the later the observations begin, the larger are the possible extrapolation errors. One way to approach 
this issue is to take the spline fits of the light curves of the SNe in the sample that were wellobserved before and after maximum, resample these to simulate a SN whose observations did not begin until after maximum, and then fit these data using the ugriBVYJH templates to recover the time of $B$ maximum, $\Delta m_{15}(B)$, and the maximum-light magnitudes. Calculations of this type will be presented in more detail in a future paper (Burns et al. 2009), but for the representative case of a SN for which coverage began $\sim 1$ week after maximum and continued until $\sim 70$ days after maximum, we find a typical random uncertainty of $\sim 0.1$ mag in the peak magnitude, averaged over all filters, and a much smaller systematic difference of $\sim 0.03$ mag. Experience shows that for SNe with photometry beginning later than this, template fits are not reliable. Fortunately, only one event in the Contreras et al. (2009) sample, SN 2004dt, falls into this category; hence, it is excluded from the analysis in the present paper.

The lack of a sufficiently large set of SNe with good coverage around maximum light in the $K_{s}$ band prevents the production of template light curves as was done for all other bands. Instead, we use the polynomial template light curve introduced by Krisciunas et al. (2004a). A stretch factor (Perlmutter et al. 1997) based on the decline rate of each SN is applied to the time axis of the polynomial - along with the redshift-dependent time dilation - which is then fit to the data in order to obtain a peak magnitude. This kind of fit may be utilized for SNe with data in the range of validity of the template, which is between -12 and +10 days relative to $t^{\max }(B)$ for a $\mathrm{SN}$ of stretch factor $S=1$. The specific formula employed to convert between $\Delta m_{15}(B)$ and $S$ is that given by Jha et al. (2006):

$$
S=\frac{3.06-\Delta m_{15}(B)}{2.04}
$$

\subsection{The Secondary Maximum}

Höflich et al. (1995) and Pinto \& Eastman (2000) were the first to attempt a theoretical understanding of the NIR secondary maximum in SNe Ia. More recently, Kasen (2006) modeled the NIR light curves of SNe Ia, arguing that the timing and strength of the secondary maximum is a consequence of the ionization evolution of the iron-peak elements in the ejecta. A specific prediction is that more luminous SNe should have a later and more prominent secondary maximum. In order to test this, we examined the delay and relative strength of the secondary maximum in the $i$-band

light curves of the CSP SNe with the best coverage. Using the nomenclature of Kasen (2006), the following parameters were measured:

1. The phase of the peak of the secondary maximum with respect to $B$-band maximum, $t_{2}-t_{B}$;

2. the difference in magnitudes between the secondary maximum and the local minimum between the primary and the secondary maxima, $m_{2}-m_{0}$; and

3. the difference in magnitudes between the primary and secondary maxima, $m_{1}-m_{2}$. 
Figure 6 shows the results plotted as a function of $\Delta m_{15}(B)$. If the decline-rate parameter $\Delta m_{15}(B)$ (or peak luminosity) is associated with the amount of ${ }^{56} \mathrm{Ni}$ mass synthesized in the ejecta (Arnett 1982; Arnett et al. 1985; Nugent et al. 1995; Stritzinger et al. 2006), then Figure 6 can be compared directly with Figure 11 of Kasen (2006). Indeed, the upper panel of Figure 6 shows a strong correlation between $\Delta m_{15}(B)$ and the timing of the secondary maximum, confirming previous results by Hamuy et al. (1996b) and Elias-Rosa et al. (2008). However, the middle and lower panels of Figure 6 show that there is little evidence for a dependence between $\Delta m_{15}(B)$ and the strength of the secondary maximum, as measured with respect to either the primary maximum or the local minimum between the two maxima. Kasen (2006) points out that both the timing and strength of the secondary maximum can also be affected by the outward mixing of ${ }^{56} \mathrm{Ni}$ in the ejecta, the amount of stable iron group elements produced in the explosion, the progenitor metallicity, and the abundance of calcium in the ejecta. Of these, mixing appears to have the greatest effect on the strength of the secondary maximum, and therefore may be responsible for the lack of obvious correlations in the middle and bottom panels of Figure 6 .

Krisciunas et al. (2001) suggested a different way of measuring the strength of the $I$-band second maximum which consists of converting the light-curve observations to fluxes normalized to the flux at the primary maximum, fitting a high-order polynomial to these, and then determining the mean flux (based on an integration of the polynomial) from 20 to 40 days after the time of $B$ maximum. They found a good correlation between this parameter, $\langle I\rangle_{20-40}$, and the decline rate. Similar measurements were carried out for the $i$-band light curves of the best-observed subsample of CSP SNe, and plotted vs. $\Delta m_{15}(B)$ in Figure 7 . Illustrated as a solid line are the same measurements for the family of SNOOPy templates. There is a clear trend in this diagram in the sense that luminous, slowly declining SNe generally display stronger secondary maxima. However, there is also a significant and real dispersion which is illustrated by the four labelled SNe - 2006D, 2006bh, 2004ef, and 2004eo. The left half of Figure 8 shows that all four have very similar $B$ and $V$ light curves covering a narrow range of decline rates $\left(\Delta m_{15}(B)=1.37-1.42\right)$. Nevertheless, there are significant differences in the strength and morphology of the $i$-band secondary maximum which, again, may reflect varying amounts of mixing of the ${ }^{56} \mathrm{Ni}$ into the ejecta (Kasen 2006).

These variations in the strength of the secondary maximum represent a significant problem for template fitting in the $i Y J H K$ bands, where the strength of the secondary maximum is assumed to be a smoothly varying function of $\Delta m_{15}(B)$. This is illustrated in the right half of Figure 8 , where the difference in the maximum-light magnitudes in $B V i$ as derived from template and spline fits is plotted for the same four SNe (2006D, 2006bh, 2004ef, and 2004eo) with nearly identical decline rates. In the $B$ band, the template fits give results that are in excellent agreement $( \pm 0.02 \mathrm{mag})$ with the direct measurements from the spline fits. The agreement in $V$ is nearly as good, but in the $i$ band, the template fits give errors as large as $\sim \pm 0.15$ mag. Figure 8 shows that these errors are a function of the strength of the secondary maximum: template fits to SNe with a weak secondary maximum will yield maximum-light magnitudes that are too faint, whereas fits to $\mathrm{SNe}$ with a strong secondary maximum will give maximum-light magnitudes that are too bright. This 
effect most likely explains the relatively large dispersion in the difference in the peak $i$ magnitudes measured from template and spline fits observed in Figure 5, Until an observational parameter can be found which makes these differences predictable, they will remain a significant impediment to the fitting of template light curves in the $i Y J H K$ bands. Quantities such as $\langle I\rangle_{20-40}$ or $m_{2}-m_{0}$ may prove to be such a parameter, but a larger sample of SNe is needed to test their utility.

\subsection{Light-Curve Parameters}

The results of the light-curve fits for the SNe in our sample are summarized in Table 2, where the times of maximum $\left(t^{\max }\right)$ and apparent peak magnitudes $\left(m^{\max }\right)$ are listed for all bands. Also given are decline-rate parameters $\left(\Delta m_{t}\right)$ for each filter expressed as the amount in magnitudes that the light curve declines between maximum light and an epoch $t$ days after maximum. Finally, the number of data points and range of epochs covered with respect to the time of maximum, as well as the fitting method (spline functions, $\S$ 2.1, or templates, $\S$ 2.2.), are specified for each band.

Based on the quality of the data and fits, we define a subsample of SNe with the best-measured light-curve parameters, mostly via direct fits but also through template fitting. Twenty-nine SNe belong to this group of "best-observed" events. These objects are identified in Table 1, in Table 2 , their names are set in bold face. Special use will be made of this subsample when studying the relation between peak luminosity and decline rates in $\S$.

\section{HOST-GALAXY REDDENING}

The $E(B-V)_{\text {Gal }}$ values listed in Table 1 derived by Schlegel, Finkbeiner, \& Davis (1998) from dust maps of the Galaxy may be used to correct for the interstellar reddening produced in our Galaxy. Beyond the Milky Way, the situation is considerably more complicated as there are at least three separate potential sources of reddening due to (a) dust in the intergalactic medium, (b) dust in the interstellar medium of the host galaxy, and (c) dust associated with the circumstellar material of the SN progenitor. We have no a priori knowledge of the dust properties in these different environments and the reddening laws could, in principle, be different for all three. Nevertheless, by comparing the colors of SNe with similar decline rates and taking advantage of the wide wavelength coverage of our observations, one can hope to discern, in general terms, the combined effect of these different sources of reddening, which we lump together and refer to as the "host-galaxy reddening."

\subsection{Low-Reddening Sample}

In order to use color information to determine reddening of the SN light produced in the host

galaxy, we follow the procedure of Phillips et al. (1999) of selecting a subsample of SNe which 
are suspected to have suffered little or no dust extinction. This subsample will be used to derive intrinsic colors for SNe Ia as a function of decline rate which, in turn, will serve as a reference for computing color excesses for the whole SN sample.

The criteria employed to select the low-reddening subsample of SNe are (a) SNe which occurred in E/S0-type galaxies, or SNe located away from the arms or nuclei of spiral galaxies, and (b) absence of detectable interstellar Na I D lines in early-time spectra.

Ten SNe from the sample fulfill both criteria: SNe 2004eo, 2005M, 2005al, 2005am, 2005el, 2005hc, 2005iq, 2005ke, 2005ki, and 2006bh. In what follows, these objects are used to derive intrinsic color relations as a function of the decline-rate parameter $\Delta m_{15}(B)$. We use colors corrected for Galactic reddening since such correction is, in most cases, small (see Table 10) and known to higher accuracy than the host-galaxy reddening. We thus do not perform any cut based on Galactic reddening. We assume a value of $R_{V}=3.1$, which may not be exactly correct for each SN. However, since the maximum Galactic reddening for this sample of ten $\mathrm{SNe}$ is $E(B-V)=0.14 \mathrm{mag}$, uncertainties in this correction will be small.

\subsection{The $B-V$ Tail}

The subsample of low-reddening SNe can be used to study the behavior of the $B-V$ color approximately one month after maximum light. It was first found by Lira (1995) that unreddened SNe Ia follow a very similar linear regime in $B-V$ between one and three months after maximum. As shown in Figure 9, we confirm this result and derive the following intrinsic color law for the "tail" of the $B-V$ color evolution:

$$
(B-V)_{0}=0.732(0.006)-0.0095(0.0005)\left(t_{V}-55\right) .
$$

For consistency with Phillips et al. (1999), rest-frame days since maximum $V$ light, $t_{V}$, is used for the time axis. Equation (2) is valid over the range $30<t_{V}<80$ days and yields a dispersion of $0.077 \mathrm{mag}$. The SNe employed in the fit cover a broad range of decline rates, $0.85<\Delta m_{15}(B)<$ $1.76 \mathrm{mag}$. This relation is similar to the one of Phillips et al. (1999), who found, for a sample of six SNe and a different photometric system, $(B-V)_{0}=0.784-0.0118\left(t_{V}-55\right)$ with a dispersion of 0.06 mag. Figure 9 shows that the residuals for individual $\mathrm{SNe}$ are strongly correlated, which implies that some of the $10 \mathrm{SNe}$ in this subsample actually do suffer measurable host-galaxy reddening, or that there is an intrinsic dispersion in the Lira relationship, or both. We also note that the three SNe in our sample which occurred in early-type galaxies (E/S0), namely SNe 2005al, 2005el, and 2005ki, show remarkably similar $B-V$ color evolution - and also in other optical colors - during the tail, with an average deviation of $-0.05 \pm 0.003 \mathrm{mag}$ from equation (2), and a dispersion of $\sim 0.035$ mag.

Equation (2) can be used to estimate color excesses, $E(B-V)_{\text {tail }}$, for the SNe in our sample with 
observations in the tail 11 The results are listed in column (2) of Table 4. The uncertainties in these color excesses reflect only the photometric errors in the data points. In considering the color excess for any particular SN, the observed dispersion of 0.077 mag in the Lira law should also be added in quadrature to these errors. However, in the analysis presented in $\S 4.2$ of the precision to which SNe Ia may be used as standardizable candles, this dispersion is included in the $\sigma_{\mathrm{SN}}$ values that are yielded by the fits, and the errors used for the color excesses are those given in Table 4. Note that since equation (2) was derived by fitting to the average color evolution of the low-reddening sample, it is guaranteed to generate some small negative color excesses. Phillips et al. (1999) dealt with this by applying a Bayesian prior consisting of a one-sided Gaussian distribution of $A_{B}$ values with a maximum at zero and $\sigma=0.3 \mathrm{mag}$. More recently, Jha, Riess, \& Kirshner (2007) used a prior consisting of a Gaussian with $\sigma_{(B-V)}=0.068 \mathrm{mag}$, representing the intrinsic dispersion in color, convolved with an exponential reddening distribution with a scale length $\tau_{E(B-V)}=0.138 \mathrm{mag}$ which they derived from fits to nearby SN Ia light curves by assuming a particular intrinsic color distribution. However, until it is possible to confidently separate dust reddening from intrinsic color variation, we choose not to apply a prior to the color-excess measurements.

For each SN, we tested the agreement of the $B-V$ tail slope with that of equation (2). Good consistency was generally found, with the notable exception of SN 2006X, the most highly reddened $\mathrm{SN}$ in the sample. As mentioned in $\S 2.1$, this SN showed a peculiar behavior in the $B$ band at the epochs considered here, probably due to the appearance of a light echo (Wang et al. 2008b).

Figure 10 displays a plot of $E(B-V)_{\text {tail }}$ versus the equivalent width of the absorption in the Na I D lines produced in the host galaxies as measured from spectra we obtained of the SNe. Generally speaking, these spectra are of low wavelength resolution, and the Na I D lines are only visible when the equivalent width reaches $\sim 1 \AA$ or more. The red symbols with dashed error bars in Figure 10 indicate the low-reddening sample. Four of the eight SNe with the largest color excesses show detectable $\mathrm{Na}$ I D absorption, and the two with the largest color excesses, 2004gu and 2005lu, correspond to the two with the highest Na I D equivalent widths. However, two SNe with slightly negative values of $E(B-V)_{\text {tail }}$, SNe 2005bg and $2006 \mathrm{mr}$, also show strong Na I D absorption. In the case of SN 2005bg, the estimate of $E(B-V)$ tail is based on a single $B-V$ color measurement in the tail, and therefore may be unreliable. SN $2006 \mathrm{mr}$ was a very rapidly declining event which occurred in the dusty central regions of Fornax A (NGC 1316). Garnavich et al. (2004) studied a small sample of fast-declining SNe Ia and found that they followed the Lira relationship fairly well, but SN 2006mr suggests that there may be exceptions. In any case, the correlation between color excess and the Na I D equivalent width is known to be poor (Blondin et al. 2009).

Figure 10 shows that five additional SNe (2004ey, 2005ag, 2006D, 2006ax, and 2006gt) have color excesses $E(B-V)_{\text {tail }}<0.08 \mathrm{mag}$ and undetectable Na I D absorption. In the following analysis, these five SNe are added to the original subsample of ten assumed to have suffered little

\footnotetext{
${ }^{11}$ We remind the reader that equation (2) and all other relationships given in this paper which involve the CSP observed magnitudes are valid only for photometry in the natural system of the Swope+CSP bands.
} 
or no host-galaxy reddening.

\subsection{Pseudocolors at Maximum Light}

Since the CSP data - and generally most SN Ia observations - have better coverage around maximum light than during the declining tail, it is important to develop methods to determine color excesses from observations around the time of maximum. Using the peak magnitudes (corrected for Galactic reddening and the K-correction) derived in $\S 2$, pseudocolor 12 can be computed as the differences between the maximum-light magnitudes in two given bands. The dependence of these pseudocolors on the decline rate, parameterized by $\Delta m_{15}(B)$, can then be investigated for a subsample of SNe with low reddening.

Figure 11 shows the pseudocolors as a function of $\Delta m_{15}(B)$ for several band combinations which will be used in the analysis of $\S$ 4. The solid points in the figure correspond to the $15 \mathrm{SNe}$ with low reddening. As expected, in all colors these SNe lie at the lowest values, following a roughly linear trend with decline rate over the range $0.8<\Delta m_{15}(B)<1.7$ mag. At the fastest decline rates $\left(\Delta m_{15}(B)>1.7 \mathrm{mag}\right)$, however, the pseudocolors at maximum light are significantly redder than these linear trends would predict. Table 3 summarizes straight-line fits to the colors of the low-reddening SNe with decline rates $0.8<\Delta m_{15}(B)<1.7 \mathrm{mag}$; these fits are plotted as dashed lines in Figure 11. Note that the slopes of the relations are largest when the $u Y J H$ bands are involved. In general, there is a correlation between the dispersion in color and the difference in the effective wavelengths, $\Delta \lambda$, of the two filters that form the color. Within this trend, the colors involving the $u$ filter have a relatively higher dispersion for their $\Delta \lambda$ values, whereas the colors involving the $J$ filter have a lower dispersion.

In the case of the $\left(B^{\max }-V^{\max }\right)$ pseudocolor, the following linear law for the $14 \mathrm{SNe}$ with low reddening and $0.8<\Delta m_{15}(B)<1.7$ mag is found:

$$
\left(B^{\max }-V^{\max }\right)_{0}=-0.016(0.014)+0.12(0.05)\left[\Delta m_{15}(B)-1.1\right] .
$$

The root-mean square ( $\mathrm{rms})$ of the fit is $0.06 \mathrm{mag}$. This relation is slightly redder than that given by Phillips et al. (1999) and Altavilla et al. (2004). We use this intrinsic-color law to derive color excesses $E(B-V)_{\max }$ for all $\mathrm{SNe}$ in the range $0.7<\Delta m_{15}(B)<1.7 \mathrm{mag}$, computed as $\left(B^{\max }-\right.$ $\left.V^{\max }\right)-\left(B^{\max }-V^{\max }\right)_{0}$. The resulting values are listed in column 3 of Table 4 . The uncertainties in these color excesses are calculated from the photometric errors in the peak magnitudes.

The top-left panel of Figure 12 shows a comparison of the $E(B-V)_{\max }$ measurements with

\footnotetext{
${ }^{12}$ The term pseudocolor is employed to stress the fact that these quantities do not represent the actual color of the $\mathrm{SNe}$ at any time but are just the difference between the magnitudes at maximum light of two bands, which occur at different times.
} 
the $E(B-V)_{\text {tail }}$ values from $\oint$ 3.2. The sample of 21 objects consists of the best-observed SNe for which both color excesses could be measured. In addition, the highly reddened SN 2006X has been excluded since it clearly deviates from the behavior of the rest of the SNe in the $B-V$ tail, as mentioned in $\S 3.2$. One can see that there is a small but systematic difference between these two color-excess measurements which, unexpectedly, appears to be correlated with the magnitude of the color excess. A formal fit to the data (plotted as a solid line in Figure 12) gives $E(B-$ $V)_{\text {tail }}-E(B-V)_{\max }=0.032-0.496 E(B-V)_{\max }$, with a dispersion of 0.06 mag. The top-right panel of Figure 12 shows that this difference also seems to correlate with the color of the SN. Since $E(B-V)_{\text {tail }}$ and $E(B-V)_{\max }$ are "observed" color excesses, as opposed to "true" color excesses (see Phillips et al. 1999), and are measured at different epochs in the color evolution of the SN, one would expect there to be a small systematic difference between the two that would correlate with the SN color. This effect is produced primarily because the effective wavelengths of the filters evolve due to the significant color evolution of the SN spectral energy distribution, but there is also a smaller dependence on the total amount of dust reddening itself. The Hsiao et al. (2007) spectral template may be used to calculate the expected magnitude of these combined effects, which is plotted as a dotted line in both of the top panels of Figure 12. The observed systematic differences are greater than can be explained in this way, although the significance is only at the $\sim 2 \sigma$ level.

Since the method of estimating the intrinsic colors at maximum by bootstrapping from the Lira relation was first utilized by Phillips et al. (1999), we have examined the color excesses derived by these authors. Interestingly, the same trends observed in the top two panels of Figure 12 are clearly present in those measurements. Not only does this appear to confirm the reality of the effect, but it also argues that it is not an artifact of the CSP dataset.

The lower-left panel of Figure 12 shows that $E(B-V)_{\text {tail }}-E(B-V)_{\text {max }}$ does not depend significantly on the decline-rate parameter $\Delta m_{15}(B)$. The measurements suggest that there may be a weak correlation with the absolute $B$ magnitude (corrected for decline rate), but this requires confirmation by more data. We do not currently understand the underlying causes of these correlations, but the fact that they exist implies that there is a component of one or the other of these color-excess measurements that is not due to interstellar dust.

Keeping this caveat in mind, the fits corresponding to $\left(V^{\max }-X_{\lambda}^{\max }\right)$ for $X_{\lambda}=u g r i B Y J H K_{s}$ can be used to derive color excesses $E\left(V-X_{\lambda}\right)_{\max }$ for all SNe with decline rates in the range $0.8<\Delta m_{15}(B)<1.7$ mag. Columns 4 through 11 of Table 4 list these color excesses.

\subsection{The Reddening Law}

The availability of photometric data spanning the $u$ through $K_{s}$ bands enables the properties of the host-galaxy reddening to be examined. Assuming that the dust extinction in the host galaxies obeys the law introduced by Cardelli. Clavton. \& Mathis (1989) and modified by O'Donnell (1994) (hereafter referred to as the "CCM+O law"), we can investigate which value of the total-to-selective- 
absorption coefficient $R_{V}$ is favored by SN Ia data. Extinction in the Galaxy is well approximated by the $\mathrm{CCM}+\mathrm{O}$ law with an average value of $R_{V} \approx 3$ (e.g., Fitzpatrick \& Massa 2007).

\subsubsection{Optical-NIR Color Excesses}

An average value of $R_{V}$ for the subsample of best-observed SNe can be derived by comparing the color excesses between the optical and NIR bands presented in $\S 3.3$ with the values of $E(B-V)_{\max }$. The use of optical-NIR color excesses ensures the necessary leverage in wavelength to provide a more precise measurement of the reddening law, even for SNe with low reddening. In Figure 13 , comparisons of $E(V-X)_{\max }$ with $X \equiv i Y J H$ are shown.

Fits to the slopes $E(V-X)_{\max } / E(B-V)_{\max }$ were carried out for all four bands considered here. The slope were then converted to values of $R_{V}$, using equations (A5) and (A6) in Krisciunas et al. (2006) and the CCM+O law coefficients $a_{X}$ and $b_{X}$ derived as explained in Appendix B. Table 5 summarizes the results of these fits. The data favor a low value of $R_{V}$, with an average of $R_{V}=$ $1.69 \pm 0.05$ from the four fits. The corresponding slope for this average is indicated by the solid red lines in Figure 13.

If, however, the two highly reddened SNe, SN 2005A and SN 2006X, are excluded from these fits, the uncertainties increase but the favored values of $R_{V}$ become larger and compatible with the standard $R_{V} \approx 3$. An average of $R_{V}=3.2 \pm 0.4$ is derived from the four fits, and is indicated by the dashed blue lines in Figure 13 .

This is a very interesting result which may indicate that SNe with low or moderate reddening are affected by standard dust extinction, while very highly reddened SNe suffer relatively more reddening than extinction, as inferred from a low value of $R_{V}$. We will return to this finding after analyzing the calibration of SNe Ia as standardizable candles in $\S$ 4,

\subsubsection{Highly Reddened Supernovae}

Highly reddened objects provide the most precise information about the behavior of extinction as a function of wavelength. Our sample includes two objects, SNe 2005A and 2006X, for which $E(B-V) \gtrsim 1.0$ mag. Although the optical light curves of SN $2006 \mathrm{X}$ showed evidence of a light echo, this did not appear until $\sim 1$ month after maximum light (Wang et al. 2008b), and so the peak magnitudes should be uncontaminated. Figure 14 shows the values of $E\left(V-X_{\lambda}\right)$ derived for both $\mathrm{SNe}$ (see $\S(3.3)$ plotted as a function of the effective wavelength of each filter $X$. Note that at these extreme values of the color excesses, it is necessary to convert the observed values to true color excesses, and to also correct $\Delta m_{15}(B)$ for reddening effects (see Phillips et al. 1999). Table 6 gives

the linear relations for converting from observed to true color excesses that are derived using the Hsiao et al. (2007) template spectra. Using the same spectra, the following relation for correcting 
the observed decline rate for reddening is found:

$$
\Delta m_{15}(B)_{\text {true }}=\Delta m_{15}(B)_{\text {observed }}+0.065 E(B-V)_{\text {observed }}
$$

Assuming that the reddening of SNe 2005A and 2006X is produced by dust that is described by the $\mathrm{CCM}+\mathrm{O}$ reddening law, fits to the color excesses in Figure 14 give values of $R_{V}$ of $1.68 \pm 0.10$ and $1.55 \pm 0.07$, respectively. These fits are plotted as solid lines in Figure 14, and are to be compared with the dotted lines which show the best-fitting $\mathrm{CCM}+\mathrm{O}$ reddening law where $R_{V}$ is fixed at its canonical Galactic value of 3.1. Although the CCM+O law does a good job in the optical and NIR, it fails miserably in fitting the $(V-u)$ color excesses. Recently, Goobar (2008) has considered a model where multiple scattering of photons by circumstellar dust steepens the effective extinction law, converting it to a power law. When this model is fit to the color excesses of SNe 2005A and 2006X, excellent agreement at all wavelengths is obtained with Large Magellanic Cloud (LMC) dust and a power-law index $p \approx-2.4$. These fits are plotted as dashed lines in Figure 14. Thus, the data would appear to favor a model where the reddening of these two objects is produced by dust that is local to the SN. In this context, it is interesting to note that short-term variations in the interstellar Na I D absorption lines in the spectrum of SN 2006X were observed by Patat et al. (2007) and interpreted as evidence for the existence of significant circumstellar material.

SN 2006X has also been studied in detail by Wang et al. (2008a), who found a best fit to the data with $R_{V}=1.48 \pm 0.06$, in excellent agreement with our results. However, these authors concluded that the data, including their $U$-band photometry, were matched quite well by the CCM reddening law. The Wang et al. (2008a) color-excess measurements for SN 2006X were derived through comparison with a few individual SNe as well as via the method we employ here of looking at the maximum-light colors as a function of the decline rate. In general, there is good agreement between their measurements and our own except for the critical $U$ band, where Wang et al. (2008a) find $E(U-V)_{\text {true }}=2.65 \pm 0.14$ but we derive $E(u-V)_{\text {true }}=3.11 \pm 0.14$. Some of this discrepancy may be due to the fact that the sample of SNe Ia with well-observed light curves in the $U$ or $u$ bands is still fairly small, although in looking at Figure 11, it is difficult to see how the value of $E(u-V)$ could be overestimated by $0.4 \mathrm{mag}$. Although the $u$ filter response function is the least well-determined of the CSP bands, the color excesses for SNe 2005A and 2006X were derived with respect to other SNe Ia observed with exactly the same filter, and in the same photometric system. Thus, the color excess is well determined. The largest uncertainty is the effective wavelength that is associated with the CSP $u$ band, which we estimate to be $<200 \AA$. This maximum possible error is indicated by the horizontal error bars on the $u$ points in Figure 14. The conclusion is that the uncertainty in the $u$-band response function cannot explain the discrepancy.

Only two other objects in our sample, SNe 2005kc and 2006eq, have significant host-galaxy reddenings and observations in the $Y J H$ bands. Fits to the color excesses of these two SNe with both the $\mathrm{CCM}+\mathrm{O}$ and Goobar (2008) reddening laws are displayed in Figure 15. The observations of SN 2005kc are consistent with either the CCM+O law with $R_{V}=4.4 \pm 0.6$, or a Goobar power 
law with $p=-0.7 \pm 0.2$. In the case of SN 2006eq, the measurements may be fit with either a $\mathrm{CCM}+\mathrm{O}$ law with $R_{V}=1.1 \pm 0.8$, or a Goobar power law with $p=-3.5 \pm 1.1$. Thus, the results for SN 2006eq are similar to those obtained for SNe 2005A and 2006X, but the reddening law for SN 2005kc appears to be quite different, perhaps even consistent with normal Galactic reddening. These results confirm those of Figure 13, where SNe 2005kc and 2006eq are the two points with highest $E(B-V)$ values among the moderately reddened SNe. These two SNe illustrate the power of optical-NIR color excesses in differentiating the reddening law. More well-observed, moderately reddened events are clearly needed to advance further in the study of the reddening laws of SNe Ia, and to look for possible correlations with other properties of the SN or its environment.

\section{TYPE IA SUPERNOVAE AS STANDARDIZABLE CANDLES}

In this section, the homogeneous sample of CSP light curves is used to re-evaluate the precision to which SNe Ia may be used as standardizable candles. Two different approaches for calibrating the absolute magnitudes of SNe Ia are considered. We start with the two-parameter method introduced by Tripp (1998) in which the correlation between absolute peak magnitude, decline rate, and color is modeled for different choices of magnitudes and colors. We then move on to use the color excesses determined in $\S 3.4 .1$ to examine the correlation of reddening-corrected, absolute peak magnitudes versus decline rate, following the approach of Phillips et al. (1999). The former method makes no assumptions about the intrinsic colors of SNe Ia; it simply considers decline rates and colors as two parameters that serve to improve the precision of SNe Ia as distance indicators. This formalism was originally employed by Tripp \& Branch (1999) to derive cosmological parameters from SN Ia data, and is part of the SALT (Guy et al. 2005) method used by Astier et al. (2006). The color term in this type of analysis makes no distinction between differences in intrinsic colors or in the amount of reddening suffered by the SNe. On the other hand, the second approach explicitly assumes that one can derive color excesses by determining the intrinsic colors of SNe Ia, and apply these as extinction corrections to the observed peak magnitudes.

To calculate luminosity distances for the SNe, the following approximation is employed which is valid to a precision of better than $0.25 \%$ at redshifts $z<0.1$ in a two-component (matter + dark energy) model of the Universe:

$$
d_{L}\left(z_{\mathrm{CMB}} ; H_{0}, \Omega_{M}, \Omega_{\Lambda}\right)=\frac{\left(1+z_{\mathrm{helio}}\right)}{\left(1+z_{\mathrm{CMB}}\right)} \frac{c}{H_{0}}\left[z_{\mathrm{CMB}}+\frac{1}{2}\left(\Omega_{\Lambda}-\frac{\Omega_{M}}{2}+1\right) z_{\mathrm{CMB}}^{2}\right]
$$

where $z_{\text {helio }}$ is the heliocentric redshift of the host galaxy, $z_{\mathrm{CMB}}$ is the redshift referred to the CMB rest frame, and the cosmological parameters were set to the standard values $H_{0}=72 \mathrm{~km}$ $\mathrm{s}^{-1} \mathrm{Mpc}^{-1}, \Omega_{M}=0.28$, and $\Omega_{\Lambda}=0.72$ (Spergel et al. 2007). The factor $\left(1+z_{\text {helio }}\right) /\left(1+z_{\mathrm{CMB}}\right)$ accounts for the fact that the photon redshift is observed with respect to the heliocentric reference system. The heliocentric redshifts for the sample SNe are given in Table 1. Also given in this table 
are the redshifts in the $\mathrm{CMB}$ frame calculated from the heliocentric redshifts using the velocity vector determined by Fixsen et al. (1996) from the COBE data (and which are available via the NASA/IPAC Extragalactic Database, NED). The uncertainty in the redshifts due to peculiar velocities is assumed to be $\sigma_{z}=0.001$ (300 $\mathrm{km} \mathrm{s}^{-1}$ in velocity). With the exception of the three SNe listed in Table 7 with $z<0.01$ that were hosted by galaxies with direct distance measurements based either on Cepheids or the surface brightness fluctuation (SBF) method, we restrict the sample to those SNe with redshifts $z>0.01$, where peculiar recession velocities become at least one order of magnitude smaller than the cosmological velocities.

In the analyses presented here, the observed peak magnitudes of Table 2 are corrected for

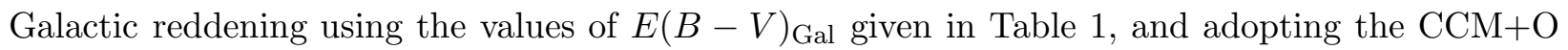
extinction law with $R_{V}^{\mathrm{Gal}}=3.1$.

\subsection{Calibration Using Decline Rates and Colors}

The two-parameter model of Tripp (1998) assumes that the distance modulus of a SN has the following dependence on decline rate and color:

$$
\tilde{\mu}_{X}=m_{X}-M_{X}(0)-b_{X}\left[\Delta m_{15}(B)-1.1\right]-\beta_{X}^{Y Z}\left(m_{Y}-m_{Z}\right),
$$

where $m_{X}$ is the observed peak magnitude in a given band $X$, and $\left(m_{Y}-m_{Z}\right)$ is a pseudocolor at maximum brightness from any choice of bands $Y$ and $Z$. The fit parameters are the peak absolute magnitude of SNe Ia with $\Delta m_{15}(B)=1.1$ and zero color, $M_{X}(0)$; the slope of the luminosity vs. decline-rate relation, $b_{X}$; and the slope of the luminosity-color relation $\beta_{X}^{Y Z}$. Using measurements of peak magnitudes, pseudocolors, and decline rates $\left\{m_{X i} ; \Delta m_{15}(B)_{i} ;\left(m_{Y}-m_{Z}\right)_{i}\right\}(i=1, \ldots, N)$ for a sample of $N \mathrm{SNe}$, the best-fit parameters are solved for via $\chi^{2}$ minimization, as explained in Appendix $\mathrm{A}$. This is equivalent to minimizing the dispersion in the Hubble diagram. Note that a constant term has been added to the measurement uncertainties that appear in the denominator of the $\chi^{2}$ in equation (A4) to account for possible intrinsic dispersion of the SN Ia data about the model given by equation (무).

The Tripp (1998) model has traditionally been applied to fits of $B$ versus $\Delta m_{15}(B)$ and $B-V$. Such an analysis is presented here, along with a fit of $J$ versus $\Delta m_{15}(B)$ and $V-J$.

Table 8 summarizes the results of the fits. Fit 1 was done using the entire sample of $32 \mathrm{SNe}$ with reliable distances and the combination of $B$ versus $\Delta m_{15}(B)$ and $B-V$. This fit is shown in panel (a) of Figure 16, and yields a total dispersion of $0.17 \mathrm{mag}$ and an intrinsic dispersion $\sigma_{\mathrm{SN}}=0.12 \mathrm{mag}$. Performing the fit with the subsample of 26 best-observed SNe (Fit 2) reduces the scatter to $0.15 \mathrm{mag}$ and the derived intrinsic dispersion to just 0.09 mag without modifying substantially the fit parameters. This is shown in panel (b) of Figure 16. 
Note that the fits described above include SNe with all the available decline rates; specifically, they include the fast-declining SNe with $\Delta m_{15}(B)>1.7$ (SN 2005bl, SN 2005ke, and SN 2006mr) which are marked with red circles in Figure 16. These SNe follow the behavior of the rest of the sample. This is substantiated by the results of Fits 3 and 4, done excluding these three SNe; the resulting fit parameters are in agreement within the uncertainties with those of Fits 1 and 2 , and the dispersions found are nearly the same. This is a remarkable result since the red colors of the fast-declining SNe Ia - which occur preferentially in E and S0 galaxies - are clearly largely intrinsic in origin, whereas the reddening of slower-declining $\mathrm{SNe}$ - which are most common in spiral galaxies - is presumably due at least in part to dust.

If it is assumed for the moment that the pseudocolors (after correction for Galactic reddening) vary due to reddening by dust in the host galaxy, the color term, $\beta$, can be converted to a totalto-selective absorption coefficient, $R_{V}$, adopting the $\mathrm{CCM}+\mathrm{O}$ reddening law and following the prescriptions of Appendix $\mathrm{B}$. The value of $R_{V}$ obtained from each fit is given in column 7 of Table 8. As is seen, all the fits thus far considered yield values of $R_{V} \approx 1.5$, which is significantly different from the standard Galactic value of 3.1. In the right-hand panels of Figure 16, the fits are labeled with the corresponding $R_{V}$ values. For comparison, the reddening vector predicted by $R_{V}=3.1$ is also plotted as a dotted line.

The Tripp (1998) model was also fit to the subset of best-observed SNe that meet the condition that $\left(B^{\max }-V^{\max }\right)<0.4$ mag. This excludes both the fast-declining objects, which are intrinsically red, and SNe 2005A and 2006X, which suffered heavy reddening due to dust. Fit 5 in Table 8 gives the results of such a fit. Once again, the zero point and slope of the luminosity vs. decline-decline rate relation $\left(M_{B}(0)\right.$ and $\left.b_{B}\right)$ do not change significantly in comparison with the previous fits. The luminosity-color slope, $\beta_{B}^{B V}$, does change slightly (by $\sim 1 \sigma$ ), resulting in an even lower value of $R_{V}$. These results are relevant to the study of high-redshift SNe Ia for which red (and therefore faint) objects [with $\left(B^{\max }-V^{\max }\right)>0.4 \mathrm{mag}$ ] are rarely observed (e.g., Kowalski et al. 2008).

The results of applying the Tripp (1998) model to $J$ versus $\Delta m_{15}(B)$ and $V-J$ for the sample of best-observed SNe with $J$-band coverage (21 objects) are given in Fit 6 of Table 8 , and are plotted in the lower part of Figure 16. Two additional fits are presented in Table 8 for the $J$ versus $\Delta m_{15}(B)$ and $V-J$ combination. In Fit 7, the two fast-declining SNe with coverage in $J$ are excluded. A slightly lower value of the slope $\beta_{V}^{V J}$ is obtained, with an almost negligible intrinsic dispersion of $\sigma_{\mathrm{SN}}=0.02 \mathrm{mag}$. Fit 8 employs a color cut of $\left(V^{\max }-J^{\max }\right)<0.0 \mathrm{mag}$, which is equivalent to limiting the sample to $\left(B^{\max }-V^{\max }\right)<0.4$ mag. Again, the best-fit parameters are in good agreement with those of Fit 7 , and the dispersions are likewise very similar.

Note that the interpretation of $\sigma_{\mathrm{SN}}$ as the intrinsic dispersion of the SN data about the model relies on the correct estimation of the measurement uncertainties and their covariances. An error in the treatment of the uncertainties would lead to different $\sigma_{\mathrm{SN}}$. Consequently, the fit parameters themselves could potentially change. In order to test this, the dependence of the fit parameters on the adopted uncertainties in the peak magnitudes, colors, and decline rates was examined. This was 
done by alternatively adding an arbitrary constant uncertainty on each quantity, up to 0.06 mag. While the values of $\sigma_{\mathrm{SN}}$ obtained were found to decrease with increasing uncertainties as expected, the best-fit values of the parameters remained well within the uncertainties.

In order to test the dependence of our results on the method used to derive light-curve parameters, we repeated the fits using peak magnitudes, decline rates and colors from template light-curve fits (see $\S 2.2$ ). We used the sample of best-observed SNe for which we performed both spline and template fits. The results are equivalent within the quoted errors to the ones presented above.

\subsection{Calibration Using Decline Rates and Extinction}

In this section, the color excesses derived in $\S 3$ are used to produce fits of absolute peak magnitude versus decline rate and reddening. It is explicitly assumed that the color excesses, $E(Y-Z)$, are due to dust and can be converted into an absorption in the $X$ band via $A_{X}=$ $R_{X}^{Y Z} E(Y-Z)$. This absorption is used to correct the observed peak magnitudes according to the model

$$
\tilde{\mu}_{X}=m_{X}-M_{X}(0)-b_{X}\left[\Delta m_{15}(B)-1.1\right]-R_{X}^{Y Z} E(Y-Z) .
$$

Note the similarity of this model to that of equation (6) of $\S 4.1$, especially if one considers that the color excesses derived in $\S 3.3$ are based on linear relationships between the observed pseudocolors and $\Delta m_{15}(B) 13$ Since the color excesses were obtained for SNe in the range $0.7<\Delta m_{15}(B)<1.7$ mag, the fast-declining $\mathrm{SNe}$ are necessarily excluded from the analysis of this section.

We first focus on fits done with $B$ and $E(B-V)$ data. To provide a reference, the sample was initially limited to the twelve SNe which were previously identified as having suffered little or no dust extinction (see $\S$ 3.2). These low-reddening objects were used to fit the model of equation (17) with the last term on the right-hand side set to zero - that is, without applying any reddening correction. The resulting parameters are given in Fit 1 of Table 9. These twelve SNe with low reddening yield a total dispersion of $0.19 \mathrm{mag}$, with an intrinsic dispersion $\sigma_{\mathrm{SN}}$ of the same amount.

Next, the entire SN sample was fit by first fixing $R_{V}$ to the standard Galactic value of 3.1. The results are given in Fit 2 of Table 9, where we solve only for $M_{B}(0)$ and $b_{B}$. This fit implies substantially higher peak luminosities and, most notably, a very large dispersion of $\sim 0.5$ mag. Thus, correcting for the observed reddening as if it were produced by typical Galactic interstellar dust yields a dispersion which is a factor of $\sim 2.5$ greater than that obtained for the subsample of low-reddening SNe.

Beginning with Fit 3 of Table $9, R_{B}^{B V}$ was allowed to be a free parameter. Here the full

\footnotetext{
${ }^{13}$ Indeed, as discussed in Appendix $\mathrm{A}$, the two treatments are mathematically equivalent.
} 
sample of SNe with $0.7<\Delta m_{15}(B)<1.7$ mag was employed, while in Fit 4 only the best-observed subsample was considered. The resulting parameters for these two fits are indistinguishable within the errors. The best-fit value of $R_{B}^{B V}$ in both cases is $\sim 2.8$, corresponding to $R_{V} \approx 1.5$. These two fits are shown in panels (a) and (b) of Figure 17.

The values of $R_{B}^{B V}$ derived in Fits 3 and 4 are strongly influenced by the two highly reddened SNe 2005A and 2006X. Fits 5 and 6 in Table 9 give solutions where these two SNe are excluded from the sample, with Fit 5 corresponding to the whole sample and Fit 6 to the best-observed SNe. As is seen, these fits still prefer a value of $R_{V} \approx 1.5$.

Bearing in mind the slight differences between photometric systems, the slope of the luminosity vs. decline-rate relation found for Fits 1-6 is in reasonable agreement with previously published results (Hamuy et al. 1996a; Altavilla et al. 2004; Prieto et al. 2006) 14. As shown in the left panels of Figure 17, the quadratic relationship found by Phillips et al. (1999) is also consistent with the data, although the CSP observations themselves are fit perfectly well by a simple linear relation.

Next, we considered fits using the peak magnitudes in ugriVYJHK$K_{s}$, combining each band with a suitable color excess. The choice of this color excess is somewhat arbitrary. For the ugr and $J H K_{s}$ bands, color excesses involving the filter itself were used. However, when this is done for the $i$ and $Y$ filters, the resulting fits yield significantly higher dispersions than are obtained when using $E(B-V)$. We attribute this to the poor quality of the template fits in the $i$ and $Y$ bands (see $\S$ 2.3), which not only introduces an error in the peak magnitude, but also in the color excess if the latter involves either of these bands.

Fits $7-15$ in Table 9 give the results for all bands other than $B$ using the sample of best-observed SNe. The best-fit values of $R_{V}$ range from 1.1 to 1.8 for ugriY J. For $H$ and $K_{s}$, somewhat larger values in the range $2.4-2.7$ are obtained, but with such large uncertainties that they are compatible with both low and standard values of $R_{V}$. A weighted average of the results for Fits $7-15$ yields $R_{V}=1.38 \pm 0.04$, which is consistent with the results obtained for $B$ vs. $E(B-V)$ (see Fit 4$)$. The scatter in the corrected magnitudes for Fits $7-15$ is in the range $0.12-0.16 \mathrm{mag}$, with the intrinsic components of the dispersion amounting to as small as 0.04 mag and as large as 0.13 mag.

\subsection{SNe Ia as Standard Candles in the Near-Infrared}

The set of NIR light curves presented here constitutes a significant contribution to the available data in the literature. The advantages of using NIR observations of SNe Ia to determine distances were demonstrated by Krisciunas, Phillips, \& Suntzeff (2004) and, more recently, by

\footnotetext{
${ }^{14}$ At maximum light, the $B-V$ colors of SNe Ia are close to zero, except for the most heavily reddened events. By definition, at $B-V=0.0 \mathrm{mag}$, magnitudes in the natural system are identical to those that have been color-corrected. Hence, comparing the CSP natural magnitudes at maximum light with those in systems where a color-term correction has been applied is a reasonable proposition.
} 
Wood-Vasey et al. (2008). Apart from being less affected by dust extinction and scattering which considerably reduces the effect of uncertainties in the color excesses and reddening law - the peak luminosities of SNe Ia in the NIR present a shallower dependence on decline rates than their optical counterparts.

Indeed, previous studies have shown no clear evidence for a correlation between luminosity and decline rate in the $J H K_{s}$ bands except for the very fastest declining SNe (Krisciunas et al. 2004b). The improved quality and coverage of the CSP NIR light curves allow a weak dependence of the luminosity on the decline rate to be discerned in the $J$ band. Fit 13 of Table 9 yields a slope of $b_{J}=0.58 \pm 0.09$ over $0.7<\Delta m_{15}(B)<1.7 \mathrm{mag}$, and is shown in panel (c) of Figure 17. In the $H$ band, the evidence is also suggestive for a weak correlation between luminosity and decline rate, although the significance of the measured slope is only $\sim 2 \sigma$ (see Fit 14 of Table 9). In a future paper (Kattner et al. 2009), we will use a larger sample of CSP SNe to test the reality of these relations.

To compare with previous studies, we compute the dispersion in absolute peak magnitudes without any correction for decline rate. Table 10 shows the results of weighted averages of the absolute peak magnitudes in $Y J H K_{s}$. These averages correspond to the sample of best-observed SNe, correcting for extinction using the values of $E(V-X)$ and $R_{X}^{Y Z}$ listed in Fits 12-15 of Table9. For comparison, averages were also taken limiting the sample to those SNe with low/moderate reddening (i.e., excluding SN 2005A and SN 2006X), and assuming two different values of $R_{X}^{Y Z}$ : zero, which is equivalent to ignoring host-galaxy extinction corrections, and the values of $R_{X}^{Y Z}$ that correspond to $R_{V}=3.1$.

The most striking implication of Table 10 is that, when the highly reddened SNe are excluded from the sample, the absolute magnitudes change only very slightly depending on the value of $R_{V}$. This illustrates the great advantage of working in the NIR, where lack of knowledge of the exact value of $R_{V}$ has little influence, and where dust-extinction corrections can essentially be ignored for all but the most heavily reddened SNe.

Table 10 shows that the dispersions in the absolute magnitudes are 0.18-0.21 mag in the $J$ and $H$ bands, depending only slightly on the assumed value of $R_{V}$. These are to be compared with the rms dispersions of $0.12 \mathrm{mag}$ and $0.16 \mathrm{mag}$, respectively, obtained when the absolute magnitudes are corrected for the decline-rate dependence (cf. Fits 13 and 14 of Table 9). For reference, Krisciunas et al. (2004b) obtained rms values (uncorrected for decline rate) of $0.13 \mathrm{mag}$ in $J$ and $0.15 \mathrm{mag}$ in $H$ for a sample of $22 \mathrm{SNe}$ Ia, and Wood-Vasey et al. (2008) found dispersions of $0.33 \mathrm{mag}$ in $J$ and $0.15 \mathrm{mag}$ in $H$ for $21 \mathrm{SNe}$.

In Table 11, the average absolute magnitudes at maximum of the best-observed subsample of CSP SNe are compared with those obtained by Krisciunas et al. (2004b). The values in $J$ and $H$ are consistent at the $\sim 2 \sigma$ level. In this same table, a comparison is also attempted with the absolute magnitudes at the epoch of $B$ maximum given by Wood-Vasey et al. (2008). For $J$ and $H$, these were derived using all the measurements available for the CSP SNe inside a bin of $[-2.5,2.5]$ 
rest-frame days with respect to $B$ maximum, converting these to absolute magnitudes as explained

below. A total of 33 and 28 data points entered into the calculations for $J$ and $H$, respectively. For the $K_{s}$ band, the relative scarcity of observations forces the use of a slightly larger bin size of $[-4,4]$ days in order to include 14 data points in the average. Again, the results in $J$ and $H$ are consistent with those of Wood-Vasey et al. (2008) at only the $\sim 1-2 \sigma$ level. In both cases, we suspect that the relatively poor agreement is due more to differences in the methods used to fit and combine the data rather than differences in the data themselves.

Figure 18 shows the absolute $i Y J H$ light curves for the sample of best-observed CSP SNe. The time axis is plotted as rest-frame days since maximum light in $B$. The Hubble-flow distances of equation (5) along with the Cepheid and SBF distances given in Table 7 are used to convert the observed magnitudes to absolute values. K-corrections (see $\S 2$ ), Galactic-extinction corrections with $R_{V}^{\text {Gal }}=3.1$, and host-galaxy extinction corrections using the measurements of color excesses $E(V-X)$ and the $R_{X}^{Y Z}$ parameters obtained from Fits 11-14 for $i Y J H$ have also been applied. For the fast-declining SNe 2005ke and $2006 \mathrm{mr}$, zero host-galaxy extinction is assumed.

Ignoring for the moment the two fast-declining SNe 2005ke and 2006mr, Figure 18 indicates that the smallest dispersion in absolute magnitude occurs around the time of the primary maximum in each filter. By the time of the minimum between the primary and secondary maximum, the dispersion increases in all bands. This observation is at odds with the suggestion by Kasen (2006) that the minimum of the $J$-band light curves of SNe Ia should show a low dispersion in luminosity. At epochs later than the second maximum, the data in $i Y J H$ show increasing spread due to differences in the decline rates among the SNe (see also $\S 2$ and Figure 2).

The two fast-declining events (SN 2005ke and SN 2006mr) do not reach maximum brightness until $\sim 5$ days after the epoch of $B$ maximum. Interestingly, Figure 18 shows that at this epoch, their $J$-band luminosities are similar to those of the rest of the SNe which are already declining from the first maximum.

\subsection{Combined Hubble Diagram}

The fits presented in $\S 4.2$ can be used to place the SNe with $0.7<\Delta m_{15}(B)<1.7$ mag in a Hubble diagram. Distance moduli were computed for each SN in ugriBVYJH using the parameters given in Table 9 for the fits to the best-observed SNe (Fits 4 and Fits 8-14). Note that Fit 7 involving $V$ and $E(B-V)$ was not included because it is mathematically equivalent to that involving $B$ and $E(B-V)$ (Fit 4$)$. The distance moduli for all available bands were then averaged for each SN. The full correlation matrix among distance moduli from all bands was estimated in order to weight the average. Correlation might be expected to arise from the fact that all fits involve the same decline-rate parameter $\Delta m_{15}(B)$, and in several cases, the same bands in the color-excess term. These averaged values are plotted versus the luminosity distance, $d_{L}$, in the top half of Figure 19. A combined scatter of $0.11 \mathrm{mag}$, or $\sim 5 \%$ in distance, is found for the 23 
best-observed SNe that are included in the diagram.

In the bottom panel where the residuals from the fit are plotted, it may be seen that the dispersion decreases with distance, implying that it is due predominantly to the peculiar velocities of the individual host galaxies. Plotted for reference as dotted lines is the spread predicted by the $1 \sigma$ dispersion of $382 \mathrm{~km} \mathrm{~s}^{-1}$, as derived by Wang et al. (2006) from an independent sample of 56 $\mathrm{SNe}$ Ia. In general, the distance modulus residuals are consistent with the latter value, although it is interesting that the four most-distant SNe all have negative residuals (meaning that they are overluminous). The most discrepant of these objects is SN 2004gu which, as discussed in C09, appears to have been spectroscopically similar to the peculiar SN Ia 2006gz, a slow-declining, overluminous SN Ia that may have resulted from the merger of two white dwarfs (Hicken et al. 2007). Both SNe displayed nearly identical decline rates $\left(\Delta m_{15}(B) \approx 0.7\right)$, and very similar luminosities $\left(M_{B} \approx-19.6 \mathrm{mag}\right)$. The other three SNe (2005ag, 2005ir, and 2006py) are all slow-decliners $\left(0.86 \leq \Delta m_{15}(B) \leq 1.02 \mathrm{mag}\right)$ and, therefore, luminous events. In principle, the luminosity vs. decline-rate relationship should correct for this. Nevertheless, SN 2005ag was discovered by the LOSS, and must have been at the limit of detection. Hence, Malmquist bias could explain its overluminous nature. However, SNe 2005ir and 2006py were discovered by the much deeper SDSS II survey which must have negligible bias at these redshifts. SN 2004gu had a moderately large color excess of $E(B-V)_{\max }=0.20$, but the other three events had color excesses very close to the median value of $E(B-V)_{\max }=0.05$ for the full subset of best-observed SNe. Hence, it is unlikely that the negative residuals in Figure 19 can be ascribed to inaccurate reddening corrections. Since the points for SNe 2005ag, 2005ir, and 2006py all lie within $1 \sigma$ of the expected velocity spread due to peculiar velocites, we consider small-number statistics to be the most likely explanation. Clearly it will be interesting to re-examine the Hubble diagram residuals once the full CSP sample has been produced.

Interestingly, although the Hubble diagram in Figure 19 was produced by averaging the distance moduli derived in each filter, the resulting dispersion is not much better than the dispersions obtained in the individual filters (cf. Table 9). The explanation for this is found in Figure 20, where the distance-modulus residuals in ugriY JHK are plotted versus the residual in $B$. A significant correlation is observed between the distance-modulus residuals in one band vs. those in another, particularly in the ugri bands. For this reason, the combined residuals do not reduce significantly the scatter in the diagram as compared with the fits to the individual bands.

We measured correlation coefficients, $r$, among residuals in Bugri to be 0.8-0.9, and 0.30.6 between any band and $Y J H K$. Most likely the lower correlation in the NIR is attributable to the difficulties of measuring precise peak magnitudes in these bands as discussed earlier. We compared the observed correlation coefficients with those estimated for each SN based on the measurement uncertainties and the form of the model in equation (7). We found the expected correlation coefficients to be $r<0.05$ for all cases, except between $B$ and $i$ bands where we estimate $r \approx 0.6$, and between $B$ and $Y$ where we expect $r \approx 0.3$. For these two cases, the high correlation may in part be due to the use of $E(B-V)$ in the color-excess term. For all the rest of 
the cases, the contribution to the correlations due to the fit model is negligible. We thus interpret the dispersion in Figure 19 to be mostly due to peculiar velocities. If this is correct, then the true precision of the $\mathrm{SN}$ distances is the dispersion about the relations in Figure 20, which for the ugri bands amounts to 0.06-0.09 mag in distance modulus, or 3-4\% in distance.

\section{DISCUSSION AND CONCLUSIONS}

This paper presents a first analysis of the light curves of 34 SNe Ia followed by the CSP and released by $\mathrm{C} 09$. The high photometric precision and dense time sampling of the observations, especially in the optical bands, has allowed an in-depth examination of the general properties of the light curves of SNe Ia in the ugriBVYJH bands. Subsamples of well-observed $\mathrm{SNe}-26$ in the optical and 9 in the NIR - were used to build a family of template light curves using a technique that allows one to interpolate among the template data, taking into account variations in the sampling, in a 3-D space parameterized by the epoch relative to maximum light, the magnitude relative to maximum, and the decline rate.

The availability of observations covering a wide range of wavelengths (from $u$ to $K_{s}$ ) offers the opportunity to make further progress on the difficult issue of correcting SN luminosities for extinction outside the Galaxy. However, depending on the approach taken, two quite different results are found. In the first case, a subsample of $15 \mathrm{SNe}$ assumed to have suffered little or no extinction in their host galaxies was used to measure color excesses for the whole sample. By plotting the ratios of color excesses involving optical and NIR filters and comparing the values predicted by the $\mathrm{CCM}+\mathrm{O}$ extinction law for interstellar dust, a value of the total-to-selective absorption coefficient of $R_{V} \approx 1.7$ was derived, which is significantly lower than the Galactic average of 3.1. However, this value is largely influenced by two very red objects in the sample, SNe 2005A and 2006X. When the same calculations are repeated after excluding these two objects, a value of $R_{V}=3.2 \pm 0.4$ is found, in agreement with the Galactic average.

An alternative way of estimating $R_{V}$ is to express the absolute magnitudes of the $\mathrm{SNe}$ as a two-parameter function of the decline rate and color, and then to use the Hubble-flow distances (or Cepheid and SBF distances) of the SNe to derive the best fit parameters through $\chi^{2}$ minimization. This method was first employed by Tripp (1998) and Tripp \& Branch (1999), who related the absolute magnitude in $B$ to the $B-V$ color and derived values of $R_{V} \approx 1-1.5$. When this same technique is applied to the CSP sample of SNe Ia using the peak magnitudes in $B$ and $J$ bands and the $B-V$ and $V-J$ colors, a value of $R_{V} \sim 1-2$ is obtained, regardless of whether the heavily reddened events, SNe 2005A and 2006X, are included or excluded. An equivalent analysis can also be carried out relating absolute magnitude to the decline rate and color excess. Performing such fits for all available bands (ugriBVYJHK$K_{s}$ ) again leads to systematically low values of the CCM+Olaw parameter $\left(R_{V} \approx 1.5\right)$ regardless of whether the two highly reddened SNe are included in the sample. 
These conflicting results on the value of $R_{V}$ reflect the variety of results found in the literature on this subject. Most early attempts to derive the average properties of the host-galaxy reddening indicated that $R_{V}$ was unusually low (see Branch \& Tammann 1992, and references therein). However, the available light curves at the time were largely photographic and there was considerable additional uncertainty regarding the nature of the intrinsic colors of SNe Ia at maximum light. The first modern study based on CCD data was made by Riess et al. (1996), who used the ratios of color excesses measured in $B-V, V-R$, and $V-I$ for a sample of $20 \mathrm{SNe}$ Ia to derive a value of $R_{V}=2.55 \pm 0.30$. This analysis differed from previous studies in that the intrinsic color variation as a function of decline rate was taken fully into account in deriving the color excesses. Similar values of $R_{V}$ have been found by Phillips et al. (1999), Altavilla et al. (2004), Reindl et al. (2005), and Wang et al. (2006). However, beginning with Tripp (1998), studies based on the technique of minimizing the dispersion in the Hubble diagram of SNe Ia have nearly uniformly led to values of $R_{V}$ in the range of $\sim 1-2$ (e.g., Tripp \& Branch 1999; Astier et al. 2006; Conley et al. 2007). If there is a pattern to these results, including our own in this paper, it would seem that attempts to measure $R_{V}$ via comparison of colors or color excesses tend to give larger values than the procedure of minimizing the scatter in the Hubble diagram with $R_{V}$ treated as a free parameter. Nevertheless, there are exceptions to this rule such as the recent paper by Nobili \& Goobar (2008), who studied the color evolution in $U-B, B-V, V-R$, and $R-I$ of 69 SNe Ia with moderate reddening and obtained a value of $R_{V}=1.01 \pm 0.25$.

Common sense suggests that at least some portion of the observed reddening of $\mathrm{SNe}$ Ia in spiral galaxies like the Milky Way must be due to dust in the interstellar medium of these galaxies. Although only a few studies have been carried out of the nature of the reddening law in external galaxies, these are generally consistent with $R_{V} \approx 3$, albeit with a large dispersion (Goudfrooij et al. 1994; Patil et al. 2007; Østman. Goobar, \& Mörtsell 2008). As shown in $\S$ 3.4.1, the ratios of the measured color excesses involving optical and NIR bands with respect to $E(B-V)$ are consistent with values of $R_{V} \approx 3$. As discussed by Krisciunas et al. (2007), inclusion of the NIR bands in this type of analysis improves considerably the accuracy with which $R_{V}$ can be measured. Hence, it is tempting to conclude that we are measuring reddening produced by dust with very similar properties to that found in the interstellar medium of the disk of the Milky Way. It will be interesting to see if this finding holds up as many more SNe Ia with reliable optical-NIR color excesses are added to the CSP sample.

If we are, indeed, observing host-galaxy reddening due to "normal" Galactic-type dust, then the fact that low values of $R_{V}(\sim 1-2)$ are obtained when it is treated as a free parameter in minimizing the dispersion in the Hubble diagram is puzzling. The implication is that there is an intrinsic dispersion in the colors of SNe Ia which is correlated with luminosity, but is independent of the decline rate. Our finding in $\S 3.4 .1$ that there is a small but systematic difference between color-excess measurements, $E(B-V)$, made at late epochs using the Lira law and those derived from the maximum light magnitudes, and that this difference correlates with color and perhaps also with absolute magnitude, may well be related to this. 
Very red SNe potentially allow the dust reddening law to be studied on an individual basis. Including the results for SN 2005A given in this paper, there are now six SNe Ia for which a determination of $R_{V}$ has been made from optical and NIR photometry. The results for these objects are summarized in Table 12. A weighted mean of the six determinations gives $R_{V}=1.6$, with a surprisingly small rms of 0.3 . The relatively narrow range of decline rates is also noteworthy, averaging $\Delta m_{15}(B)=1.2$ with a dispersion of only 0.1. Except for SN 2001el, the color excesses for these SNe are all very large. Jha, Riess, \& Kirshner (2007) found that the distribution of $E(B-V)$ for SNe Ia is well approximated by an exponential function with $\tau=0.138 \pm 0.023$ mag. Hence, values of $E(B-V)>1$ mag should be extremely rare, although it must be kept in mind that the sample of SNe analyzed by Jha et al. was culled from several sources with different selection criteria. Assuming that the Jha et al. distribution is correct, less than one SN for every thousand observed would be expected to have such a large amount of reddening - yet according to the Asiago Supernova Catalog 15 , the total number of nearby $(z<0.02)$ SNe Ia discovered from 1985 through 2008 was only 358. The fact that these heavily reddened SNe are much more common than expected, combined with the remarkable similarity of decline rates and $R_{V}$ values for the five objects in Table 12 with $E(B-V)>1$ mag, leads us to speculate that these events actually represent a physically distinct subclass of SNe Ia. Hence, while extremely interesting in themselves, these objects like SNe 2005A and 2006X are not representative of the class of SNe Ia employed in cosmological studies.

Wang (2005) has suggested that the presence of circumstellar dust distributed in a shell around the SN can produce reddening compatible with the observed low values of $R_{V}$. This hypothesis is supported by our observations of SNe 2005A and 2006X. Specifically, we find that the red colors of these two SNe are better matched by a model where multiple scattering of photons by circumstellar dust steepens the effective extinction law (Goobar 2008), than by the standard CCM+O Galactic reddening law with a low value of $R_{V}$.

Our results on the absolute peak magnitude calibrations for this new sample of CSP SNe confirm those obtained in previous studies, while extending the calibration to the optical ugri and the NIR $Y J H K_{s}$ bands. Fits to the subsample of best-observed SNe employing the Tripp (1998) model, which assumes the absolute magnitudes to be a two-parameter function of the decline rate and color, give rms dispersions of $0.12-0.15 \mathrm{mag}$. Remarkably, the same fits are found to apply equally well to either highly-reddened objects such as SNe 2005A and 2006X, or fast-declining, intrinsically-red events like 2005ke and $2006 \mathrm{mr}$. The simplicity of this method is appealing. We have also carried out fits which assume the absolute peak magnitudes to be a function of the decline rate and the measured color excesses. These give quite similar results to those obtained with the Tripp model. Fits to a large number of filters and colors give dispersions in the range of 0.12-0.20 mag for SNe with $0.7<\Delta m_{15}(B)<1.7$ mag.

The quality of our NIR light curves allows, for the first time, detection of a weak dependence

\footnotetext{
${ }^{15}$ http://web.oapd.inaf.it/supern/cat/ .
} 
of the $J$-band luminosity on decline rate. Combining the calibrations from all bands, we created a single Hubble diagram for the 23 best-observed SNe. Although the resulting scatter of $0.12 \mathrm{mag}$ (6\% in distance) is excellent, it does not represent a significant improvement over the precision obtained with individual filters. This is attributed to the fact that, for any particular SN, there is a significant correlation in the error in the distance moduli obtained using different filters. The source of these correlated errors appears to be the peculiar velocities of the SN host galaxies. If true, this implies that the derived distances to the SNe are actually precise to $3-4 \%$, making SNe Ia competitive with even Cepheid variables as extragalactic distance indicators.

Finally, the set of NIR light curves presented here confirms the advantages of working in this wavelength region for cosmological studies. Fit 8 of Table 8 and Fit 13 of Table 9 show that using either the $V-J$ color or color excess to correct the $J$ absolute magnitudes of the best-observed $\mathrm{SNe}$ (excluding the fast decliners) yields a dispersion of only 0.12 mag. Moreover, this result is insensitive to the exact form of the reddening law since the extinction corrections in the NIR are small. Note that the values derived for $\sigma_{r m S N}$ of $0.02-0.04$ mag suggest that the intrinsic dispersion in the $J$ band may be quite small. This is obviously a preliminary result which must be treated with caution - however, we will soon have a sample of $\sim 80 \mathrm{SNe}$ Ia to test this. If confirmed, it implies that if the observational uncertainties can be reduced, there is much to be gained by extending the rest-wavelength coverage of future dark-energy experiments to include the $J$ band.

This material is based upon work supported by the National Science Foundation (NSF) under grant AST-0306969. We also acknowledge support from Hubble Space Telescope grant GO-09860.07A from the Space Telescope Science Institute, which is operated by the Association of Universities for Research in Astronomy, Inc., under NASA contract NAS 5-26555. M.H. acknowledges support provided by NASA through Hubble Fellowship grant HST-HF-01139.01-A, by Fondecyt through grant 1060808, from Centro de Astrofísica FONDAP 15010003, and by the Center of Excellence in Astrophysics and Associated Technologies (PFB 06). G.F., M.H., and F.S. acknowledge support from the Millennium Center for Supernova Science through grant P06-045-F funded by "Programa Bicentenario de Ciencia y Tecnología de CONICYT" and "Programa Iniciativa Científica Milenio de MIDEPLAN." N.B.S. acknowledges the support of the Mitchell/Heep/Munnerlyn Chair in Astronomy at Texas A\&M University, and support though the Dean of the College of Sciences. A.V.F.'s supernova research has been funded by NSF grants AST-0607485 and AST-0908886, as well as by the TABASGO Foundation. We thank James Hughes for supporting our network of computers, and the technical staff of Las Campanas Observatory for its help during many observing nights. This research has made use of the NASA/IPAC Extragalactic Database (NED) which is operated by the Jet Propulsion Laboratory, California Institute of Technology, under contract with the National Aeronautics and Space Administration. 


\section{A. Chi-Squared Fitting}

The problem of calibrating the low-redshift CSP SNe in a particular band $X$ amounts to fitting a surface in a 3-dimensional parameter space defined by (1) a decline-rate parameter, $\Delta m_{15}(B)$; (2) a color parameter $c$, which could represent a pseudocolor at maximum, a color excess, or some other color indicator; and (3) the luminosity, $M_{X}$. For our analysis, we choose to fit this surface with a simple plane:

$$
M_{X}\left(\Delta m_{15}(B), c\right)=M_{X}(0)+b_{X}\left[\Delta m_{15}(B)-1.1\right]+\beta_{X} c .
$$

The $M_{X}$ values themselves are derived from the observed magnitudes $m_{X}$ by a distance modulus $\mu$ which, for the closest objects, can be derived from independent data (e.g., Cepheids, SBF). For the more distant objects, we use Hubble's law, assuming $H_{0}=72 \mathrm{~km} \mathrm{~s}^{-1} \mathrm{Mpc}^{-1}$. Our model for the observed magnitudes is therefore (cf. equations (6) and (7))

$$
\bar{m}_{X}=M_{X}(0)+b_{X}\left[\Delta m_{15}(B)-1.1\right]+\beta_{X} c+\mu .
$$

In the case where $c$ is interpreted as a color excess, we use the intrinsic color relation derived in $\oint 3.3$ to compute

$$
c \equiv E(Y-Z)=\left(m_{Y}-m_{Z}\right)-A_{0}-B_{0}\left[\Delta m_{15}(B)-1.1\right] .
$$

Substituting this in equation (A1) gives

$$
\bar{m}_{X}=\left(M_{X}(0)-\beta_{X} A_{0}\right)+\left(b_{X}-\beta_{X} B_{0}\right)\left[\Delta m_{15}(B)-1.1\right]+\beta_{X} c+\mu .
$$

In the case where $c$ is implemented as a pseudocolor ( $(4.1)$, the same model can be used, simply setting $A_{0}$ and $B_{0}$ to zero. In this way, we see that the two treatments of $c$ are mathematically equivalent. We also see very clearly that any uncertainties in the values of $A_{0}$ and $B_{0}$ are to be treated as systematic errors and should not be included in the statistical error budget for the color excesses. Rather, they should be included in the systematic error budget for the $M_{X}(0)$ and $b_{X}$.

Given a set of $N$ SNe with measured values $\left\{\mu_{i} ; m_{X i} ; \Delta m_{15}(B)_{i} ; c_{i}\right\}$ for $i=1, \ldots, N$, we construct the $\chi^{2}$ function

$$
\chi^{2}\left(M_{X}(0), b_{X}, \beta_{X}, \sigma_{S N}\right)=\sum_{i=1}^{N} \frac{\left(m_{X i}-\bar{m}_{X}\right)^{2}}{\sigma_{i}^{2}+\sigma_{S N}^{2}},
$$

where $\sigma_{i}^{2}$ are the measurement variances, and $\sigma_{S N}$ is an additional constant term to account for any possible intrinsic dispersion of the SN data which has not been taken into account by the model (see Tremaine et al. 2002). The distance moduli, $\mu_{i}$, are computed using equation (5). 


\section{A.1. Variances}

In computing the denominator of the $\chi^{2}$ function, care must be taken in estimating not only the variances of the data, but also the covariances between the different observables. We outline below how these variances and covariances are estimated. For each supernova, the total variance is (we omit the subscript $i$ for clarity)

$$
\begin{aligned}
\sigma^{2} & =\sigma^{2}(\mu)+\sigma^{2}\left(m_{X}\right)+b_{X}^{2} \sigma^{2}\left(\Delta m_{15}(B)\right)+\beta_{X}^{2} \sigma^{2}(c) \\
& -2 b_{X} \operatorname{cov}\left[m_{X}, \Delta m_{15}(B)\right]-2 \beta_{X} \operatorname{cov}\left[m_{X}, c\right] \\
& +2 b_{X} \beta_{X} \operatorname{cov}\left[\Delta m_{15}(B), c\right]
\end{aligned}
$$

where $\sigma^{2}(q)$ denotes the variance in quantity $q, \operatorname{cov}[p, q]$ denotes the covariance between quantity $p$ and $q$, and we have assumed $\operatorname{cov}[\mu, q]=0$. Furthermore, we assume the peak magnitudes in two different bands are uncorrelated $\left(\operatorname{cov}\left[m_{X}, m_{Y}\right]=\delta_{X Y} \sigma^{2}\left(m_{X}\right)\right)$. The three covariance terms in equation (A5) are computed as follows.

- For $\operatorname{cov}\left[m_{X}, \Delta m_{15}(B)\right]$, we recall that $\Delta m_{15}(B) \equiv m_{B}(t=15)-m_{B}(t=0)$ and so

$$
\begin{aligned}
\operatorname{cov}\left[m_{X}, \Delta m_{15}(B)\right] & =\operatorname{cov}\left[m_{X}, m_{B}(t=15)\right]-\operatorname{cov}\left[m_{X}, m_{B}(t=0)\right] \\
& =-\delta_{X B} \sigma^{2}\left(m_{X}\right)
\end{aligned}
$$

where we have assumed $\operatorname{cov}\left[m_{X}, m_{B}(t=15)\right]=0$. Note that we have neglected any possible correlated error introduced by the subtraction of the host-galaxy image in the $B$ band. As discussed by Contreras et al. (2009), such errors are less than the quoted statistical errors of the photometry.

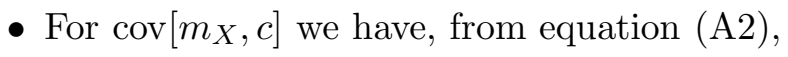

$$
\begin{aligned}
\operatorname{cov}\left[m_{X}, c\right] & =\operatorname{cov}\left[m_{X},\left(m_{Y}-m_{Z}\right)\right]-B_{0} \operatorname{cov}\left[m_{X}, \Delta m_{15}(B)\right] \\
& =\left(\delta_{X Y}-\delta_{X Z}+B_{0} \delta_{X B}\right) \sigma^{2}\left(m_{X}\right) .
\end{aligned}
$$

- For $\operatorname{cov}\left[\Delta m_{15}(B), c\right]$, we also have from equation (A2)

$$
\begin{aligned}
\sigma_{\left(\Delta m_{15}(B), c\right)} & =\operatorname{cov}\left[\Delta m_{15}(B),\left(m_{Y}-m_{Z}\right)\right]-\operatorname{cov}\left[\Delta m_{15}(B), B_{0} \Delta m_{15}(B)\right] \\
& =\left(\delta_{Z B}-\delta_{Y B}\right) \sigma^{2} m_{B}-B_{0} \sigma^{2}\left(\Delta m_{15}(B)\right) .
\end{aligned}
$$

Substituting equations (A6), (A7), and (A8) into equation (A5) for $\sigma^{2}$ we obtain the final expression for the measurement variances,

$$
\begin{aligned}
\sigma^{2} & =\sigma^{2}(\mu)+\left[1+2 \delta_{X B} b_{X}-2 \beta_{X}\left(\delta_{X Y}-\delta_{X Z}+B_{0} \delta_{X B}\right)\right] \sigma^{2}\left(m_{X}\right)+ \\
& +\left[b_{X}^{2}-2 b_{X} \beta_{X} B_{0}\right] \sigma^{2}\left(\Delta m_{15}(B)\right)+\beta_{X}^{2} \sigma_{c}^{2}+2 b_{X} \beta_{X}\left(\delta_{Z B}-\delta_{Y B}\right) \sigma^{2}\left(m_{B}\right),
\end{aligned}
$$

where we recall that $B_{0}=0$ for the case when $c$ represents a pseudocolor. 


\section{A.2. Solution}

The $\chi^{2}$ minimization is performed by analytically marginalizing equation (A4) over $M_{X}(0)$ (see Goliath et al. 2001) at each point in a grid of values of $\left\{b_{X} ; \beta_{X}\right\}$, followed by a search for the minimum value of $\chi^{2}$. This process is repeated for various values of $\sigma_{\mathrm{SN}}$-usually between 0 and $0.3 \mathrm{mag}$ - in order to find the value $\hat{\sigma}_{\mathrm{SN}}$ that produces a minimum reduced $\chi^{2}$ of $\chi_{\min }^{2} /(N-3)=1$.

Once $\hat{\sigma}_{\mathrm{SN}}$ is found, it is used for computing the probability density $\rho\left(\chi_{\text {marg }}^{2}\right)$ at each point in the grid of $\left\{b_{X} ; \beta_{X}\right\}$ values. This probability is given by

$$
\rho\left(b_{X}, \beta_{X}\right)=\exp \left\{\frac{-\chi_{\text {marg }}^{2}}{2}\right\} .
$$

Finally, the expectation value of each parameter, $\hat{\theta}$, in the grid of $\vec{\theta} \equiv\left\{M_{X}(0) ; b_{X} ; \beta_{X}\right\}$ is computed as the moment

$$
\hat{\theta}=\frac{\int \theta \rho(\vec{\theta}) d \vec{\theta}}{\int \rho(\vec{\theta}) d \vec{\theta}},
$$

and the variances in the fit parameters, $\sigma_{\hat{\theta}}$, are estimated as the second moment

$$
\sigma_{\hat{\theta}}^{2}=\frac{\int(\theta-\hat{\theta})^{2} \rho(\vec{\theta}) d \vec{\theta}}{\int \rho(\vec{\theta}) d \vec{\theta}}
$$

\section{B. Deriving $R_{V}$}

Here we explain how we compute the coefficients $a_{X}$ and $b_{X}$ for each band ugriBVYJHK that allow us to derive $R_{V}$ from the slopes $E(V-X)_{\max } / E(B-V)_{\max }$ of $\S$ 3.4.1, and from the fit parameters $R_{X}^{Y Z}$ of $\S 4.2$. (1989):

The reddening law is assumed to be a function of wavelength of the form given by Cardelli. Clavton. \& Mathis

$$
\frac{A_{\lambda}}{A_{V}}=a_{\lambda}+\frac{b_{\lambda}}{R_{V}}
$$

We simulate different amounts of extinction $A_{V}$ on the typical spectrum of a SN Ia at maximum light by multiplying equation (B1) into the spectrum. The spectrum we use is the template SN Ia spectrum of epoch 20 from Hsiao et al. (2007, 2009) which corresponds to maximum light in $B$. We perform the multiplication for various values of $R_{V}$, computing synthetic photometry from the resulting spectra using the transmission functions of the CSP filters described in C09. The $o b$ served absorptions, $A_{X}$ and $A_{V}$, are then given by the difference between the synthetic magnitudes obtained from the reddened template spectrum and those from the original unreddened one, for 
bands $X$ and $V$. We finally fit a linear relation between $A_{X}$ and $R_{V}$ as in equation (B1), and thus derive $a_{X}$ and $b_{X}$ for all bands ugriBVYJHK .

We have performed these calculations for the original Cardelli, Clavton, \& Mathis (1989) (CCM) reddening law, and also with the modifications introduced by O'Donnell (1994) (CCM+O). Columns 2-5 of Table 13 list the values of $a_{X}$ and $b_{X}$ obtained for each case. As will be explained below, these coefficients serve to convert an observed reddening measurement, such as those in $\S 3.4 .1$ and $\S 4.2$, to a value of $R_{V}$. We note that this would be a true value of $R_{V}$ - that is, the parameter defining the reddening law of equation (B1).

If we instead consider the observed values of $R_{V}$ derived from synthetic photometry as $R_{V}^{\text {obs }} \equiv$ $A_{V}^{\text {obs }} / E(B-V)^{\mathrm{obs}}$, we obtain different coefficients $a_{X}$ and $b_{X}$ from the fits of $A_{X}$ versus $R_{V}^{\text {obs }}$. These coefficients are listed in columns 6 and 7 of Table 13 for the case of the CCM+O law.

The formulae to convert observed slopes $E(V-X)_{\max } / E(B-V)_{\max }$ to $R_{V}$ are given in Appendix A of Krisciunas et al. (2006) [see their equations (A5) and (A6)]. We briefly explain here how to derive $R_{V}$ from fit parameters $R_{X}^{Y Z}$ of $\S 4.2$,

¿From the definition of $R_{X}=A_{X} / E(B-V), R_{X}^{Y Z}$ can be expressed as

$$
R_{X}^{Y Z}=\frac{R_{X}}{R_{Y}-R_{Z}}
$$

Substituting equation (B1) into this expression, and recalling that $A_{\lambda} / A_{V}$ is equivalent to $R_{\lambda} / R_{V}$, we derive

$$
R_{V}=-\frac{R_{X}^{Y Z}\left(b_{Y}-b_{Z}\right)-b_{X}}{R_{X}^{Y Z}\left(a_{Y}-a_{Z}\right)-a_{X}}
$$

By error propagation of the uncertainty in $R_{X}^{Y Z}$, the uncertainty in $R_{V}$ becomes

$$
\sigma_{R_{V}}=\left|\frac{\left(b_{Y}-b_{Z}\right)\left[R_{X}^{Y Z}\left(a_{Y}-a_{Z}\right)-a_{X}\right]+\left(a_{Y}-a_{Z}\right)\left[b_{X}-R_{X}^{Y Z}\left(b_{Y}-b_{Z}\right)\right]}{\left[R_{X}^{Y Z}\left(a_{Y}-a_{Z}\right)-a_{X}\right]^{2}}\right| \sigma_{R_{X}^{Y Z}} .
$$

\section{REFERENCES}

Allen, S. W., et al. 2004, MNRAS, 353, 457

Allen, S. W., et al. 2007, MNRAS, 383, 879

Altavilla, G., et al. 2004, MNRAS, 349, 1344

Arnett, W. D. 1982, ApJ, 253, 785 
Arnett, W. D., Branch, D., \& Wheeler, J. C. 1985, Nature, 314, 337

Astier, P., et al. 2006, A\&A, 447, 31

Blondin, S., et al. 2009, ApJ, 693, 207

Branch, D., \& Tammann, G. A. 1992, ARA\&A, 30, 359

Burns, C., et al. 2009, in preparation

Cantiello, M., et al. 2007, ApJ, 668, 130

Cardelli, J. A., Clayton, G. C., \& Mathis, J .S. 1989, ApJ, 345, 245

Cleveland, W. S. 1979, Journal of the American Statistical Association, 74, 829

Conley, A., et al. 2007, ApJ, 664, L13

Contreras, C., et al. 2009, (C09)

Dierckx, P. 1993, Curve and Surface Fitting with Splines, (Oxford: Oxford University Press)

Eisenstein, D.J. et al. 2005, ApJ, 633, 560

Elias-Rosa, N., et al. 2006, MNRAS, 369, 1880

Elias-Rosa, N., et al. 2008, MNRAS, 384, 107

Fitzpatrick, E. L., \& Massa, D. 2007, ApJ, 663, 320

Fixsen, D. J., et al. 1996, ApJ, 473, 576

Freedman, W. L., et al. 2001, ApJ, 553, 47

Freedman, W. L., et al. 2009, accepted to ApJ

Garnavich, P. M., et al. 2004, ApJ, 613, 1120

Goliath, M., et al. 2001, A\&A, 380, 6

Goobar, A. 2008, ApJ, 686, L103

Goudfrooij, P., de Jong, T., Hansen, L., \& Norgaard-Nielsen, H. U. 1994, MNRAS, 271, 833

Guy, J., Astier, P., Nobili, S., Regnault, N., \& Pain, R. 2005, A\&A, 443, 781

Hamuy, M., et al. 1996a, AJ, 112, 2391

Hamuy, M., et al. 1996b, AJ, 112, 2438

Hamuy, M., et al. 2006, PASP, 118, 2 (H06) 
Hicken, M., et al. 2007, ApJ, 669, L17

Höflich, P., Khokhlov, A. M., \& Wheeler, J. C. 1995, ApJ, 444, 831

Hsiao, E. Y., et al. 2007, ApJ, 663, 1187

Hsiao, E. Y., et al. 2009, in preparation

Jensen, J. B., et al. 2003, ApJ, 583, 712

Jha, S., et al. 2006, AJ, 131, 527

Jha, S., Riess, A. G., \& Kirshner, R. P. 2007, ApJ, 659, 122

Kasen, D. 2006, ApJ, 649, 939

Kattner, S., et al. 2009, in preparation

Kowalski, M., et al. 2008, ApJ, 686, 749

Krisciunas, K., Phillips, M. M., \& Suntzeff, N. B. 2004, ApJ, 602, 81

Krisciunas, K., et al. 2001, AJ, 122, 1616

Krisciunas, K., et al. 2004a, AJ, 127, 1664

Krisciunas, K., et al. 2004b, AJ, 128, 3034

Krisciunas, K., et al. 2006, AJ, 131, 1639

Krisciunas, K., et al. 2007, AJ, 133, 58

Landolt, A. U. 1992, AJ, 104, 340

Lira, P. 1995, Masters thesis, University of Chile

Nobili, S., \& Goobar, A. 2008, A\&A, 487, 19

Nugent, P., Phillips, M., Baron, E., Branch, D., \& Hauschildt, P. 1995, ApJ, 455, L147

O’Donnell, J. E. 1994, ApJ, 422, 158

Østman, L., Goobar, A., \& Mörtsell, E. 2008, A\&A, 485, 403

Patat, F., et al. 2007, Science, 317, 924

Patil, M. K., Pandey, S. K., Sahu, D. K., \& Kembhavi, A. 2007, A\&A, 461, 103

Perlmutter, S., et al. 1997, in Thermonuclear Supernova, ed. P. Ruiz-Lapuente, R. Canal, \& J. Isern (Dordrecht: Kluwer), 749 
Perlmutter, S., et al. 1999, ApJ, 517, 565

Persson, S. E., Madore, B. F., Krzeminski, W., Freedman, W. L., Roth, M., \& Murphy, D. C. 2004, AJ, 128, 2239

Phillips, M. M. 1993, ApJ, 413, L105

Phillips, M. M., et al. 1999, AJ, 118, 1766

Pinto, P. A., \& Eastman, R. G. 2000, ApJ, 530, 757

Prieto, J. L., Rest, A., \& Suntzeff, N. B. 2006, ApJ, 647, 501

Reindl, B., Tammann, G. A., Sandage, A., \& Saha, A. 2005, ApJ, 624, 532

Riess, A. G., et al. 1996, ApJ, 473, 588

Riess, A. G., et al. 1998, AJ, 116, 1009

Riess, A. G., et al. 2007, ApJ, 659, 98

Schlegel, D. J., Finkbeiner, D. P., \& Davis, M. 1998, ApJ, 500, 525

Smith, J. A., et al. 2002, AJ, 123, 2121

Spergel, D. N., et al. 2007, ApJS, 170, 377

Stritzinger, M., Mazzali, P. A., Sollerman, J., \& Benetti, S. 2006, A\&A, 460, 793

Tonry, J. L., et al. 2001, ApJ, 546, 681

Tremaine, S., et al. 2002, ApJ, 574, 740

Tripp, R. 1998, A\&A, 331,815

Tripp, R., \& Branch, D. 1999, ApJ, 525, 209

Wang, L. 2005, ApJ, 635, L33

Wang, L., et al. 2007, ApJ, 641, 50

Wang, X., et al. 2006, ApJ, 645, 488

Wang, X., et al. 2008a, ApJ, 675, 626

Wang, X., et al. 2008b, ApJ, 677, 1060

Wood-Vasey, W. M., et al. 2007, ApJ, 666, 694

Wood-Vasey, W. M., et al. 2008, ApJ, 689, 377 
Table 1.

\begin{tabular}{|c|c|c|c|c|c|c|}
\hline $\begin{array}{l}\text { SN } \\
(1)\end{array}$ & $\begin{array}{c}z_{\text {helio }} \\
(2)\end{array}$ & $\begin{array}{c}z_{\mathrm{CMB}} \\
(3)\end{array}$ & $\begin{array}{c}E(B-V) \\
\text { Gal (mag) } \\
(4)\end{array}$ & $\begin{array}{c}\Delta m_{15}(B) \\
\quad(\mathrm{mag}) \\
\quad(5)\end{array}$ & $\begin{array}{c}\langle\mu\rangle \\
(\mathrm{mag}) \\
(6)\end{array}$ & $\begin{array}{c}\text { Best } \\
\text { Obs.? } \\
(7)\end{array}$ \\
\hline $2004 \mathrm{ef}$ & 0.0310 & 0.0298 & 0.056 & $1.36(01)$ & $35.46(03)$ & yes \\
\hline $2004 \mathrm{eo}$ & 0.0157 & 0.0147 & 0.108 & $1.37(03)$ & $33.92(05)$ & yes \\
\hline $2004 \mathrm{ey}$ & 0.0158 & 0.0146 & 0.139 & $0.98(02)$ & $34.06(05)$ & yes \\
\hline $2004 \mathrm{gc}$ & 0.0321 & $0.0307^{\mathrm{a}}$ & 0.206 & $1.09(06)$ & $35.34(04)$ & no \\
\hline $2004 \mathrm{gs}$ & 0.0267 & 0.0275 & 0.031 & $1.54(01)$ & $35.51(03)$ & yes \\
\hline $2004 \mathrm{gu}$ & 0.0459 & 0.0469 & 0.026 & $0.80(04)$ & $36.41(10)$ & yes \\
\hline $2005 \mathrm{~A}$ & 0.0191 & 0.0184 & 0.030 & $1.17(03)$ & $34.46(06)$ & yes \\
\hline $2005 \mathrm{M}$ & 0.0220 & 0.0230 & 0.031 & $0.85(01)$ & $35.13(03)$ & yes \\
\hline $2005 \mathrm{~W}$ & 0.0089 & 0.0080 & 0.072 & $1.16(03)$ & $\ldots$ & yes \\
\hline $2005 \mathrm{ag}$ & 0.0794 & 0.0801 & 0.041 & $0.86(01)$ & $37.62(08)$ & yes \\
\hline 2005al & 0.0124 & 0.0133 & 0.055 & $1.22(04)$ & $34.01(06)$ & yes \\
\hline 2005am & 0.0079 & 0.0090 & 0.054 & $1.48(02)$ & & yes \\
\hline 2005be & 0.0350 & 0.0356 & 0.034 & $1.47(08)$ & $35.85(05)$ & no \\
\hline $2005 \mathrm{bg}$ & 0.0231 & 0.0242 & 0.032 & $1.02(08)$ & $35.08(06)$ & no \\
\hline 2005bl & 0.0241 & 0.0251 & 0.029 & $1.80(04)$ & $\ldots$ & yes \\
\hline 2005bo & 0.0139 & 0.0150 & 0.046 & $1.30(08)$ & $33.85(06)$ & yes \\
\hline $2005 \mathrm{el}$ & 0.0149 & 0.0149 & 0.114 & $1.29(01)$ & $33.92(05)$ & yes \\
\hline $2005 \mathrm{eq}$ & 0.0290 & 0.0284 & 0.074 & $0.72(02)$ & $35.44(03)$ & yes \\
\hline $2005 \mathrm{hc}$ & 0.0459 & 0.0450 & 0.029 & $0.90(01)$ & $36.51(05)$ & yes \\
\hline $2005 i q$ & 0.0340 & 0.0330 & 0.022 & $1.24(01)$ & $35.80(04)$ & yes \\
\hline 2005ir & 0.0764 & 0.0753 & 0.030 & $0.93(11)$ & $37.59(06)$ & yes \\
\hline $2005 \mathrm{kc}$ & 0.0151 & 0.0139 & 0.132 & $1.14(01)$ & $33.94(05)$ & yes \\
\hline 2005ke & 0.0049 & 0.0045 & 0.027 & $1.76(01)$ & $\ldots$ & yes \\
\hline 2005ki & 0.0192 & 0.0204 & 0.032 & $1.27(02)$ & $34.57(04)$ & yes \\
\hline 2005lu & 0.0320 & 0.0313 & 0.026 & $0.88(03)$ & $35.98(04)$ & no \\
\hline 2005na & 0.0263 & 0.0268 & 0.077 & $0.95(02)$ & $35.24(05)$ & yes \\
\hline $2006 \mathrm{D}$ & 0.0085 & 0.0097 & 0.046 & $1.42(01)$ & $\ldots$ & yes \\
\hline $2006 \mathrm{X}$ & 0.0052 & 0.0063 & 0.026 & $1.09(01)$ & $31.05(05)$ & yes \\
\hline $2006 a x$ & 0.0167 & 0.0180 & 0.050 & $0.99(02)$ & $34.30(04)$ & yes \\
\hline 2006bh & 0.0109 & 0.0105 & 0.026 & $1.41(01)$ & $33.25(07)$ & yes \\
\hline $2006 \mathrm{eq}$ & 0.0495 & 0.0484 & 0.048 & $1.66(06)$ & $36.54(02)$ & no \\
\hline $2006 \mathrm{gt}$ & 0.0448 & 0.0437 & 0.037 & $1.66(03)$ & $36.49(02)$ & yes \\
\hline $2006 \mathrm{mr}$ & 0.0059 & 0.0055 & 0.021 & $1.82(02)$ & $\ldots$ & yes \\
\hline 2006py & 0.0579 & 0.0567 & 0.065 & $1.02(03)$ & $36.88(02)$ & yes \\
\hline
\end{tabular}




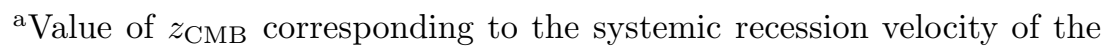
group ARP 327

Note. - Columns: (1) SN name; (2) Heliocentric redshift from the NASA/IPAC Extragalactic Database (NED); (3) Redshift in the frame of the 3K CMB (NED); (4) Galactic reddening (NED); (5) Observed $\Delta m_{15}(B)$; (6) Average distance modulus from fits of $\S 4.2$ (7) Does the SN belong to the "best-observed" sample?. 
Table 2. Light-curve parameters

\begin{tabular}{|c|c|c|c|c|c|c|c|}
\hline $\begin{array}{l}\text { Band } \\
\text { (1) }\end{array}$ & $\begin{array}{c}t^{\max } \\
-2,453,000 \\
(2)\end{array}$ & $\begin{array}{c}m^{\max } \\
(\mathrm{mag}) \\
(3)\end{array}$ & $\begin{array}{c}\Delta m_{t} \\
(\mathrm{mag}) \\
(4)\end{array}$ & \multicolumn{2}{|c|}{$\begin{array}{c}\text { Epoch } \\
\text { Range } \\
(5)\end{array}$} & $\begin{array}{c}\text { Npts. } \\
(6)\end{array}$ & $\begin{array}{c}\text { Fit } \\
\text { Method } \\
(7)\end{array}$ \\
\hline \multicolumn{8}{|c|}{ SN 2004ef } \\
\hline$B$ & $264.44 \pm 0.05$ & $17.059 \pm 0.005$ & $0.721 \pm 0.014$ & -8.5 & +63.2 & 44 & Spline \\
\hline$V$ & $266.07 \pm 0.03$ & $16.900 \pm 0.002$ & $1.120 \pm 0.008$ & -10.1 & +61.6 & 48 & Spline \\
\hline$u$ & $263.01 \pm 0.14$ & $17.791 \pm 0.012$ & $1.596 \pm 0.068$ & -6.2 & +56.8 & 41 & Spline \\
\hline$g$ & $264.82 \pm 0.06$ & $16.964 \pm 0.003$ & $1.077 \pm 0.009$ & -8.9 & +62.8 & 50 & Spline \\
\hline$r$ & $266.34 \pm 0.07$ & $16.862 \pm 0.003$ & $1.625 \pm 0.009$ & -10.3 & +61.3 & 47 & Spline \\
\hline$i$ & $261.21 \pm 0.08$ & $17.415 \pm 0.005$ & $1.199 \pm 0.013$ & -5.3 & +66.3 & 47 & Spline \\
\hline$Y$ & $261.25 \pm 0.03$ & $17.209 \pm 0.034$ & $\ldots$ & +8.8 & +69.5 & 7 & Templ. \\
\hline$J$ & $261.63 \pm 0.03$ & $17.230 \pm 0.080$ & $\ldots$ & +8.3 & +20.6 & 5 & Templ. \\
\hline$K$ & $262.47 \pm 0.03$ & $17.470 \pm 0.473$ & $\ldots$ & +7.5 & +19.8 & 6 & Templ. \\
\hline \multicolumn{8}{|c|}{ SN 2004eo } \\
\hline$B$ & $278.78 \pm 0.20$ & $15.500 \pm 0.008$ & $0.725 \pm 0.019$ & -12.0 & +61.8 & 39 & Spline \\
\hline$V$ & $280.45 \pm 0.11$ & $15.358 \pm 0.013$ & $1.070 \pm 0.021$ & -13.7 & +60.1 & 39 & Spline \\
\hline$u$ & $277.37 \pm 0.12$ & $16.064 \pm 0.015$ & $1.786 \pm 0.022$ & -10.7 & +43.5 & 37 & Spline \\
\hline$g$ & $279.23 \pm 0.17$ & $15.378 \pm 0.014$ & $1.107 \pm 0.021$ & -12.5 & +61.4 & 38 & Spline \\
\hline$r$ & $280.93 \pm 0.27$ & $15.358 \pm 0.009$ & $1.522 \pm 0.019$ & -14.2 & +59.7 & 39 & Spline \\
\hline$i$ & $275.88 \pm 0.17$ & $15.781 \pm 0.010$ & $1.029 \pm 0.020$ & -9.2 & +64.7 & 39 & Spline \\
\hline$Y$ & $274.78 \pm 0.07$ & $15.923 \pm 0.007$ & $\ldots$ & -5.1 & +31.3 & 15 & Spline \\
\hline$J$ & $274.67 \pm 0.02$ & $15.537 \pm 0.006$ & $\cdots$ & -5.0 & +62.9 & 17 & Spline \\
\hline$H$ & $274.71 \pm 0.17$ & $15.791 \pm 0.008$ & $\cdots$ & -5.0 & +62.8 & 18 & Spline \\
\hline$K$ & $275.66 \pm 0.20$ & $15.592 \pm 0.010$ & $\ldots$ & -5.9 & +30.4 & 14 & Spline \\
\hline \multicolumn{8}{|c|}{ SN 2004ey } \\
\hline$B$ & $304.39 \pm 0.10$ & $15.260 \pm 0.005$ & $0.466 \pm 0.007$ & -8.7 & +47.4 & 30 & Spline \\
\hline$V$ & $305.70 \pm 0.09$ & $15.215 \pm 0.006$ & $0.892 \pm 0.011$ & -10.0 & +46.1 & 32 & Spline \\
\hline$u$ & $303.24 \pm 0.08$ & $15.675 \pm 0.011$ & $1.277 \pm 0.015$ & -7.6 & +48.5 & 25 & Spline \\
\hline$g$ & $304.73 \pm 0.11$ & $15.214 \pm 0.003$ & $0.748 \pm 0.005$ & -9.1 & +47.1 & 26 & Spline \\
\hline$r$ & $305.95 \pm 0.13$ & $15.236 \pm 0.004$ & $1.149 \pm 0.007$ & -10.3 & +45.9 & 32 & Spline \\
\hline$i$ & $301.18 \pm 0.07$ & $15.761 \pm 0.003$ & $0.680 \pm 0.008$ & -5.6 & +50.6 & 32 & Spline \\
\hline$Y$ & $299.81 \pm 0.04$ & $15.785 \pm 0.018$ & $\ldots$ & +1.8 & +57.7 & 12 & Templ. \\
\hline$J$ & $301.06 \pm 0.04$ & $15.504 \pm 0.050$ & $\ldots$ & -0.5 & +56.4 & 15 & Templ. \\
\hline$H$ & $300.63 \pm 0.04$ & $15.743 \pm 0.022$ & $\ldots$ & +1.0 & +58.9 & 16 & Templ. \\
\hline$K$ & $302.05 \pm 0.04$ & $15.589 \pm 0.046$ & $\ldots$ & -0.4 & +55.4 & 14 & Templ. \\
\hline \multicolumn{8}{|c|}{ SN 2004gc } \\
\hline$B$ & $324.57 \pm 0.26$ & $17.467 \pm 0.028$ & $\ldots$ & +5.4 & +75.4 & 27 & Templ. \\
\hline$V$ & $325.85 \pm 0.26$ & $17.136 \pm 0.024$ & $\ldots$ & +4.1 & +74.1 & 28 & Templ. \\
\hline$g$ & $325.04 \pm 0.26$ & $17.328 \pm 0.026$ & $\cdots$ & +4.9 & +74.9 & 26 & Templ. \\
\hline$r$ & $325.78 \pm 0.26$ & $17.039 \pm 0.025$ & $\ldots$ & +4.2 & +74.1 & 28 & Templ. \\
\hline$i$ & $321.55 \pm 0.26$ & $17.446 \pm 0.026$ & $\ldots$ & +8.5 & +78.5 & 26 & Templ. \\
\hline \multicolumn{8}{|c|}{ SN 2004gs } \\
\hline$B$ & $356.18 \pm 0.04$ & $17.260 \pm 0.005$ & $0.854 \pm 0.022$ & -3.2 & +96.8 & 51 & Spline \\
\hline V & $358.64 \pm 0.18$ & $17.072 \pm 0.005$ & $1.368 \pm 0.025$ & -5.7 & +100.2 & 55 & Spline \\
\hline$u$ & $354.98 \pm 0.12$ & $17.983 \pm 0.018$ & $1.756 \pm 0.087$ & -2.1 & +35.7 & 23 & Spline \\
\hline$g$ & $356.79 \pm 0.04$ & $17.138 \pm 0.004$ & $1.294 \pm 0.034$ & -3.8 & +102.1 & 51 & Spline \\
\hline$r$ & $358.19 \pm 0.21$ & $17.046 \pm 0.005$ & $1.733 \pm 0.018$ & -5.2 & +100.7 & 52 & Spline \\
\hline
\end{tabular}


Table 2-Continued

\begin{tabular}{|c|c|c|c|c|c|c|c|}
\hline \multirow{2}{*}{$\begin{array}{l}\text { Band } \\
(1) \\
i\end{array}$} & \multirow{2}{*}{$\begin{array}{c}t^{\max } \\
-2,453,000 \\
(2) \\
355.76 \pm 0.05\end{array}$} & $\begin{array}{c}m^{\max } \\
(\mathrm{mag}) \\
(3)\end{array}$ & $\begin{array}{c}\Delta m_{t} \\
(\mathrm{mag}) \\
(4)\end{array}$ & \multicolumn{2}{|c|}{$\begin{array}{c}\text { Epoch } \\
\text { Range } \\
(5)\end{array}$} & \multirow{2}{*}{$\begin{array}{c}\text { Npts. } \\
(6)\end{array}$} & \multirow{2}{*}{$\begin{array}{c}\begin{array}{c}\text { Fit } \\
\text { Method } \\
(7)\end{array} \\
\text { Templ. }\end{array}$} \\
\hline & & $17.449 \pm 0.007$ & $1.469 \pm 0.214$ & -3.0 & +108.7 & & \\
\hline$J$ & $354.61 \pm 0.05$ & $17.323 \pm 0.017$ & & +8.4 & +60.6 & 19 & Templ. \\
\hline \multicolumn{8}{|c|}{ SN 2004gu } \\
\hline$B$ & $361.91 \pm 0.17$ & $17.554 \pm 0.022$ & $\ldots$ & -0.1 & +51.3 & 25 & Templ. \\
\hline$V$ & $363.26 \pm 0.78$ & $17.380 \pm 0.009$ & $\ldots$ & -5.2 & +45.5 & 26 & Spline \\
\hline$u$ & $360.54 \pm 0.17$ & $18.096 \pm 0.038$ & $\cdots$ & +1.4 & +35.9 & 14 & Templ. \\
\hline$g$ & $362.90 \pm 0.33$ & $17.417 \pm 0.008$ & $\ldots$ & -4.8 & +45.8 & 26 & Spline \\
\hline$r$ & $364.77 \pm 0.21$ & $17.340 \pm 0.005$ & $\cdots$ & -6.6 & +44.0 & 29 & Spline \\
\hline$i$ & $359.83 \pm 0.17$ & $17.916 \pm 0.013$ & $\cdots$ & -2.1 & +53.5 & 27 & Templ. \\
\hline \multicolumn{8}{|c|}{ SN 2005A } \\
\hline$B$ & $380.17 \pm 0.12$ & $18.262 \pm 0.008$ & $0.560 \pm 0.030$ & -3.5 & +29.8 & 23 & Spline \\
\hline$V$ & $382.72 \pm 0.71$ & $17.235 \pm 0.007$ & $0.956 \pm 0.018$ & -6.0 & +48.9 & 32 & Spline \\
\hline$u$ & $378.85 \pm 0.72$ & $19.933 \pm 0.043$ & $\ldots$ & -2.2 & +11.5 & 10 & Spline \\
\hline$g$ & $381.92 \pm 0.35$ & $17.830 \pm 0.007$ & $0.847 \pm 0.026$ & -5.2 & +49.6 & 36 & Spline \\
\hline$r$ & $381.50 \pm 0.17$ & $16.844 \pm 0.007$ & $1.283 \pm 0.015$ & -4.7 & +50.1 & 36 & Spline \\
\hline$i$ & $376.60 \pm 0.29$ & $17.184 \pm 0.014$ & $\ldots$ & +0.1 & +57.0 & 35 & Templ. \\
\hline$Y$ & $374.36 \pm 0.29$ & $16.398 \pm 0.026$ & $\cdots$ & +11.4 & +60.3 & 17 & Templ. \\
\hline$J$ & $375.62 \pm 0.29$ & $16.217 \pm 0.024$ & $\cdots$ & +10.1 & +56.0 & 17 & Templ. \\
\hline$H$ & $375.09 \pm 0.29$ & $16.404 \pm 0.034$ & $\ldots$ & +10.7 & +59.6 & 16 & Templ. \\
\hline \multicolumn{8}{|c|}{ SN 2005M } \\
\hline$B$ & $405.84 \pm 0.07$ & $16.046 \pm 0.004$ & $0.393 \pm 0.010$ & -7.9 & +73.1 & 56 & Spline \\
\hline$V$ & $407.88 \pm 0.16$ & $16.011 \pm 0.003$ & $0.838 \pm 0.014$ & -9.9 & +71.1 & 58 & Spline \\
\hline$u$ & $404.46 \pm 0.15$ & $16.359 \pm 0.018$ & $1.158 \pm 0.026$ & -7.5 & +52.0 & 47 & Spline \\
\hline$g$ & $406.28 \pm 0.08$ & $15.962 \pm 0.004$ & $0.662 \pm 0.015$ & -8.4 & +72.6 & 62 & Spline \\
\hline$r$ & $408.64 \pm 0.14$ & $16.102 \pm 0.006$ & $0.994 \pm 0.011$ & -10.7 & +70.3 & 59 & Spline \\
\hline$i$ & $402.98 \pm 0.11$ & $16.575 \pm 0.004$ & $0.688 \pm 0.013$ & -5.1 & +75.9 & 59 & Spline \\
\hline$Y$ & $402.11 \pm 0.14$ & $16.610 \pm 0.014$ & $0.071 \pm 0.021$ & -4.2 & +58.3 & 29 & Spline \\
\hline$J$ & $402.80 \pm 1.44$ & $16.495 \pm 0.048$ & $1.650 \pm 0.054$ & -4.9 & +57.6 & 32 & Spline \\
\hline$H$ & $401.92 \pm 1.93$ & $16.717 \pm 0.019$ & $0.216 \pm 0.024$ & -4.1 & +59.3 & 18 & Spline \\
\hline K & $403.22 \pm 0.02$ & $16.403 \pm 0.036$ & $\ldots$ & -5.6 & +60.7 & 15 & Templ. \\
\hline \multicolumn{8}{|c|}{ SN $2005 \mathrm{~W}$} \\
\hline$B$ & $412.08 \pm 0.11$ & $14.649 \pm 0.005$ & $0.573 \pm 0.024$ & -7.5 & +17.3 & 20 & Spline \\
\hline$V$ & $413.10 \pm 0.36$ & $14.235 \pm 0.009$ & $\ldots$ & -8.5 & +16.3 & 21 & Spline \\
\hline$u$ & $410.86 \pm 0.16$ & $15.014 \pm 0.007$ & $1.277 \pm 0.119$ & -6.3 & +16.5 & 18 & Spline \\
\hline$g$ & $412.55 \pm 0.14$ & $14.330 \pm 0.009$ & $0.791 \pm 0.027$ & -7.9 & +16.8 & 20 & Spline \\
\hline$r$ & $413.88 \pm 0.53$ & $14.196 \pm 0.009$ & $\ldots$ & -9.3 & +15.5 & 21 & Spline \\
\hline$i$ & $408.44 \pm 0.43$ & $14.637 \pm 0.009$ & $\ldots$ & -3.9 & +20.9 & 19 & Spline \\
\hline \multicolumn{8}{|c|}{ SN 2005ag } \\
\hline$B$ & $413.53 \pm 0.13$ & $18.593 \pm 0.006$ & $\ldots$ & -0.8 & +73.2 & 44 & Templ. \\
\hline$V$ & $415.14 \pm 0.13$ & $18.550 \pm 0.006$ & $\cdots$ & -2.5 & +71.4 & 44 & Templ. \\
\hline$g$ & $413.98 \pm 0.13$ & $18.551 \pm 0.005$ & $\ldots$ & -1.3 & +72.6 & 44 & Templ. \\
\hline$r$ & $415.70 \pm 0.13$ & $18.631 \pm 0.005$ & $\cdots$ & -3.1 & +70.8 & 46 & Templ. \\
\hline$i$ & $411.23 \pm 0.13$ & $18.955 \pm 0.020$ & $\ldots$ & +1.7 & +75.6 & 44 & Templ. \\
\hline$Y$ & $408.79 \pm 0.13$ & $19.325 \pm 0.021$ & $\cdots$ & +10.9 & +45.7 & 10 & Templ. \\
\hline
\end{tabular}


Table 2-Continued

\begin{tabular}{|c|c|c|c|c|c|c|c|}
\hline \multirow{2}{*}{$\begin{array}{l}\text { Band } \\
(1) \\
J\end{array}$} & \multirow{2}{*}{$\begin{array}{c}t^{\max } \\
-2,453,000 \\
(2)\end{array}$} & $\begin{array}{c}m^{\max } \\
(\operatorname{mag}) \\
(3)\end{array}$ & $\begin{array}{c}\Delta m_{t} \\
(\mathrm{mag}) \\
(4)\end{array}$ & \multicolumn{2}{|c|}{$\begin{array}{c}\text { Epoch } \\
\text { Range } \\
(5)\end{array}$} & \multirow{2}{*}{$\begin{array}{c}\text { Npts. } \\
(6) \\
10\end{array}$} & \multirow{2}{*}{$\begin{array}{c}\begin{array}{c}\text { Fit } \\
\text { Method } \\
(7)\end{array} \\
\text { Templ. }\end{array}$} \\
\hline & & $19.255 \pm 0.037$ & $\ldots$ & +9.4 & +44.2 & & \\
\hline$H$ & $409.65 \pm 0.13$ & $19.067 \pm 0.055$ & $\ldots$ & +10.0 & +44.8 & 10 & Templ. \\
\hline \multicolumn{8}{|c|}{ SN 2005al } \\
\hline$B$ & $430.84 \pm 0.30$ & $15.084 \pm 0.006$ & $0.630 \pm 0.009$ & -1.1 & +80.9 & 35 & Spline \\
\hline$V$ & $431.57 \pm 0.38$ & $15.076 \pm 0.005$ & $1.066 \pm 0.010$ & -1.8 & +80.2 & 36 & Spline \\
\hline$u$ & $429.12 \pm 0.11$ & $15.530 \pm 0.014$ & $1.565 \pm 0.017$ & +0.6 & +84.7 & 35 & Templ. \\
\hline$g$ & $430.65 \pm 0.15$ & $15.033 \pm 0.004$ & $0.935 \pm 0.009$ & -0.9 & +81.1 & 35 & Spline \\
\hline$r$ & $431.91 \pm 0.48$ & $15.122 \pm 0.007$ & $1.573 \pm 0.012$ & -2.1 & +79.9 & 36 & Spline \\
\hline$i$ & $427.98 \pm 0.11$ & $15.729 \pm 0.009$ & $\ldots$ & +1.8 & +85.9 & 35 & Templ. \\
\hline$Y$ & $426.43 \pm 0.11$ & $15.677 \pm 0.013$ & $\ldots$ & +7.5 & +36.8 & 12 & Templ. \\
\hline$J$ & $427.05 \pm 0.11$ & $15.531 \pm 0.015$ & $\ldots$ & +6.9 & +36.2 & 13 & Templ. \\
\hline$H$ & $426.67 \pm 0.11$ & $15.808 \pm 0.017$ & $\ldots$ & +7.3 & +36.6 & 13 & Templ. \\
\hline$K$ & $428.05 \pm 0.11$ & $15.720 \pm 0.064$ & $\ldots$ & +5.9 & +35.2 & 9 & Templ. \\
\hline \multicolumn{8}{|c|}{ SN 2005am } \\
\hline$B$ & $436.86 \pm 0.07$ & $13.857 \pm 0.003$ & $0.775 \pm 0.007$ & -4.3 & +75.0 & 37 & Spline \\
\hline$V$ & $438.45 \pm 0.12$ & $13.760 \pm 0.003$ & $1.256 \pm 0.007$ & -5.8 & +73.4 & 37 & Spline \\
\hline$u$ & $435.63 \pm 0.05$ & $14.408 \pm 0.005$ & $1.750 \pm 0.014$ & -3.0 & +76.2 & 37 & Spline \\
\hline$g$ & $437.28 \pm 0.03$ & $13.780 \pm 0.002$ & $1.152 \pm 0.008$ & -4.7 & +74.6 & 38 & Spline \\
\hline$r$ & $438.43 \pm 0.10$ & $13.742 \pm 0.005$ & $1.741 \pm 0.009$ & -5.8 & +73.4 & 38 & Spline \\
\hline$i$ & $435.36 \pm 0.08$ & $14.254 \pm 0.010$ & $\ldots$ & -2.8 & +77.7 & 36 & Templ. \\
\hline$J$ & $434.10 \pm 0.08$ & $14.009 \pm 0.014$ & $\ldots$ & -0.4 & +28.7 & 12 & Templ. \\
\hline$H$ & $434.26 \pm 0.08$ & $14.132 \pm 0.011$ & $\ldots$ & -0.6 & +28.5 & 14 & Templ. \\
\hline$K$ & $434.50 \pm 0.08$ & $14.135 \pm 0.043$ & $\ldots$ & -0.8 & +28.2 & 14 & Templ. \\
\hline \multicolumn{8}{|c|}{ SN 2005be } \\
\hline$B$ & $460.59 \pm 0.28$ & $17.023 \pm 0.035$ & $\ldots$ & +7.6 & +32.3 & 13 & Templ. \\
\hline$V$ & $462.12 \pm 0.28$ & $16.974 \pm 0.030$ & $\ldots$ & +6.0 & +30.7 & 13 & Templ. \\
\hline$u$ & $459.00 \pm 0.28$ & $17.364 \pm 0.042$ & $\ldots$ & +9.2 & +25.7 & 9 & Templ. \\
\hline$g$ & $461.12 \pm 0.28$ & $16.950 \pm 0.032$ & $\ldots$ & +7.0 & +31.7 & 12 & Templ. \\
\hline$r$ & $461.90 \pm 0.28$ & $17.054 \pm 0.028$ & $\cdots$ & +6.2 & +30.9 & 12 & Templ. \\
\hline$i$ & $458.83 \pm 0.28$ & $17.630 \pm 0.020$ & $\ldots$ & +9.4 & +34.1 & 12 & Templ. \\
\hline \multicolumn{8}{|c|}{ SN 2005bg } \\
\hline$B$ & $470.70 \pm 0.33$ & $16.010 \pm 0.042$ & $\ldots$ & +1.0 & +42.9 & 16 & Templ. \\
\hline$V$ & $471.89 \pm 0.33$ & $15.941 \pm 0.044$ & $\cdots$ & -0.3 & +41.7 & 16 & Templ. \\
\hline$u$ & $469.24 \pm 0.33$ & $16.400 \pm 0.037$ & $\ldots$ & +3.5 & +22.9 & 13 & Templ. \\
\hline$g$ & $471.13 \pm 0.33$ & $15.997 \pm 0.043$ & $\ldots$ & +1.6 & +42.5 & 15 & Templ. \\
\hline$r$ & $472.08 \pm 0.33$ & $16.082 \pm 0.046$ & $\ldots$ & +0.6 & +41.5 & 15 & Templ. \\
\hline$i$ & $467.74 \pm 0.33$ & $16.676 \pm 0.052$ & $\ldots$ & +5.1 & +45.9 & 15 & Templ. \\
\hline \multicolumn{8}{|c|}{ SN 2005bl } \\
\hline$B$ & $484.25 \pm 0.36$ & $18.336 \pm 0.031$ & $\ldots$ & -7.5 & +27.6 & 12 & Spline \\
\hline$V$ & $485.39 \pm 0.11$ & $17.753 \pm 0.017$ & $\cdots$ & -8.6 & +26.5 & 12 & Spline \\
\hline$u$ & $481.42 \pm 0.67$ & $19.784 \pm 0.076$ & $\ldots$ & -4.7 & +9.9 & 10 & Spline \\
\hline$g$ & $484.03 \pm 0.53$ & $18.129 \pm 0.057$ & $\cdots$ & -7.3 & +27.9 & 12 & Spline \\
\hline$r$ & $486.31 \pm 0.12$ & $17.694 \pm 0.004$ & $\ldots$ & -9.5 & +25.7 & 12 & Spline \\
\hline$i$ & $486.50 \pm 0.09$ & $17.791 \pm 0.006$ & $\cdots$ & -9.6 & +25.5 & 12 & Spline \\
\hline
\end{tabular}


Table 2-Continued

\begin{tabular}{|c|c|c|c|c|c|c|c|}
\hline $\begin{array}{l}\text { Band } \\
\text { (1) }\end{array}$ & $\begin{array}{c}t^{\max } \\
-2,453,000 \\
(2)\end{array}$ & $\begin{array}{c}m^{\max } \\
(\mathrm{mag}) \\
(3)\end{array}$ & $\begin{array}{c}\Delta m_{t} \\
(\mathrm{mag}) \\
(4)\end{array}$ & \multicolumn{2}{|c|}{$\begin{array}{c}\text { Epoch } \\
\text { Range } \\
(5)\end{array}$} & $\begin{array}{c}\text { Npts. } \\
(6)\end{array}$ & $\begin{array}{c}\text { Fit } \\
\text { Method } \\
(7)\end{array}$ \\
\hline \multicolumn{8}{|c|}{ SN 2005bo } \\
\hline$B$ & $479.76 \pm 0.08$ & $15.845 \pm 0.009$ & $\ldots$ & -1.0 & +32.6 & 9 & Spline \\
\hline V & $480.80 \pm 0.14$ & $15.515 \pm 0.015$ & $\ldots$ & -2.0 & +31.5 & 10 & Spline \\
\hline$u$ & $477.62 \pm 0.14$ & $16.463 \pm 0.036$ & $\ldots$ & +1.1 & +14.3 & 8 & Templ. \\
\hline$g$ & $480.29 \pm 0.22$ & $15.701 \pm 0.007$ & $\ldots$ & -1.5 & +32.0 & 9 & Spline \\
\hline$r$ & $480.95 \pm 0.28$ & $15.457 \pm 0.010$ & $\cdots$ & -2.2 & +31.4 & 9 & Spline \\
\hline$i$ & $476.50 \pm 0.14$ & $15.790 \pm 0.027$ & $\ldots$ & +2.3 & +36.8 & 9 & Templ. \\
\hline \multicolumn{8}{|c|}{ SN 2005el } \\
\hline$B$ & $647.24 \pm 0.05$ & $15.290 \pm 0.008$ & $0.657 \pm 0.010$ & -7.2 & +82.3 & 25 & Spline \\
\hline$V$ & $647.89 \pm 0.08$ & $15.235 \pm 0.005$ & $1.121 \pm 0.007$ & -7.9 & +81.6 & 24 & Spline \\
\hline$u$ & $645.52 \pm 0.03$ & $15.664 \pm 0.006$ & $1.540 \pm 0.017$ & -5.6 & +84.0 & 24 & Spline \\
\hline$g$ & $647.34 \pm 0.04$ & $15.167 \pm 0.010$ & $1.039 \pm 0.011$ & -7.4 & +82.2 & 24 & Spline \\
\hline$r$ & $646.89 \pm 0.08$ & $15.290 \pm 0.009$ & $1.485 \pm 0.014$ & -6.9 & +82.6 & 24 & Spline \\
\hline$i$ & $643.75 \pm 0.17$ & $15.714 \pm 0.006$ & $1.285 \pm 0.013$ & -3.8 & +85.7 & 25 & Spline \\
\hline$Y$ & $642.35 \pm 0.03$ & $15.514 \pm 0.010$ & $0.377 \pm 0.018$ & -1.5 & +72.3 & 42 & Spline \\
\hline$J$ & $642.81 \pm 0.13$ & $15.584 \pm 0.007$ & $2.443 \pm 0.044$ & -1.9 & +71.8 & 36 & Spline \\
\hline$H$ & $642.22 \pm 0.13$ & $15.642 \pm 0.009$ & $0.995 \pm 0.032$ & -1.3 & +72.4 & 27 & Spline \\
\hline$K$ & $644.59 \pm 0.07$ & $15.572 \pm 0.135$ & $\ldots$ & -2.9 & +37.6 & 4 & Templ. \\
\hline \multicolumn{8}{|c|}{ SN 2005eq } \\
\hline$B$ & $654.17 \pm 0.14$ & $16.591 \pm 0.006$ & $0.321 \pm 0.011$ & -3.3 & +94.7 & 27 & Spline \\
\hline$V$ & $655.68 \pm 0.25$ & $16.453 \pm 0.007$ & $0.793 \pm 0.017$ & -4.8 & +93.3 & 27 & Spline \\
\hline$u$ & $652.10 \pm 0.09$ & $16.914 \pm 0.013$ & $0.894 \pm 0.344$ & -1.3 & +62.4 & 19 & Templ. \\
\hline$g$ & $654.46 \pm 0.16$ & $16.496 \pm 0.005$ & $0.562 \pm 0.008$ & -3.6 & +94.5 & 27 & Spline \\
\hline$r$ & $656.16 \pm 0.13$ & $16.485 \pm 0.006$ & $0.966 \pm 0.013$ & -5.2 & +92.8 & 27 & Spline \\
\hline$i$ & $652.23 \pm 0.09$ & $17.032 \pm 0.050$ & $\ldots$ & -1.4 & +102.4 & 27 & Templ. \\
\hline$Y$ & $649.80 \pm 0.09$ & $16.902 \pm 0.050$ & $\cdots$ & -2.0 & +62.7 & 28 & Templ. \\
\hline$J$ & $650.64 \pm 0.09$ & $16.872 \pm 0.029$ & $\cdots$ & -2.9 & +61.8 & 26 & Templ. \\
\hline$H$ & $650.24 \pm 0.09$ & $17.090 \pm 0.039$ & $\cdots$ & -1.5 & +43.8 & 11 & Templ. \\
\hline$K$ & $651.28 \pm 0.09$ & $16.611 \pm 0.073$ & $\ldots$ & +2.6 & +2.6 & 2 & Templ. \\
\hline \multicolumn{8}{|c|}{ SN 2005hc } \\
\hline$B$ & $667.14 \pm 0.08$ & $17.426 \pm 0.004$ & $0.425 \pm 0.018$ & -4.2 & +80.8 & 24 & Spline \\
\hline$V$ & $668.88 \pm 0.65$ & $17.403 \pm 0.006$ & $0.822 \pm 0.020$ & -5.9 & +82.9 & 25 & Spline \\
\hline$u$ & $666.28 \pm 0.16$ & $17.875 \pm 0.009$ & $1.066 \pm 0.037$ & -3.4 & +23.4 & 11 & Spline \\
\hline$g$ & $667.69 \pm 0.16$ & $17.368 \pm 0.005$ & $0.700 \pm 0.024$ & -4.7 & +84.0 & 23 & Spline \\
\hline$r$ & $669.01 \pm 0.13$ & $17.469 \pm 0.005$ & $1.028 \pm 0.012$ & -6.0 & +82.8 & 23 & Spline \\
\hline$i$ & $665.13 \pm 0.08$ & $18.143 \pm 0.008$ & $\ldots$ & -2.5 & +79.1 & 22 & Templ. \\
\hline$Y$ & $662.73 \pm 0.08$ & $17.786 \pm 0.050$ & $\cdots$ & -1.1 & +50.2 & 23 & Templ. \\
\hline$J$ & $664.16 \pm 0.08$ & $17.936 \pm 0.046$ & $\cdots$ & -2.6 & +48.7 & 22 & Templ. \\
\hline$H$ & $662.92 \pm 0.08$ & $17.999 \pm 0.103$ & $\ldots$ & -1.2 & +35.5 & 13 & Templ. \\
\hline \multicolumn{8}{|c|}{ SN 2005iq } \\
\hline$B$ & $687.78 \pm 0.06$ & $16.861 \pm 0.007$ & $0.633 \pm 0.012$ & -5.1 & +65.5 & 20 & Spline \\
\hline$V$ & $689.70 \pm 0.17$ & $16.872 \pm 0.010$ & $1.139 \pm 0.020$ & -6.9 & +63.7 & 21 & Spline \\
\hline$u$ & $686.49 \pm 0.10$ & $17.227 \pm 0.010$ & $1.329 \pm 0.041$ & -3.8 & +58.1 & 17 & Spline \\
\hline$g$ & $688.16 \pm 0.07$ & $16.804 \pm 0.007$ & $0.988 \pm 0.011$ & -5.4 & +65.2 & 20 & Spline \\
\hline
\end{tabular}


Table 2-Continued

\begin{tabular}{|c|c|c|c|c|c|c|c|}
\hline \multirow{2}{*}{$\begin{array}{l}\text { Band } \\
(1) \\
r\end{array}$} & \multirow{2}{*}{$\begin{array}{c}t^{\max } \\
-2,453,000 \\
(2)\end{array}$} & \multirow{2}{*}{$\begin{array}{c}\begin{array}{c}m^{\max } \\
(\mathrm{mag}) \\
(3)\end{array} \\
16.957 \pm 0.007\end{array}$} & \multirow{2}{*}{$\begin{array}{c}\Delta m_{t} \\
(\mathrm{mag}) \\
(4)\end{array}$} & \multicolumn{2}{|c|}{$\begin{array}{c}\text { Epoch } \\
\text { Range } \\
(5)\end{array}$} & \multirow{2}{*}{$\begin{array}{c}\text { Npts. } \\
(6)\end{array}$} & \multirow{2}{*}{$\begin{array}{c}\begin{array}{c}\text { Fit } \\
\text { Method } \\
(7)\end{array} \\
\text { Spline }\end{array}$} \\
\hline & & & & -6.3 & +64.3 & & \\
\hline$i$ & $684.38 \pm 0.09$ & $17.421 \pm 0.011$ & $1.111 \pm 0.025$ & -1.8 & +68.8 & 20 & Spline \\
\hline$Y$ & $683.67 \pm 0.06$ & $17.224 \pm 0.050$ & $\ldots$ & +2.0 & +50.7 & 22 & Templ. \\
\hline$J$ & $684.33 \pm 0.06$ & $17.402 \pm 0.045$ & $\ldots$ & +1.3 & +50.0 & 19 & Templ. \\
\hline \multirow[t]{2}{*}{$H$} & $684.21 \pm 0.06$ & $17.363 \pm 0.050$ & $\ldots$ & +1.7 & +39.0 & 20 & Templ. \\
\hline & & & SN 2005ir & & & & \\
\hline$B$ & $685.60 \pm 1.26$ & $18.518 \pm 0.024$ & $0.483 \pm 0.030$ & -2.8 & +40.0 & 12 & Spline \\
\hline V & $688.25 \pm 1.09$ & $18.429 \pm 0.026$ & $1.015 \pm 0.034$ & -5.2 & +48.6 & 14 & Spline \\
\hline$u$ & $683.37 \pm 0.23$ & $18.889 \pm 0.054$ & $\ldots$ & +0.3 & +22.0 & 8 & Templ. \\
\hline$g$ & $685.81 \pm 0.61$ & $18.497 \pm 0.014$ & $0.776 \pm 0.024$ & -3.0 & +20.3 & 10 & Spline \\
\hline$r$ & $687.61 \pm 0.33$ & $18.512 \pm 0.021$ & $\ldots$ & -4.6 & +18.7 & 10 & Spline \\
\hline \multirow[t]{2}{*}{$i$} & $681.96 \pm 0.23$ & $19.163 \pm 0.053$ & $0.739 \pm 0.126$ & +0.7 & +61.3 & 12 & Templ. \\
\hline & & & SN 2005kc & & & & \\
\hline$B$ & $697.75 \pm 0.06$ & $16.033 \pm 0.005$ & $0.560 \pm 0.012$ & -10.1 & +30.3 & 13 & Spline \\
\hline V & $699.56 \pm 0.12$ & $15.742 \pm 0.006$ & $1.028 \pm 0.012$ & -11.9 & +28.5 & 13 & Spline \\
\hline$u$ & $696.50 \pm 0.16$ & $16.704 \pm 0.010$ & $1.465 \pm 0.034$ & -8.8 & +23.7 & 11 & Spline \\
\hline$g$ & $698.39 \pm 0.08$ & $15.886 \pm 0.005$ & $0.894 \pm 0.011$ & -10.7 & +29.7 & 13 & Spline \\
\hline$r$ & $699.92 \pm 0.42$ & $15.633 \pm 0.005$ & $\ldots$ & -12.2 & +20.3 & 12 & Spline \\
\hline$i$ & $695.40 \pm 0.05$ & $15.924 \pm 0.006$ & $\ldots$ & -7.8 & +32.7 & 13 & Spline \\
\hline$Y$ & $694.18 \pm 0.10$ & $15.649 \pm 0.009$ & $\ldots$ & -5.5 & +24.0 & 18 & Spline \\
\hline$J$ & $694.56 \pm 0.11$ & $15.494 \pm 0.009$ & $\ldots$ & -5.9 & +23.6 & 16 & Spline \\
\hline \multirow[t]{2}{*}{$H$} & $693.99 \pm 0.20$ & $15.628 \pm 0.039$ & $\ldots$ & -5.3 & +20.3 & 16 & Spline \\
\hline & & & SN 2005ke & & & & \\
\hline$B$ & $699.16 \pm 0.04$ & $14.882 \pm 0.005$ & $1.273 \pm 0.010$ & -9.4 & +267.4 & 39 & Spline \\
\hline V & $701.57 \pm 0.07$ & $14.185 \pm 0.004$ & $1.657 \pm 0.011$ & -11.8 & +265.0 & 39 & Spline \\
\hline$u$ & $698.05 \pm 0.15$ & $16.148 \pm 0.008$ & $1.885 \pm 0.027$ & -8.3 & +268.5 & 36 & Spline \\
\hline$g$ & $699.92 \pm 0.02$ & $14.499 \pm 0.003$ & $1.639 \pm 0.007$ & -10.2 & +266.7 & 39 & Spline \\
\hline$r$ & $702.20 \pm 0.07$ & $14.062 \pm 0.003$ & $2.076 \pm 0.008$ & -12.4 & +263.3 & 40 & Spline \\
\hline$i$ & $701.80 \pm 0.06$ & $14.317 \pm 0.004$ & $2.021 \pm 0.010$ & -12.0 & +264.8 & 39 & Spline \\
\hline$Y$ & $701.41 \pm 0.17$ & $14.076 \pm 0.007$ & $1.390 \pm 0.018$ & -9.6 & +87.7 & 57 & Spline \\
\hline$J$ & $700.50 \pm 0.06$ & $14.021 \pm 0.007$ & $3.389 \pm 0.021$ & -8.7 & +88.6 & 54 & Spline \\
\hline \multirow[t]{2}{*}{$H$} & $701.76 \pm 0.18$ & $13.951 \pm 0.007$ & $2.253 \pm 0.013$ & -9.9 & +87.4 & 56 & Spline \\
\hline & & & SN 2005ki & & & & \\
\hline$B$ & $705.59 \pm 0.11$ & $15.705 \pm 0.005$ & $0.641 \pm 0.009$ & -9.5 & +152.9 & 43 & Spline \\
\hline V & $706.70 \pm 0.09$ & $15.672 \pm 0.005$ & $1.143 \pm 0.010$ & -10.6 & +151.8 & 43 & Spline \\
\hline$u$ & $702.77 \pm 0.10$ & $16.050 \pm 0.008$ & $1.336 \pm 0.032$ & -6.8 & +80.4 & 31 & Spline \\
\hline$g$ & $706.13 \pm 0.07$ & $15.629 \pm 0.004$ & $1.063 \pm 0.009$ & -10.1 & +152.4 & 47 & Spline \\
\hline$r$ & $707.03 \pm 1.21$ & $15.737 \pm 0.020$ & $1.651 \pm 0.022$ & -11.0 & +151.5 & 47 & Spline \\
\hline$i$ & $701.33 \pm 0.23$ & $16.208 \pm 0.015$ & $1.225 \pm 0.019$ & -5.4 & +157.1 & 47 & Spline \\
\hline Y & $701.75 \pm 0.05$ & $16.230 \pm 0.013$ & $0.278 \pm 0.021$ & -4.8 & +54.0 & 22 & Spline \\
\hline$J$ & $702.08 \pm 1.28$ & $16.163 \pm 0.072$ & $1.921 \pm 0.083$ & -5.1 & +53.7 & 21 & Spline \\
\hline \multirow[t]{2}{*}{$H$} & $702.03 \pm 0.44$ & $16.236 \pm 0.027$ & $1.072 \pm 0.047$ & -4.1 & +53.8 & 17 & Spline \\
\hline & & & SN 2005lu & & & & \\
\hline$B$ & $711.90 \pm 0.45$ & $17.380 \pm 0.018$ & $\ldots$ & +9.0 & +90.5 & 17 & Templ. \\
\hline
\end{tabular}


Table 2-Continued

\begin{tabular}{|c|c|c|c|c|c|c|c|}
\hline \multirow{2}{*}{$\begin{array}{c}\text { Band } \\
(1) \\
V\end{array}$} & \multirow{2}{*}{$\begin{array}{c}t^{\max } \\
-2,453,000 \\
(2)\end{array}$} & \multirow{2}{*}{$\begin{array}{c}\begin{array}{c}m^{\max } \\
(\mathrm{mag}) \\
(3)\end{array} \\
17.163 \pm 0.013\end{array}$} & \multirow{2}{*}{$\begin{array}{c}\begin{array}{c}\Delta m_{t} \\
(\mathrm{mag}) \\
(4)\end{array} \\
\ldots\end{array}$} & \multicolumn{2}{|c|}{$\begin{array}{c}\text { Epoch } \\
\text { Range } \\
(5)\end{array}$} & \multirow{2}{*}{$\begin{array}{c}\text { Npts. } \\
(6)\end{array}$} & \multirow{2}{*}{ 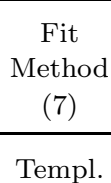 } \\
\hline & & & & +7.6 & +89.1 & & \\
\hline$u$ & $710.36 \pm 0.45$ & $17.920 \pm 0.058$ & $\ldots$ & +10.6 & +28.2 & 5 & Templ. \\
\hline$g$ & $712.35 \pm 0.45$ & $17.225 \pm 0.014$ & $\ldots$ & +8.5 & +50.9 & 10 & Templ. \\
\hline$r$ & $713.73 \pm 0.45$ & $17.135 \pm 0.011$ & $\ldots$ & +7.1 & +88.6 & 16 & Templ. \\
\hline$i$ & $709.23 \pm 0.45$ & $17.659 \pm 0.015$ & $\ldots$ & +11.8 & +93.3 & 16 & Templ. \\
\hline \multicolumn{8}{|c|}{ SN 2005na } \\
\hline$B$ & $740.02 \pm 0.20$ & $16.281 \pm 0.009$ & $0.420 \pm 0.012$ & -1.3 & +92.1 & 26 & Spline \\
\hline V & $741.70 \pm 0.29$ & $16.257 \pm 0.006$ & $0.967 \pm 0.011$ & -2.9 & +90.4 & 27 & Spline \\
\hline$u$ & $738.81 \pm 0.17$ & $16.442 \pm 0.072$ & $1.369 \pm 0.231$ & -0.1 & +47.0 & 17 & Templ. \\
\hline$g$ & $740.20 \pm 0.31$ & $16.235 \pm 0.006$ & $0.740 \pm 0.008$ & -1.5 & +76.3 & 26 & Spline \\
\hline$r$ & $742.25 \pm 0.11$ & $16.306 \pm 0.006$ & $1.238 \pm 0.013$ & -3.5 & +89.9 & 27 & Spline \\
\hline$i$ & $737.51 \pm 0.17$ & $16.754 \pm 0.050$ & $\ldots$ & +1.2 & +99.7 & 27 & Templ. \\
\hline$Y$ & $735.52 \pm 0.17$ & $16.912 \pm 0.017$ & $\ldots$ & +18.6 & +71.9 & 21 & Templ. \\
\hline$J$ & $736.69 \pm 0.17$ & $16.738 \pm 0.041$ & $\ldots$ & +17.4 & +70.7 & 13 & Templ. \\
\hline$H$ & $736.14 \pm 0.17$ & $16.975 \pm 0.047$ & $\ldots$ & +18.0 & +71.3 & 16 & Templ. \\
\hline \multicolumn{8}{|c|}{ SN 2006D } \\
\hline$B$ & $757.95 \pm 0.10$ & $14.321 \pm 0.006$ & $0.773 \pm 0.007$ & -6.1 & +111.8 & 39 & Spline \\
\hline V & $759.24 \pm 0.13$ & $14.180 \pm 0.004$ & $1.226 \pm 0.010$ & -7.3 & +110.5 & 41 & Spline \\
\hline$u$ & $755.54 \pm 0.15$ & $14.994 \pm 0.013$ & $1.564 \pm 0.018$ & -3.7 & +84.4 & 32 & Spline \\
\hline$g$ & $758.20 \pm 0.08$ & $14.225 \pm 0.004$ & $1.097 \pm 0.006$ & -6.3 & +111.5 & 42 & Spline \\
\hline$r$ & $758.60 \pm 0.09$ & $14.185 \pm 0.006$ & $1.670 \pm 0.010$ & -6.7 & +111.1 & 42 & Spline \\
\hline$i$ & $755.23 \pm 0.07$ & $14.595 \pm 0.005$ & $1.420 \pm 0.009$ & -3.3 & +114.5 & 42 & Spline \\
\hline$Y$ & $754.38 \pm 0.05$ & $14.650 \pm 0.050$ & $\ldots$ & -0.5 & +92.1 & 33 & Templ. \\
\hline$J$ & $754.81 \pm 0.05$ & $14.395 \pm 0.050$ & $\ldots$ & -0.9 & +91.7 & 27 & Templ. \\
\hline$H$ & $754.47 \pm 0.06$ & $14.533 \pm 0.050$ & $\ldots$ & -0.7 & +92.0 & 28 & Templ. \\
\hline$K$ & $755.63 \pm 0.05$ & $14.532 \pm 0.058$ & $\ldots$ & -1.8 & +90.9 & 8 & Templ. \\
\hline \multicolumn{8}{|c|}{ SN 2006X } \\
\hline$B$ & $786.15 \pm 0.12$ & $15.321 \pm 0.003$ & $0.579 \pm 0.007$ & -10.4 & +119.7 & 41 & Spline \\
\hline V & $788.91 \pm 0.07$ & $14.090 \pm 0.006$ & $0.938 \pm 0.009$ & -13.2 & +116.9 & 41 & Spline \\
\hline$u$ & $784.55 \pm 0.21$ & $17.373 \pm 0.013$ & $1.113 \pm 0.028$ & -8.8 & +56.9 & 23 & Spline \\
\hline$g$ & $787.79 \pm 0.07$ & $14.771 \pm 0.003$ & $0.805 \pm 0.007$ & -12.1 & +110.2 & 39 & Spline \\
\hline$r$ & $788.77 \pm 0.16$ & $13.643 \pm 0.006$ & $1.263 \pm 0.008$ & -13.0 & +109.2 & 39 & Spline \\
\hline$i$ & $784.54 \pm 0.18$ & $13.836 \pm 0.005$ & $0.799 \pm 0.011$ & -8.8 & +113.4 & 39 & Spline \\
\hline$Y$ & $777.62 \pm 2.06$ & $13.346 \pm 0.059$ & $\ldots$ & -0.8 & +90.5 & 42 & Spline \\
\hline$J$ & $782.77 \pm 0.15$ & $12.824 \pm 0.009$ & $1.557 \pm 0.015$ & -5.9 & +85.4 & 43 & Spline \\
\hline$H$ & $781.69 \pm 0.27$ & $12.995 \pm 0.007$ & $0.444 \pm 0.013$ & -4.8 & +86.5 & 42 & Spline \\
\hline$K$ & $783.95 \pm 0.09$ & $12.790 \pm 0.031$ & $\ldots$ & +0.9 & +63.1 & 18 & Templ. \\
\hline \multicolumn{8}{|c|}{ SN 2006ax } \\
\hline$B$ & $827.40 \pm 0.13$ & $15.202 \pm 0.003$ & $0.493 \pm 0.010$ & -11.6 & +70.0 & 29 & Spline \\
\hline V & $828.51 \pm 0.09$ & $15.213 \pm 0.002$ & $0.927 \pm 0.006$ & -12.7 & +68.9 & 26 & Spline \\
\hline$u$ & $825.70 \pm 0.10$ & $15.545 \pm 0.007$ & $1.346 \pm 0.018$ & -9.9 & +45.1 & 21 & Spline \\
\hline$g$ & $827.65 \pm 0.06$ & $15.147 \pm 0.003$ & $0.789 \pm 0.007$ & -11.8 & +69.7 & 26 & Spline \\
\hline$r$ & $828.74 \pm 0.12$ & $15.314 \pm 0.003$ & $1.137 \pm 0.007$ & -12.9 & +68.6 & 26 & Spline \\
\hline$i$ & $824.06 \pm 0.28$ & $15.822 \pm 0.006$ & $0.745 \pm 0.010$ & -8.3 & +73.2 & 26 & Spline \\
\hline
\end{tabular}


Table 2-Continued

\begin{tabular}{|c|c|c|c|c|c|c|c|}
\hline \multirow{2}{*}{$\begin{array}{l}\text { Band } \\
(1)\end{array}$} & \multirow{2}{*}{$\begin{array}{c}t^{\max } \\
-2,453,000 \\
(2)\end{array}$} & \multirow{2}{*}{$\begin{array}{c}\begin{array}{c}m^{\max } \\
(\mathrm{mag}) \\
(3)\end{array} \\
15.841 \pm 0.008\end{array}$} & \multirow{2}{*}{$\begin{array}{c}\begin{array}{c}\Delta m_{t} \\
(\mathrm{mag}) \\
(4)\end{array} \\
0.043 \pm 0.017\end{array}$} & \multicolumn{2}{|c|}{$\begin{array}{c}\text { Epoch } \\
\text { Range } \\
\quad(5)\end{array}$} & \multirow{2}{*}{$\begin{array}{c}\begin{array}{c}\text { Npts. } \\
(6)\end{array} \\
36\end{array}$} & \multirow{2}{*}{$\begin{array}{c}\begin{array}{c}\text { Fit } \\
\text { Method } \\
(7)\end{array} \\
\text { Spline }\end{array}$} \\
\hline & & & & -5.6 & +45.4 & & \\
\hline$J$ & $823.85 \pm 0.19$ & $15.756 \pm 0.011$ & $1.494 \pm 0.030$ & -6.0 & +45.0 & 33 & Spline \\
\hline$H$ & $823.58 \pm 0.13$ & $15.914 \pm 0.019$ & $0.410 \pm 0.045$ & -5.7 & +45.3 & 25 & Spline \\
\hline$K$ & $824.53 \pm 0.07$ & $15.862 \pm 0.064$ & $\ldots$ & +9.3 & +19.5 & 6 & Templ. \\
\hline \multicolumn{8}{|c|}{ SN 2006bh } \\
\hline$B$ & $833.73 \pm 0.06$ & $14.456 \pm 0.005$ & $0.729 \pm 0.007$ & -4.8 & +59.4 & 25 & Spline \\
\hline V & $834.88 \pm 0.08$ & $14.461 \pm 0.002$ & $1.154 \pm 0.007$ & -5.9 & +58.3 & 26 & Spline \\
\hline$u$ & $831.88 \pm 0.08$ & $14.867 \pm 0.005$ & $1.614 \pm 0.010$ & -2.0 & +61.3 & 21 & Spline \\
\hline$g$ & $834.48 \pm 0.25$ & $14.413 \pm 0.006$ & $1.143 \pm 0.007$ & -5.5 & +58.7 & 24 & Spline \\
\hline$r$ & $835.03 \pm 0.11$ & $14.497 \pm 0.006$ & $1.676 \pm 0.008$ & -6.1 & +58.1 & 26 & Spline \\
\hline$i$ & $830.36 \pm 0.11$ & $14.975 \pm 0.004$ & $1.322 \pm 0.009$ & -1.4 & +62.8 & 25 & Spline \\
\hline$Y$ & $830.12 \pm 0.04$ & $14.881 \pm 0.050$ & $\ldots$ & -0.2 & +39.2 & 24 & Templ. \\
\hline$J$ & $830.56 \pm 0.04$ & $14.801 \pm 0.050$ & $\cdots$ & -0.7 & +38.7 & 21 & Templ. \\
\hline$H$ & $830.26 \pm 0.04$ & $14.977 \pm 0.050$ & $\ldots$ & -0.3 & +39.1 & 19 & Templ. \\
\hline \multicolumn{8}{|c|}{ SN 2006eq } \\
\hline$B$ & $977.11 \pm 0.35$ & $18.593 \pm 0.023$ & $\ldots$ & +4.8 & +49.9 & 14 & Templ. \\
\hline$V$ & $979.02 \pm 0.35$ & $18.328 \pm 0.013$ & $\cdots$ & +2.8 & +53.1 & 16 & Templ. \\
\hline$u$ & $975.45 \pm 0.35$ & $19.339 \pm 0.142$ & $\ldots$ & +6.5 & +29.5 & 6 & Templ. \\
\hline$g$ & $977.80 \pm 0.35$ & $18.451 \pm 0.014$ & $\cdots$ & +4.0 & +54.4 & 17 & Templ. \\
\hline$r$ & $979.08 \pm 0.35$ & $18.241 \pm 0.009$ & $\cdots$ & +2.7 & +66.8 & 18 & Templ. \\
\hline$i$ & $976.88 \pm 0.35$ & $18.653 \pm 0.014$ & $\ldots$ & +5.0 & +69.1 & 18 & Templ. \\
\hline$Y$ & $975.41 \pm 0.35$ & $18.455 \pm 0.025$ & $\cdots$ & +5.4 & +43.2 & 12 & Templ. \\
\hline$J$ & $976.21 \pm 0.35$ & $18.459 \pm 0.083$ & $\ldots$ & +4.6 & +42.4 & 9 & Templ. \\
\hline$H$ & $977.27 \pm 0.35$ & $18.281 \pm 0.138$ & $\ldots$ & +3.5 & +41.3 & 9 & Templ. \\
\hline \multicolumn{8}{|c|}{ SN 2006gt } \\
\hline$B$ & $1004.01 \pm 0.28$ & $18.343 \pm 0.014$ & $\ldots$ & -2.2 & +59.9 & 12 & Spline \\
\hline$V$ & $1005.53 \pm 0.37$ & $18.112 \pm 0.009$ & $\ldots$ & -3.7 & +58.4 & 13 & Spline \\
\hline$u$ & $1001.53 \pm 0.12$ & $19.048 \pm 0.048$ & $\ldots$ & +0.1 & +18.0 & 5 & Templ. \\
\hline$g$ & $1004.63 \pm 0.18$ & $18.183 \pm 0.009$ & $\cdots$ & -2.8 & +59.3 & 12 & Spline \\
\hline$r$ & $1005.65 \pm 0.33$ & $18.153 \pm 0.011$ & $\ldots$ & -3.8 & +58.3 & 13 & Spline \\
\hline$i$ & $1002.49 \pm 2.22$ & $18.464 \pm 0.031$ & $\ldots$ & -0.8 & +61.4 & 13 & Spline \\
\hline$Y$ & $1002.92 \pm 0.12$ & $18.387 \pm 0.024$ & $\ldots$ & -0.3 & +47.9 & 20 & Templ. \\
\hline$J$ & $1003.29 \pm 0.12$ & $18.358 \pm 0.053$ & $\ldots$ & -0.6 & +39.1 & 16 & Templ. \\
\hline$H$ & $1004.08 \pm 0.12$ & $18.236 \pm 0.088$ & $\ldots$ & -1.4 & +38.3 & 11 & Templ. \\
\hline \multicolumn{8}{|c|}{ SN $2006 \mathrm{mr}$} \\
\hline$B$ & $1050.94 \pm 0.12$ & $15.402 \pm 0.014$ & $1.425 \pm 0.029$ & -4.2 & +96.1 & 30 & Spline \\
\hline$V$ & $1052.83 \pm 0.08$ & $14.612 \pm 0.010$ & $1.761 \pm 0.019$ & -6.1 & +94.2 & 31 & Spline \\
\hline$u$ & $1049.46 \pm 0.18$ & $16.616 \pm 0.017$ & $2.068 \pm 0.054$ & -2.8 & +50.9 & 19 & Spline \\
\hline$g$ & $1051.37 \pm 0.11$ & $15.020 \pm 0.011$ & $1.647 \pm 0.017$ & -4.7 & +95.6 & 31 & Spline \\
\hline$r$ & $1053.72 \pm 0.07$ & $14.378 \pm 0.014$ & $2.407 \pm 0.019$ & -7.0 & +93.3 & 32 & Spline \\
\hline$i$ & $1054.59 \pm 0.17$ & $14.503 \pm 0.009$ & $2.328 \pm 0.028$ & -7.9 & +92.4 & 31 & Spline \\
\hline$Y$ & $1056.45 \pm 0.19$ & $14.014 \pm 0.007$ & $2.404 \pm 0.022$ & -8.7 & +80.7 & 29 & Spline \\
\hline$J$ & $1054.12 \pm 0.12$ & $14.056 \pm 0.007$ & $3.486 \pm 0.119$ & -6.4 & +83.0 & 19 & Spline \\
\hline$H$ & $1055.31 \pm 0.07$ & $13.850 \pm 0.006$ & $3.299 \pm 0.081$ & -7.6 & +81.8 & 27 & Spline \\
\hline
\end{tabular}


Table 2-Continued

\begin{tabular}{|c|c|c|c|c|c|c|c|}
\hline $\begin{array}{c}\text { Band } \\
\text { (1) }\end{array}$ & $\begin{array}{c}t^{\max } \\
-2,453,000 \\
(2)\end{array}$ & $\begin{array}{c}m^{\max } \\
(\mathrm{mag}) \\
(3)\end{array}$ & $\begin{array}{c}\Delta m_{t} \\
(\mathrm{mag}) \\
(4)\end{array}$ & & $\begin{array}{l}\text { och } \\
\text { nge } \\
5)\end{array}$ & $\begin{array}{c}\text { Npts. } \\
(6)\end{array}$ & $\begin{array}{l}\text { Fit } \\
\text { Method } \\
(7)\end{array}$ \\
\hline \multicolumn{8}{|c|}{ SN 2006py } \\
\hline$B$ & $1070.57 \pm 0.32$ & $18.140 \pm 0.016$ & $\cdots$ & -1.1 & +22.2 & 7 & Templ. \\
\hline$V$ & $1072.16 \pm 0.32$ & $18.019 \pm 0.013$ & $\ldots$ & -2.8 & +20.6 & 7 & Templ. \\
\hline$u$ & $1069.35 \pm 0.32$ & $18.594 \pm 0.052$ & $\cdots$ & +0.2 & +19.3 & 5 & Templ. \\
\hline$g$ & $1071.16 \pm 0.32$ & $18.050 \pm 0.010$ & $\ldots$ & -1.7 & +17.4 & 6 & Templ. \\
\hline$r$ & $1072.56 \pm 0.32$ & $18.058 \pm 0.009$ & $\ldots$ & -3.2 & +20.1 & 7 & Templ. \\
\hline$i$ & $1068.11 \pm 0.32$ & $18.556 \pm 0.018$ & $\ldots$ & +1.5 & +24.9 & 7 & Templ. \\
\hline
\end{tabular}

Note. - SNe with names in bold face belong to the "best-observed" group (see text). Columns: (1) Filter name; (2) Date of maximum; (3) Apparent peak magnitude (Kcorrected); (4) Decline rate measured as the difference in magnitudes at an epoch $t$ and maximum light in each band $(t=10 \mathrm{~d}$ for $B ; t=15 \mathrm{~d}$ for $u g ; t=20 \mathrm{~d}$ for $V ; t=30 \mathrm{~d}$ for $r ; t=35 \mathrm{~d}$ for $i Y$; $t=40$ for $J H$ ); (5) Epochs covered by data in rest-frame days with respect to the time of maximum; (6) Number of data points; (7) Fitting method (spline or template; see text) 
Table 3. Pseudocolors versus $\Delta m_{15}(B)$ relation parameters

\begin{tabular}{ccccc}
\hline \hline $\begin{array}{c}\text { Pseudocolor } \\
(1)\end{array}$ & $\begin{array}{c}a \\
(2)\end{array}$ & $\begin{array}{c}b \\
(3)\end{array}$ & $\begin{array}{c}\text { rms } \\
(4)\end{array}$ & $\begin{array}{c}N_{\mathrm{SNe}} \\
(5)\end{array}$ \\
\hline$u^{\max }-V^{\max }$ & $0.30 \pm 0.04$ & $0.59 \pm 0.25$ & 0.14 & 13 \\
$B^{\max }-V^{\max }$ & $-0.02 \pm 0.01$ & $0.12 \pm 0.05$ & 0.06 & 14 \\
$g^{\max }-r^{\max }$ & $-0.14 \pm 0.02$ & $0.24 \pm 0.06$ & 0.06 & 14 \\
$B^{\max }-r^{\max }$ & $-0.09 \pm 0.02$ & $0.29 \pm 0.07$ & 0.07 & 14 \\
$V^{\max }-i^{\max }$ & $-0.61 \pm 0.02$ & $0.27 \pm 0.08$ & 0.09 & 14 \\
$V^{\max }-Y^{\max }$ & $-0.67 \pm 0.03$ & $0.51 \pm 0.17$ & 0.14 & 13 \\
$V^{\max }-J^{\max }$ & $-0.63 \pm 0.02$ & $0.55 \pm 0.10$ & 0.09 & 14 \\
$V^{\max }-H^{\max }$ & $-0.77 \pm 0.03$ & $0.54 \pm 0.11$ & 0.14 & 14 \\
$V^{\max }-K^{\max }$ & $-0.59 \pm 0.05$ & $0.14 \pm 0.18$ & 0.14 & 8 \\
\hline
\end{tabular}

Note. - Fits of the type: Pseudocolor $=a+b\left[\Delta m_{15}(B)-\right.$ 1.1] valid over the range $0.85<\Delta m_{15}(B)<1.76 \mathrm{mag}$. Columns: (1) Pseudocolor name; (2) Fit intercept; (3) Fit slope; (4) rms of fit in magnitudes; (5) Number of SNe used in fit. 
Table 4. Host-galaxy color excesses from maximum light colors

\begin{tabular}{|c|c|c|c|c|c|c|c|c|c|c|}
\hline SN & $\begin{array}{c}E(B-V) \\
\quad \text { Tail } \\
(2)\end{array}$ & $\begin{array}{c}E(B-V) \\
\operatorname{Max} \\
(3)\end{array}$ & $\begin{array}{c}E(u-V) \\
\operatorname{Max} \\
(4)\end{array}$ & $\begin{array}{c}E(g-r) \\
\operatorname{Max} \\
(5)\end{array}$ & $\begin{array}{c}E(B-r) \\
\operatorname{Max} \\
(6)\end{array}$ & $\begin{array}{c}E(V-i) \\
\quad \operatorname{Max} \\
(7)\end{array}$ & $\begin{array}{c}E(V-Y) \\
\operatorname{Max} \\
(8)\end{array}$ & $\begin{array}{c}E(V-J) \\
\operatorname{Max} \\
(9)\end{array}$ & $\begin{array}{c}E(V-H) \\
\operatorname{Max} \\
(10)\end{array}$ & $\begin{array}{c}E(V-K) \\
\quad \operatorname{Max} \\
(11)\end{array}$ \\
\hline $2004 \mathrm{ef}$ & $0.125(017)$ & $0.089(006)$ & $0.343(014)$ & $0.117(005)$ & $0.137(007)$ & $-0.037(006)$ & $0.117(034)$ & $0.024(080)$ & & $-0.176(473)$ \\
\hline $2004 \mathrm{eo}$ & $0.082(018)$ & $0.020(016)$ & $0.070(026)$ & $-0.023(018)$ & $0.006(015)$ & $-0.004(018)$ & $-0.244(021)$ & $0.054(021)$ & $-0.093(022)$ & $0.013(017)$ \\
\hline 2004 ey & $0.039(005)$ & $-0.061(008)$ & $0.006(015)$ & $-0.004(006)$ & $-0.043(008)$ & $-0.054(008)$ & $-0.111(020)$ & $0.089(051)$ & $-0.055(024)$ & $-0.159(046)$ \\
\hline $2004 \mathrm{gc}$ & $0.200(021)$ & $0.149(037)$ & & $0.206(038)$ & $0.235(041)$ & $0.078(038)$ & & & & \\
\hline $2004 \mathrm{gs}$ & $0.080(010)$ & $0.121(007)$ & $0.300(020)$ & $0.092(007)$ & $0.138(008)$ & $0.080(009)$ & & $0.063(019)$ & & \\
\hline 2004gu & $0.257(017)$ & $0.200(024)$ & $0.549(045)$ & $0.263(013)$ & $0.358(025)$ & $0.128(019)$ & $\ldots$ & & $\ldots$ & $\ldots$ \\
\hline $2005 \mathrm{~A}$ & & $1.005(011)$ & $2.309(047)$ & $1.078(013)$ & $1.451(014)$ & $0.611(018)$ & $1.414(031)$ & $1.540(031)$ & $1.483(039)$ & \\
\hline $2005 \mathrm{M}$ & $0.091(007)$ & $0.050(005)$ & $0.144(019)$ & $0.028(008)$ & $0.067(008)$ & $0.082(006)$ & $0.137(015)$ & $0.210(048)$ & $0.115(020)$ & $0.144(036)$ \\
\hline $2005 \mathrm{~W}$ & & $0.352(011)$ & $0.326(021)$ & $0.182(015)$ & $0.428(013)$ & $0.114(015)$ & & ) & & $\ldots$ \\
\hline $2005 \mathrm{ag}$ & $0.064(026)$ & $0.050(009)$ & & $0.076(007)$ & $0.069(008)$ & $0.227(021)$ & $-0.065(022)$ & $-0.041(038)$ & $0.272(056)$ & \\
\hline $2005 \mathrm{al}$ & $-0.050(004)$ & $-0.045(009)$ & $-0.008(025)$ & $-0.037(012)$ & $-0.056(014)$ & $-0.135(014)$ & $-0.101(023)$ & $-0.020(025)$ & $-0.174(026)$ & $-0.227(064)$ \\
\hline $2005 \mathrm{am}$ & $0.050(005)$ & $0.014(005)$ & $0.034(011)$ & $0.027(007)$ & $0.023(007)$ & $-0.046(011)$ & & $0.044(017)$ & $0.046(014)$ & $0.007(043)$ \\
\hline 2005be & & $-0.012(047)$ & $-0.186(069)$ & $-0.091(047)$ & $-0.092(050)$ & $-0.183(042)$ & .. & & & \\
\hline $2005 \mathrm{bg}$ & $-0.005(028)$ & $0.063(062)$ & $0.152(074)$ & $0.040(066)$ & $-0.000(066)$ & $-0.137(071)$ & $\ldots$ & $\ldots$ & $\ldots$ & $\ldots$ \\
\hline 2005 bo & $0.132(110)$ & $0.277(020)$ & $0.455(060)$ & $0.286(023)$ & $0.360(026)$ & $0.232(038)$ & $\ldots$ & $\ldots$ & $\ldots$ & $\ldots$ \\
\hline $2005 \mathrm{el}$ & $-0.050(008)$ & $-0.064(010)$ & $-0.168(011)$ & $-0.153(014)$ & $-0.121(013)$ & $-0.044(009)$ & $0.071(013)$ & $-0.085(012)$ & $-0.039(013)$ & $-0.096(135)$ \\
\hline $2005 \mathrm{eq}$ & $0.099(012)$ & $0.126(009)$ & $0.263(018)$ & $0.163(009)$ & $0.205(010)$ & $0.054(051)$ & $0.267(051)$ & $0.247(031)$ & $0.142(041)$ & $0.274(073)$ \\
\hline $2005 \mathrm{hc}$ & $0.078(017)$ & $0.035(007)$ & $0.245(013)$ & $0.059(008)$ & $0.070(007)$ & $-0.105(011)$ & $0.333(051)$ & $0.140(047)$ & $0.205(103)$ & \\
\hline $2005 i q$ & $0.028(023)$ & $-0.033(012)$ & $-0.063(016)$ & $-0.069(010)$ & $-0.072(011)$ & $0.001(015)$ & $0.204(051)$ & $-0.030(047)$ & $0.142(051)$ & $\ldots$ \\
\hline 2005 ir & $-0.010(165)$ & $0.097(038)$ & $0.209(087)$ & $0.135(036)$ & $0.107(044)$ & $-0.110(066)$ & & & & \\
\hline $2005 \mathrm{kc}$ & & $0.172(008)$ & $0.422(014)$ & $0.239(008)$ & $0.295(008)$ & $0.273(009)$ & $0.482(013)$ & $0.552(013)$ & $0.515(040)$ & $\ldots$ \\
\hline $2005 \mathrm{ke}$ & $0.036(005)$ & & & & & & & & & \\
\hline $2005 \mathrm{ki}$ & $-0.048(005)$ & $-0.003(007)$ & $-0.074(015)$ & $-0.043(021)$ & $-0.031(021)$ & $-0.006(017)$ & $-0.037(017)$ & $-0.030(073)$ & $0.027(030)$ & $\ldots$ \\
\hline $20051 \mathrm{u}$ & $0.217(021)$ & $0.234(023)$ & $0.544(063)$ & $0.257(020)$ & $0.367(023)$ & $0.147(022)$ & & $\ldots$ & $\ldots$ & $\ldots$ \\
\hline 2005 na & $0.113(011)$ & $-0.017(011)$ & $-0.152(074)$ & $0.022(010)$ & $0.004(013)$ & $0.071(051)$ & & & & \\
\hline $2006 \mathrm{D}$ & $0.014(004)$ & $0.074(007)$ & $0.252(015)$ & $0.054(008)$ & $0.074(009)$ & $0.060(007)$ & $-0.051(050)$ & $0.134(051)$ & $0.122(051)$ & $0.063(058)$ \\
\hline $2006 \mathrm{X}$ & & $1.221(007)$ & $2.943(017)$ & $1.242(008)$ & $1.737(008)$ & $0.838(009)$ & $1.365(060)$ & $1.836(013)$ & $1.796(012)$ & $1.815(032)$ \\
\hline $2006 \mathrm{ax}$ & $-0.011(005)$ & $-0.032(004)$ & $0.013(011)$ & $-0.055(006)$ & $-0.058(006)$ & $-0.023(008)$ & $-0.003(011)$ & $0.028(014)$ & $-0.007(021)$ & $-0.188(064)$ \\
\hline $2006 \mathrm{bh}$ & $-0.009(004)$ & $-0.052(006)$ & $-0.122(009)$ & $-0.048(009)$ & $-0.074(009)$ & $-0.017(006)$ & $0.039(050)$ & $0.055(050)$ & $0.012(050)$ & \\
\hline $2006 \mathrm{eq}$ & & $0.168(027)$ & $0.301(146)$ & $0.162(021)$ & $0.217(029)$ & $0.080(024)$ & $0.163(040)$ & $0.076(089)$ & $0.383(142)$ & $\ldots$ \\
\hline $2006 \mathrm{gt}$ & $-0.021(063)$ & $0.144(017)$ & $0.243(053)$ & $-0.006(016)$ & $0.070(020)$ & $0.065(034)$ & $0.036(031)$ & $-0.014(057)$ & $0.239(090)$ & $\ldots$ \\
\hline $2006 \mathrm{mr}$ & $-0.025(013)$ & & & & & (3) & $\ldots$ & $\ldots$ & $\ldots$ & \\
\hline 2006py & $\ldots$ & $0.085(021)$ & $0.219(056)$ & $0.083(015)$ & $0.109(020)$ & $0.026(024)$ & $\ldots$ & $\ldots$ & $\ldots$ & $\ldots$ \\
\hline
\end{tabular}

Note. - Columns: (1) SN name; (2) Host-galaxy reddening measured from the (B-V) tail (§§.2]; (3)-(11) Host-galaxy reddening measured from pseudocolor at maximum light $(\S 3.3$. 
Table 5. $\quad R_{V}$ values from fits to $E(V-X)_{\max }$ versus $E(B-V)_{\max }$

\begin{tabular}{lccccc}
\hline \hline \multirow{1}{c}{ Sample } & $\begin{array}{c}E(V-i) \\
\operatorname{Max}\end{array}$ & $\begin{array}{c}E(V-Y) \\
\operatorname{Max}\end{array}$ & $\begin{array}{c}E(V-J) \\
\operatorname{Max}\end{array}$ & $\begin{array}{c}E(V-H) \\
\text { Max }\end{array}$ & $\begin{array}{c}\text { Weighted } \\
\text { Average }\end{array}$ \\
\hline Whole & $1.1 \pm 0.2$ & $1.6 \pm 0.2$ & $1.8 \pm 0.1$ & $1.7 \pm 0.1$ & $1.7 \pm 0.1$ \\
Excl. 2005A and 2006X & $3.8 \pm 1.5$ & $3.5 \pm 1.4$ & $3.1 \pm 0.7$ & $3.1 \pm 0.7$ & $3.2 \pm 0.4$ \\
\hline
\end{tabular}


Table 6. Conversion of observed to true color excesses

\begin{tabular}{lc}
\hline \hline Color Excess & Correction Factor $C_{X}$ \\
\hline$E(V-u)$ & 1.0576 \\
$E(V-B)$ & 1.1023 \\
$E(V-g)$ & 1.1855 \\
$E(V-r)$ & 1.2028 \\
$E(V-i)$ & 1.0218 \\
$E(V-Y)$ & 1.0370 \\
$E(V-J)$ & 1.0142 \\
$E(V-H)$ & 0.9951 \\
$E\left(V-K_{s}\right)$ & 0.9818 \\
\hline
\end{tabular}

Note. $-E(V-X)_{\text {true }}=C_{X} * E(V-$ $X)_{\text {observed }}$ 
Table 7. Adopted distances for three SNe with $z<0.01$

\begin{tabular}{|c|c|c|c|c|}
\hline $\mathrm{SN}$ & $\begin{array}{c}\text { Host } \\
\text { Galaxy }\end{array}$ & $\begin{array}{c}\mu \\
(\mathrm{mag})\end{array}$ & $\begin{array}{l}\text { Distance } \\
\text { Method }\end{array}$ & Reference \\
\hline $2005 \mathrm{ke}$ & NGC 1371 & $31.84 \pm 0.08$ & SBF & Tonrv et al. $(2001)^{\mathrm{a}}$ \\
\hline $2006 \mathrm{X}$ & NGC 4321 & $30.91 \pm 0.14$ & Cepheids & Freedman et al. (2001) \\
\hline $2006 \mathrm{mr}$ & NGC 1316 & $31.59 \pm 0.08$ & $\mathrm{SBF}$ & Cantiello et al. (2007) \\
\hline
\end{tabular}


Table 8. Fits of peak magnitudes versus $\Delta m_{15}(B)$ and pseudocolor

\begin{tabular}{|c|c|c|c|c|c|c|c|c|c|c|}
\hline $\begin{array}{l}\text { Fit } \\
\text { No. } \\
(1)\end{array}$ & $\begin{array}{c}\text { Filter } \\
\quad X \\
(2)\end{array}$ & $\begin{array}{l}\text { Pseudocolor } \\
\qquad(Y-Z) \\
(3)\end{array}$ & $\begin{array}{c}M_{X}(0) \\
\quad(4)\end{array}$ & $\begin{array}{l}b_{X} \\
(5)\end{array}$ & $\begin{array}{c}\beta_{X}^{Y Z} \\
(6)\end{array}$ & $\begin{array}{c}R_{V} \\
(\mathrm{CCM}+\mathrm{O}) \\
(7)\end{array}$ & $\begin{array}{c}\sigma_{S N} \\
{[\mathrm{mag}]} \\
(8)\end{array}$ & $\begin{array}{c}\text { RMS } \\
{[\mathrm{mag}]} \\
(9)\end{array}$ & $\begin{array}{c}N_{S N e} \\
(10)\end{array}$ & $\begin{array}{c}\text { Sample } \\
(11)\end{array}$ \\
\hline 1 & $B$ & $(B-V)$ & $-19.10 \pm 0.02$ & $0.64 \pm 0.10$ & $2.81 \pm 0.11$ & $1.52 \pm 0.10$ & 0.14 & 0.17 & 31 & whole \\
\hline 2 & $B$ & $(B-V)$ & $-19.09 \pm 0.02$ & $0.74 \pm 0.10$ & $2.74 \pm 0.11$ & $1.46 \pm 0.09$ & 0.12 & 0.15 & 26 & best-observed \\
\hline 3 & $B$ & $(B-V)$ & $-19.10 \pm 0.01$ & $0.61 \pm 0.11$ & $2.79 \pm 0.12$ & $1.50 \pm 0.10$ & 0.12 & 0.16 & 28 & excluding fast-decliners \\
\hline 4 & $B$ & $(B-V)$ & $-19.09 \pm 0.01$ & $0.74 \pm 0.11$ & $2.74 \pm 0.11$ & $1.46 \pm 0.10$ & 0.09 & 0.14 & 23 & best-observed excl. fast-decliners \\
\hline 5 & $B$ & $(B-V)$ & $-19.07 \pm 0.02$ & $0.81 \pm 0.12$ & $2.24 \pm 0.37$ & $1.03 \pm 0.32$ & 0.09 & 0.14 & 21 & best-observed and $(B-V)<0.4$ \\
\hline 6 & $J$ & $(V-J)$ & $-18.33 \pm 0.03$ & $0.65 \pm 0.10$ & $0.20 \pm 0.07$ & $1.60 \pm 0.32$ & 0.08 & 0.14 & 21 & best-observed \\
\hline 7 & $J$ & $(V-J)$ & $-18.36 \pm 0.03$ & $0.52 \pm 0.09$ & $0.13 \pm 0.06$ & $1.32 \pm 0.22$ & 0.04 & 0.12 & 19 & best-observed excl. fast-decliners \\
\hline 8 & $J$ & $(V-J)$ & $-18.44 \pm 0.14$ & $0.57 \pm 0.14$ & $0.01 \pm 0.24$ & $0.96 \pm 0.59$ & 0.05 & 0.12 & 17 & best-observed and $(V-J)<0.0$ \\
\hline
\end{tabular}

Note. - Fits of the type: $\mu_{X}=m_{X}^{\max }-M_{X}(0)-b_{X}\left[\Delta m_{15}(B)-1.1\right]-\beta_{X}^{Y Z}(Y-Z)$.

Columns: (1) Fit identifier; (2) Filter corresponding to $m_{X}^{\max }$; (3) Color; (4) Absolute magnitude for $\Delta m_{15}(B)=1.1$ and zero $(Y-Z)$ color; (5) Luminositydecline rate slope; (6) Luminosity-color slope; (7) Corresponding parameter $R_{V}$ of the CCM+O reddening law; (8) Resulting intrinsic dispersion of SN data; (8) RMS of fit in magnitudes; (10) Number of SNe used in fit; (11) Sample of SNe used in fit (see text). 
Table 9. Fits of peak magnitudes versus $\Delta m_{15}(B)$ and reddening

\begin{tabular}{|c|c|c|c|c|c|c|c|c|c|c|}
\hline $\begin{array}{l}\text { Fit } \\
\text { No. } \\
(1)\end{array}$ & $\begin{array}{c}\text { Filter } \\
\quad X \\
(2)\end{array}$ & $\begin{array}{l}\text { Color excess } \\
E(Y-Z) \\
\quad(3)\end{array}$ & $\begin{array}{c}M_{X}(0) \\
\quad(4)\end{array}$ & $\begin{array}{l}b_{X} \\
(5)\end{array}$ & $\begin{array}{c}R_{X}^{Y Z} \\
(6)\end{array}$ & $\begin{array}{c}R_{V} \\
(\mathrm{CCM}+\mathrm{O}) \\
(7)\end{array}$ & $\begin{array}{c}\sigma_{S N} \\
{[\mathrm{mag}]} \\
(8)\end{array}$ & $\begin{array}{c}\text { RMS } \\
{[\mathrm{mag}]} \\
(9)\end{array}$ & $\begin{array}{c}N_{S N e} \\
(10)\end{array}$ & $\begin{array}{c}\text { Sample } \\
(11)\end{array}$ \\
\hline 1 & $B$ & $E(B-V)$ & $-19.07 \pm 0.01$ & $1.03 \pm 0.25$ & $\ldots$ & $\ldots$ & 0.19 & 0.19 & 12 & low reddening \\
\hline 2 & $B$ & $E(B-V)$ & $-19.39 \pm 0.02$ & $0.97 \pm 0.41$ & 4.53 & 3.10 & 0.54 & 0.53 & 28 & whole \\
\hline 3 & $B$ & $E(B-V)$ & $-19.15 \pm 0.02$ & $0.94 \pm 0.11$ & $2.79 \pm 0.12$ & $1.50 \pm 0.11$ & 0.13 & 0.16 & 28 & whole \\
\hline 4 & $B$ & $E(B-V)$ & $-19.14 \pm 0.01$ & $1.07 \pm 0.11$ & $2.74 \pm 0.11$ & $1.46 \pm 0.10$ & 0.10 & 0.14 & 23 & best-observed \\
\hline 5 & $B$ & $E(B-V)$ & $-19.14 \pm 0.03$ & $0.94 \pm 0.12$ & $2.75 \pm 0.38$ & $1.46 \pm 0.33$ & 0.13 & 0.16 & 26 & excluding $05 \mathrm{~A}$ and $06 \mathrm{X}$ \\
\hline 6 & $B$ & $E(B-V)$ & $-19.11 \pm 0.02$ & $1.08 \pm 0.11$ & $2.22 \pm 0.37$ & $1.01 \pm 0.31$ & 0.09 & 0.14 & 21 & best-observed excl. 05A and 06X \\
\hline 7 & $V$ & $E(B-V)$ & $-19.12 \pm 0.01$ & $0.95 \pm 0.11$ & $1.74 \pm 0.11$ & $1.46 \pm 0.10$ & 0.09 & 0.14 & 23 & best-observed \\
\hline 8 & $u$ & $E(u-V)$ & $-18.83 \pm 0.02$ & $1.59 \pm 0.12$ & $1.74 \pm 0.05$ & $1.10 \pm 0.07$ & 0.10 & 0.13 & 22 & best-observed \\
\hline 9 & $g$ & $E(g-r)$ & $-19.17 \pm 0.01$ & $1.32 \pm 0.12$ & $2.21 \pm 0.11$ & $1.49 \pm 0.12$ & 0.11 & 0.14 & 23 & best-observed \\
\hline 10 & $r$ & $E(B-r)$ & $-19.03 \pm 0.01$ & $1.02 \pm 0.11$ & $0.89 \pm 0.08$ & $1.44 \pm 0.11$ & 0.10 & 0.13 & 23 & best-observed \\
\hline 11 & $i$ & $E(B-V)$ & $-18.50 \pm 0.02$ & $0.71 \pm 0.13$ & $1.08 \pm 0.12$ & $1.71 \pm 0.13$ & 0.12 & 0.15 & 23 & best-observed \\
\hline 12 & $Y$ & $E(B-V)$ & $-18.46 \pm 0.01$ & $0.44 \pm 0.14$ & $0.54 \pm 0.12$ & $1.78 \pm 0.19$ & 0.10 & 0.15 & 18 & best-observed \\
\hline 13 & $J$ & $E(V-J)$ & $-18.44 \pm 0.01$ & $0.58 \pm 0.09$ & $0.14 \pm 0.06$ & $1.37 \pm 0.23$ & 0.03 & 0.12 & 18 & best-observed \\
\hline 14 & $H$ & $E(V-H)$ & $-18.38 \pm 0.02$ & $0.32 \pm 0.18$ & $0.20 \pm 0.09$ & $2.72 \pm 1.79$ & 0.12 & 0.16 & 16 & best-observed \\
\hline 15 & $K$ & $E(V-K)$ & $-18.43 \pm 0.03$ & $0.81 \pm 0.29$ & $0.15 \pm 0.10$ & $4.11 \pm 8.32$ & 0.10 & 0.17 & 9 & best-observed \\
\hline
\end{tabular}

Note. - Fits of the type: $\mu_{X}=m_{X}^{\max }-M_{X}(0)-b_{X}\left[\Delta m_{15}(B)-1.1\right]-R_{X}^{Y Z} E(Y-Z)$.

Columns: (1) Fit identifier; (2) Filter corresponding to $m_{X}^{\max }$; (3) Color excess; (4) Absolute magnitude for $\Delta m_{15}(B)=1.1$ and no reddening; (5) Luminosity-decline rate slope; (6) Total-to-selective absorption coefficient $R_{X}^{Y Z} \equiv A_{X} / E(Y-Z)$; (7) Corresponding parameter $R_{V}$ of the CCM+O reddening law; (8) Resulting intrinsic dispersion of SN data; (9) RMS of fit in magnitudes; (10) Number of SNe used in fit; (11) Sample of SNe used in fit (see text). 
Table 10. Precision of the SNe Ia as standard candles in the NIR

\begin{tabular}{ccccccl}
\hline \hline $\begin{array}{c}\text { Band } \\
X\end{array}$ & $\begin{array}{c}M_{X}^{\max } \\
\text { (avg.) }\end{array}$ & $\begin{array}{c}\text { Adopted } \\
R_{X}^{Y Z}\end{array}$ & $\begin{array}{c}R_{V} \\
(\mathrm{CCM}+\mathrm{O})\end{array}$ & $\begin{array}{c}\text { RMS } \\
{[\mathrm{mag}]}\end{array}$ & $N_{S N e}$ & \multicolumn{1}{c}{ Sample } \\
\hline$Y$ & $-18.44 \pm 0.07$ & $R_{Y}^{V Y}=0.32$ & 1.7 & 0.24 & 17 & best-observed \\
$Y$ & $-18.47 \pm 0.08$ & $R_{Y}^{V Y}=0.60$ & 3.1 & 0.26 & 15 & excluding 05A and 06X \\
$Y$ & $-18.43 \pm 0.08$ & $R_{Y}^{V Y}=0.0$ & 0.0 & 0.19 & 15 & excluding 05A and 06X \\
& & & & & & \\
$J$ & $-18.43 \pm 0.07$ & $R_{J}^{V J}=0.13$ & 1.3 & 0.18 & 18 & best-observed \\
$J$ & $-18.44 \pm 0.07$ & $R_{J}^{V J}=0.39$ & 3.1 & 0.20 & 16 & excluding 05A and 06X \\
$J$ & $-18.42 \pm 0.07$ & $R_{J}^{V J}=0.0$ & 0.0 & 0.18 & 16 & excluding 05A and 06X \\
& & & & & & \\
$H$ & $-18.42 \pm 0.08$ & $R_{H}^{V H}=0.19$ & 2.4 & 0.19 & 16 & best-observed \\
$H$ & $-18.43 \pm 0.08$ & $R_{H}^{V H}=0.22$ & 3.1 & 0.21 & 14 & excluding 05A and 06X \\
$H$ & $-18.40 \pm 0.08$ & $R_{H}^{V H}=0.0$ & 0.0 & 0.19 & 14 & excluding 05A and 06X \\
& & & & & & \\
$K_{s}$ & $-18.47 \pm 0.12$ & $R_{K}^{V K}=0.12$ & 2.7 & 0.27 & 9 & best-observed \\
$K_{s}$ & $-18.49 \pm 0.13$ & $R_{K}^{V K}=0.13$ & 3.1 & 0.29 & 8 & excluding 06X \\
$K_{s}$ & $-18.49 \pm 0.13$ & $R_{K}^{V K}=0.0$ & 0.0 & 0.27 & 8 & excluding 06X \\
\hline
\end{tabular}


Table 11. Comparison of Absolute Magnitudes in $J H K_{s}$

\begin{tabular}{ccccc}
\hline \hline & \multicolumn{2}{c}{ Maximum } & & $t\left(B_{\max }\right)$ \\
Band & Krisciunas et al. (2004b) & This Paper & Wood-Vasev et al. (2008) & This Paper \\
\hline \multirow{2}{*}{$J$} & $-18.61 \pm 0.03$ & $-18.43 \pm 0.07$ & $-18.29 \pm 0.09$ & $-18.42 \pm 0.02$ \\
$H$ & $-18.28 \pm 0.03$ & $-18.42 \pm 0.08$ & $-18.08 \pm 0.09$ & $-18.23 \pm 0.03$ \\
$K_{s}$ & $-18.44 \pm 0.03$ & $-18.47 \pm 0.12$ & $-18.30 \pm 0.11$ & $-18.30 \pm 0.05$ \\
\hline
\end{tabular}


Table 12. Measurements of $R_{V}$ for Individual SNe Ia

\begin{tabular}{ccccl}
\hline \hline \multicolumn{1}{c}{ SN } & $\Delta m_{15}(B)$ & $E(B-V)$ & $R_{V}$ & \multicolumn{1}{c}{ Reference } \\
\hline $1999 \mathrm{cl}$ & $1.29 \pm 0.08$ & $1.24 \pm 0.07$ & $1.55 \pm 0.08$ & Krisciunas et al. (2006) \\
$2001 \mathrm{el}$ & $1.15 \pm 0.04$ & $0.21 \pm 0.05$ & $2.15 \pm 0.23$ & Krisciunas et al. (2007) \\
$2002 \mathrm{cv}$ & $1.46 \pm 0.17$ & $5.45 \pm 0.28$ & $1.59 \pm 0.07$ & Elias-Rosa et al. (2008) \\
$2003 \mathrm{cg}$ & $1.25 \pm 0.05$ & $1.33 \pm 0.11$ & $1.80 \pm 0.19$ & Elias-Rosa et al. (2006) \\
$2005 \mathrm{~A}$ & $1.34 \pm 0.03$ & $1.11 \pm 0.07$ & $1.68 \pm 0.10$ & This paper \\
$2006 \mathrm{X}$ & $1.31 \pm 0.05$ & $1.42 \pm 0.04$ & $1.48 \pm 0.06$ & Wang et al. (2008a) \\
& $1.29 \pm 0.05$ & $1.34 \pm 0.07$ & $1.55 \pm 0.07$ & This paper \\
\hline
\end{tabular}

Note. - The values $\Delta m_{15}(B)$ given here have been corrected for reddening using equation (6) of Phillips et al. (1999). 
Table 13. Reddening-law coefficients for a SN Ia at maximum

\begin{tabular}{lcccccc}
\hline \hline & \multicolumn{2}{c}{$\mathrm{CCM}$} & \multicolumn{2}{c}{$\mathrm{CCM}+\mathrm{O}$} & \multicolumn{2}{c}{$\mathrm{CCM}+\mathrm{O}(\mathrm{Obs})}$. \\
Filter & $a_{X}$ & $b_{X}$ & $a_{X}$ & $b_{X}$ & $a_{X}$ & $b_{X}$ \\
\hline$u$ & 0.990 & 1.605 & 1.006 & 1.480 & 0.959 & 1.849 \\
$B$ & 1.016 & 0.860 & 1.026 & 0.799 & 1 & 1 \\
$g$ & 1.017 & 0.540 & 1.020 & 0.525 & 1.003 & 0.657 \\
$V$ & 1 & 0 & 1 & 0 & 1 & 0 \\
$r$ & 0.939 & -0.245 & 0.942 & -0.284 & 0.952 & -0.356 \\
$i$ & 0.807 & -0.499 & 0.797 & -0.449 & 0.813 & -0.564 \\
$Y$ & 0.539 & -0.502 & 0.538 & -0.503 & 0.557 & -0.636 \\
$J$ & 0.398 & -0.370 & 0.398 & -0.371 & 0.412 & -0.469 \\
$H$ & 0.259 & -0.243 & 0.259 & -0.244 & 0.268 & -0.308 \\
$K$ & 0.166 & -0.153 & 0.165 & -0.153 & 0.171 & -0.193 \\
\hline
\end{tabular}




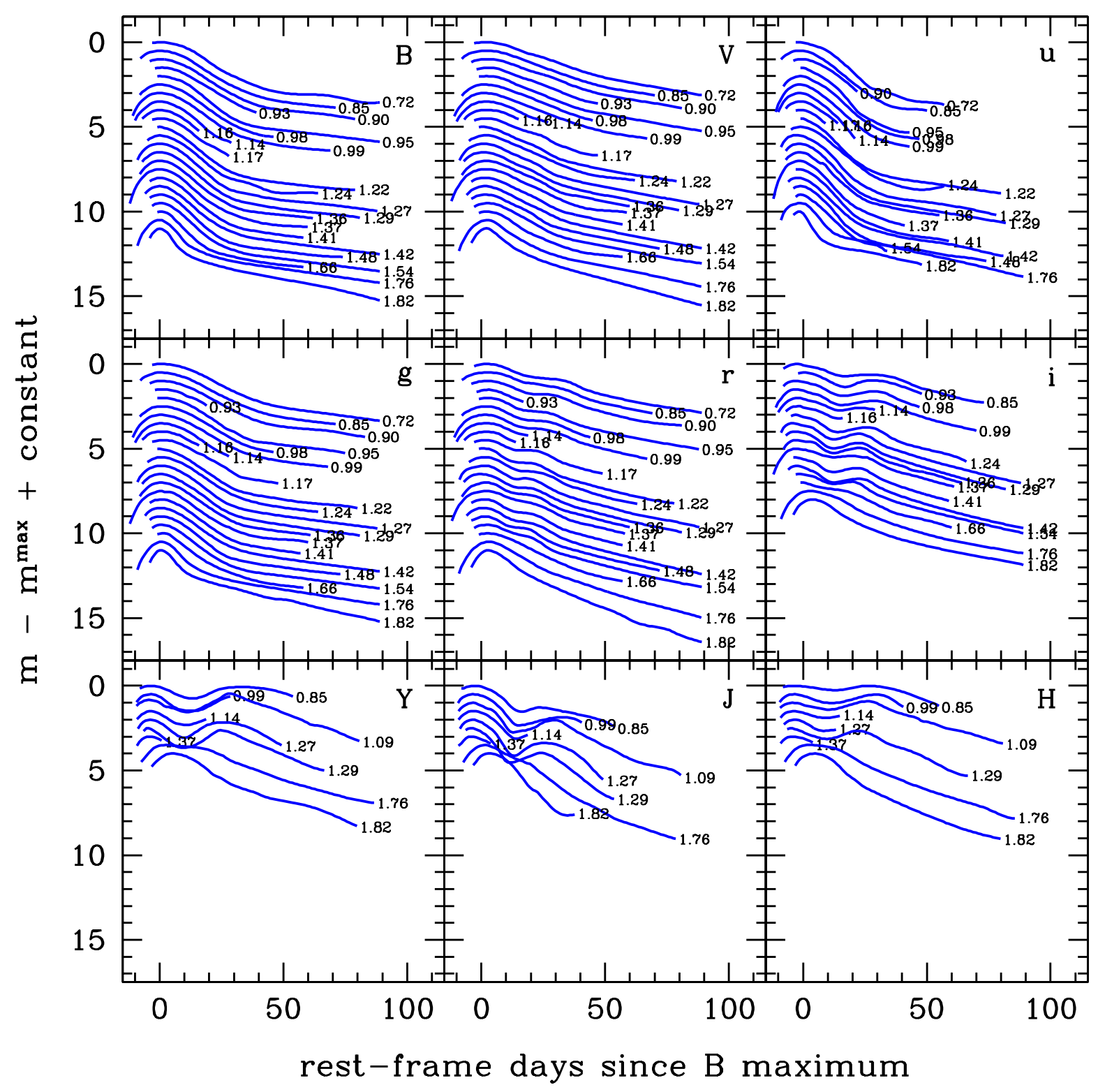

Fig. 1. - Spline fits to the ugriBVYJH light curves of the SNe used as templates. The light curves are sorted by $\Delta m_{15}(B)$ and are plotted relative to the magnitude at maximum light in each band, shifted by $0.5 \mathrm{mag}$ with respect to each other. The values of $\Delta m_{15}(B)$ label each curve at the right end. 


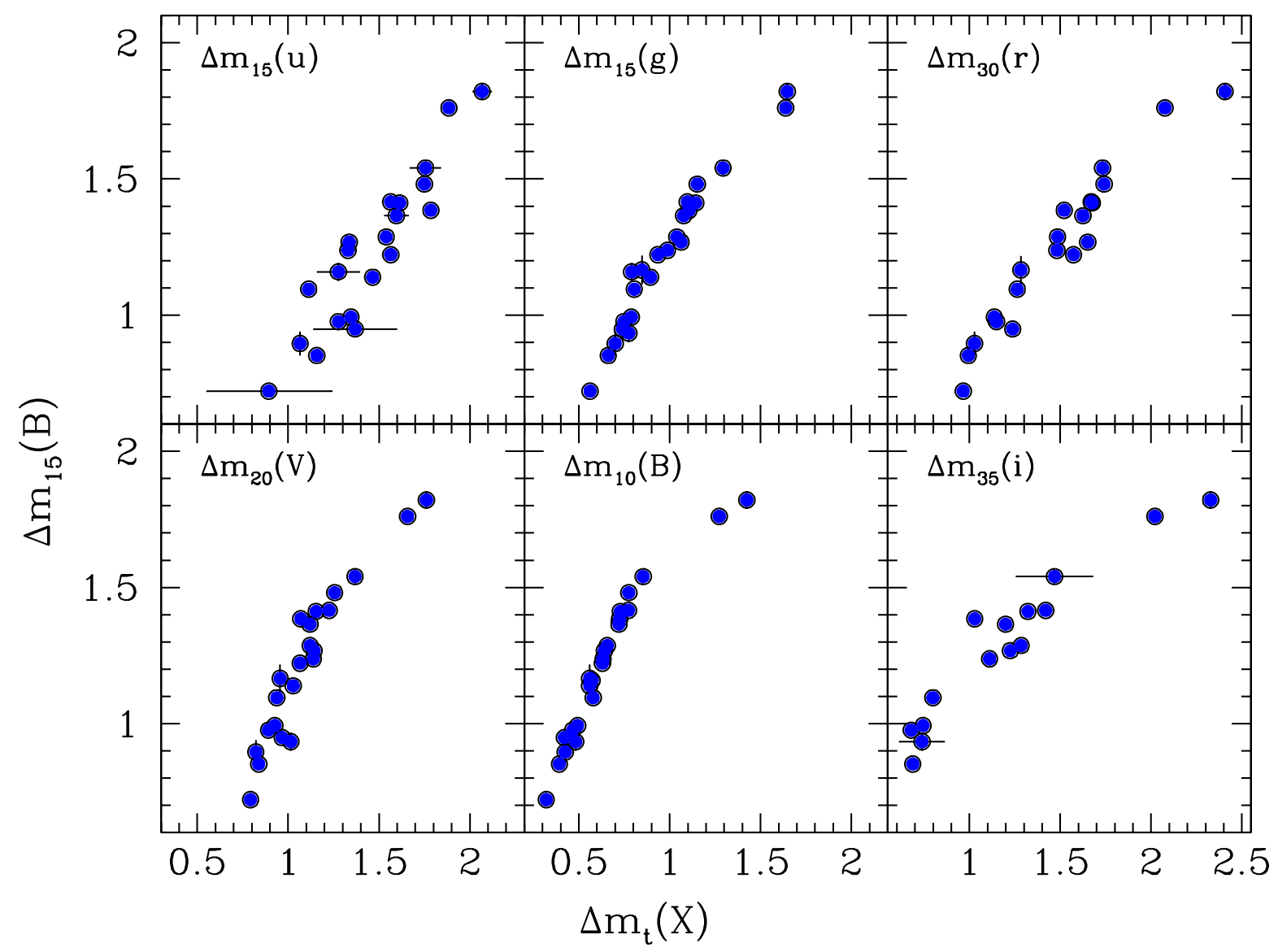

Fig. 2.- Comparison of decline rates $\Delta m_{15}(B)$ with similar quantities obtained from other optical bands and epochs since maximum light. Decline rates $\Delta m_{t}(X)$ are measured from the light curve in $X$ as the difference in magnitudes between $t$ rest-frame days since maximum light (in that band) and the peak magnitude. Only results of spline-function fits to SNe with pre-maximum coverage are shown for each band. Except where plotted, error bars are smaller than the points. 


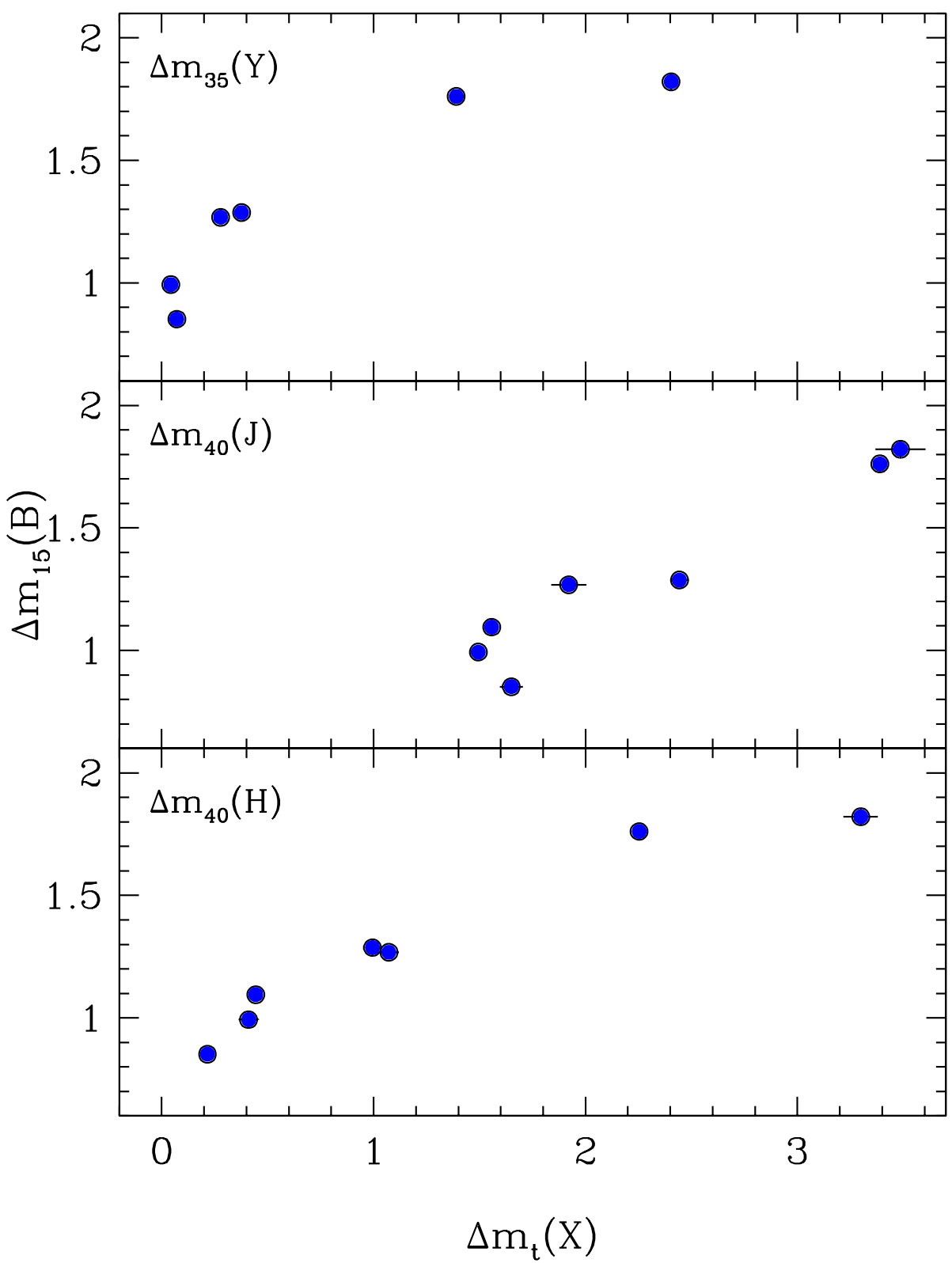

Fig. 3.- Comparison of decline rates $\Delta m_{15}(B)$ with similar quantities obtained from $Y J H$ bands. Decline rates $\Delta m_{t}(X)$ are measured from the light curve in $X$ as the difference in magnitudes between $t$ rest-frame days since maximum light (in that band) and the peak magnitude. Only results of spline-function fits to SNe with pre-maximum coverage are shown for each band. Except where plotted, error bars are smaller than the points. 


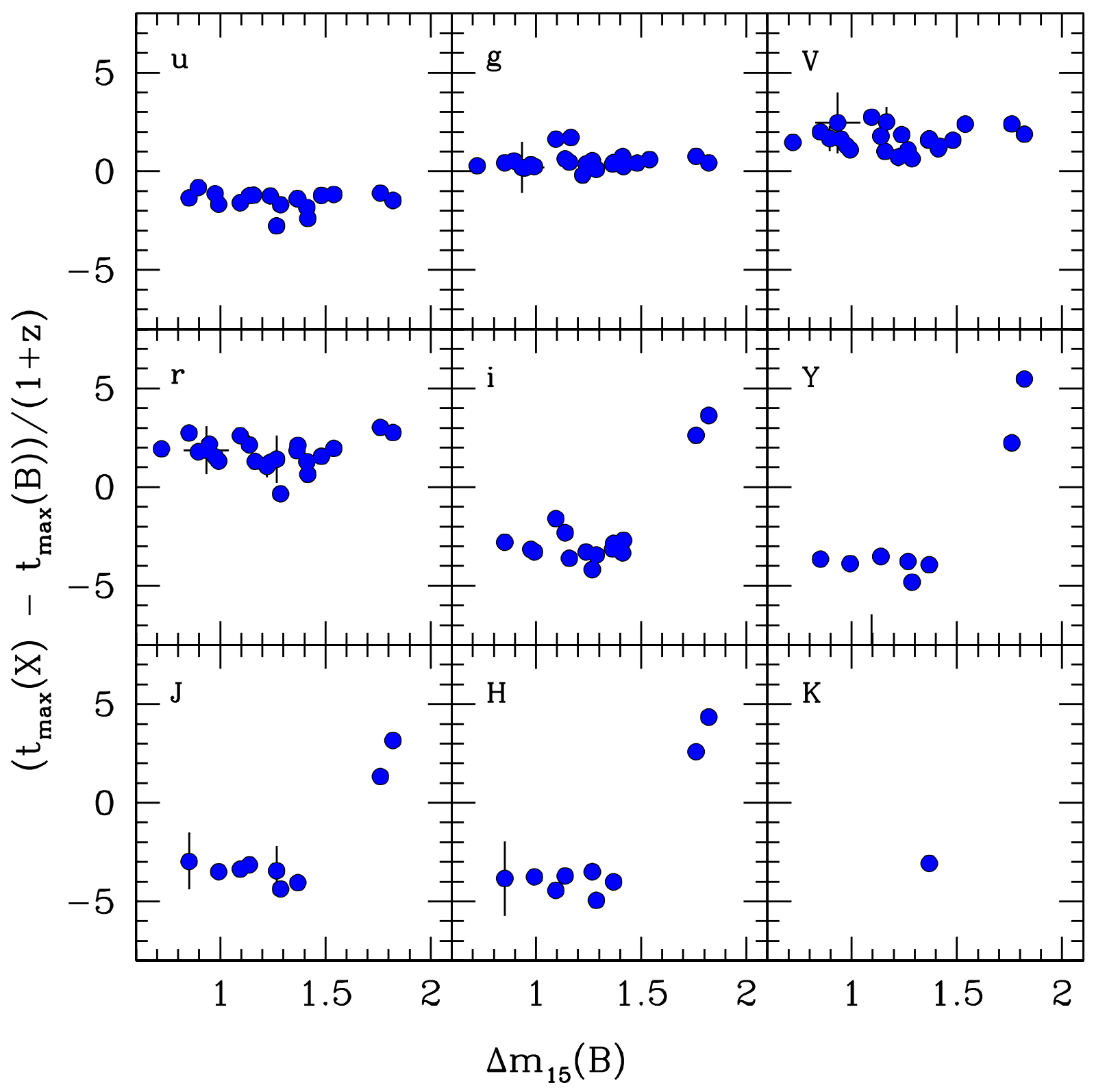

Fig. 4.- Differences in rest-frame days between the time of maximum in ugriVYJHK $K_{s}$ with respect to $B$. Only results of spline-function fits to $\mathrm{SNe}$ with pre-maximum coverage are shown for each band. 

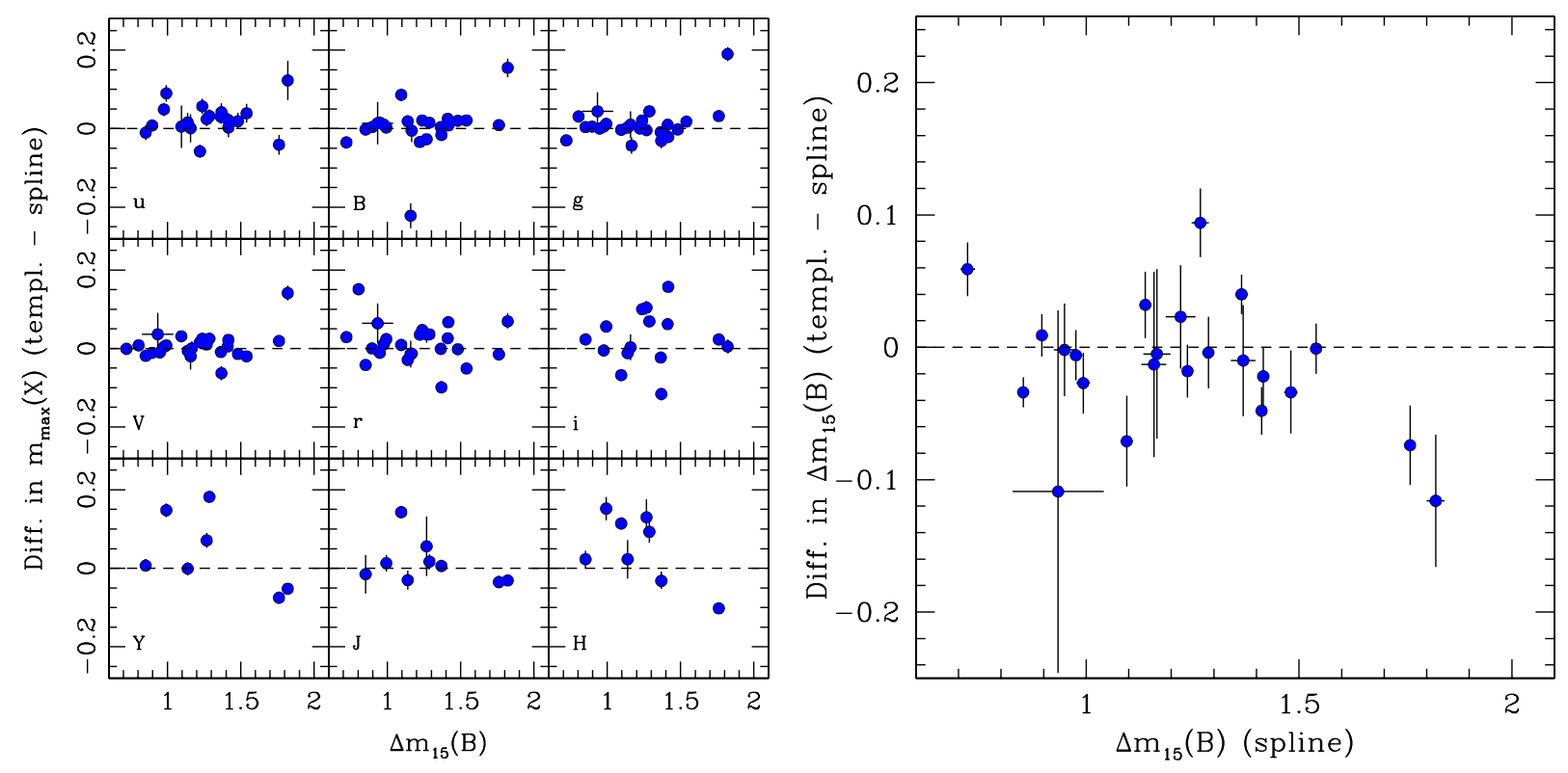

Fig. 5. - (left) Plot of the difference in the peak magnitudes derived from template versus spline fits as a function of filter for the subset of best-observed SNe. (right) Difference in $\Delta m_{15}(B)$ derived from template versus spline fits for the same sample. 


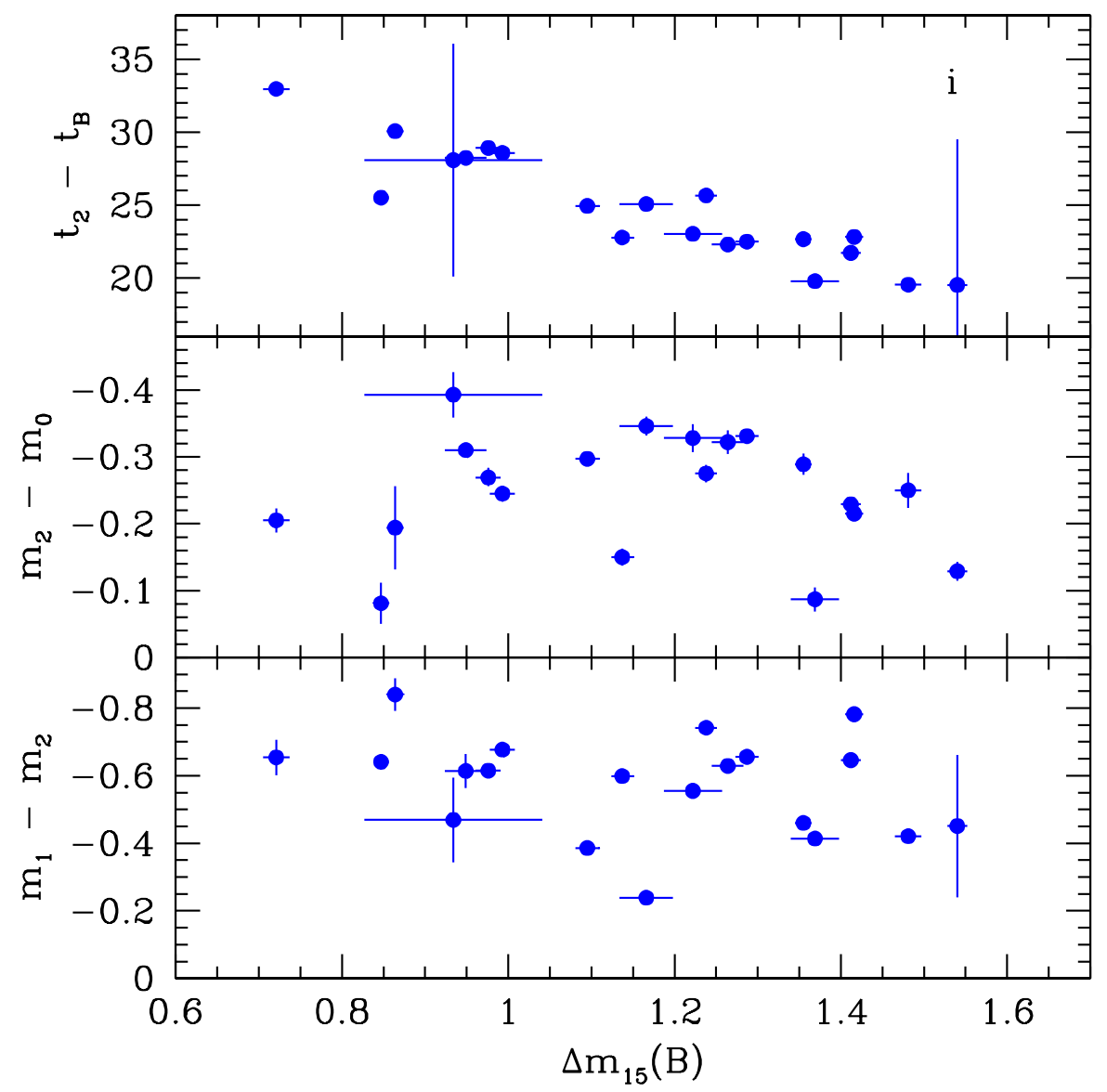

Fig. 6. - Timing and strength of the secondary maximum in $i$ as a function of the decline-rate parameter $\Delta m_{15}(B)$ for the sample of best-observed SNe with pre-maximum data in each band. The top panel shows the phase of the secondary maximum with respect to the $B$-band maximum. The middle panel displays the difference in magnitudes between the secondary maximum and the local minimum between the primary and secondary maxima. The bottom panel shows the difference in magnitude between the primary and secondary maxima. 


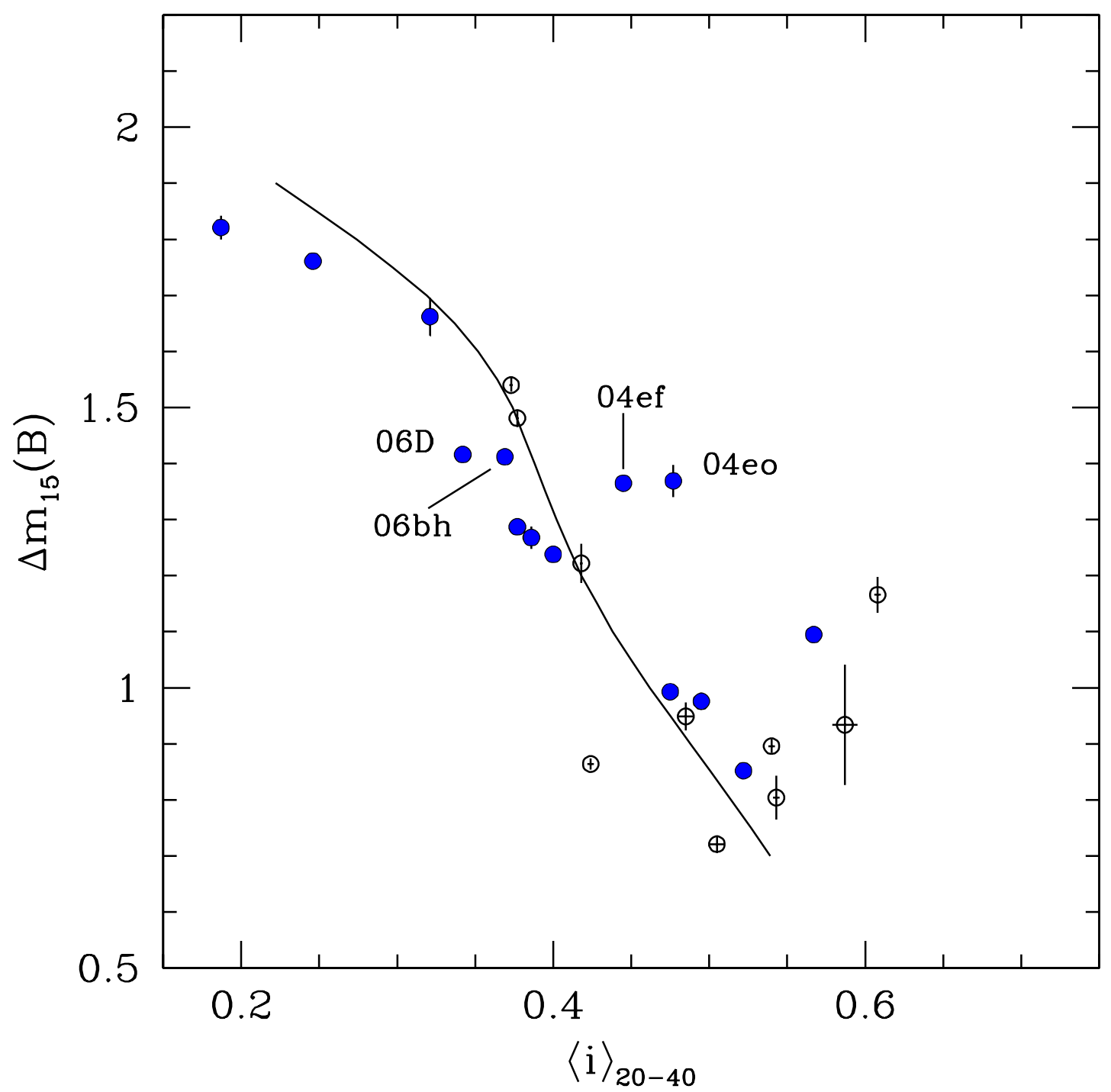

Fig. 7.- The mean $i$-band flux 20 to 40 days after the time of $B$-band maximum plotted versus $\Delta m_{15}(B)$ for the best-observed subsample of CSP SNe. Solid symbols correspond to SNe with peak magnitudes measured from spline fits; open symbols are SNe with peak magnitudes derived from template fits. The solid line corresponds to the relation for the SNOOPy templates. Four SNe with similar $\Delta m_{15}(B)$ but discrepant average $i$-band fluxes at the second maximum are labeled. 

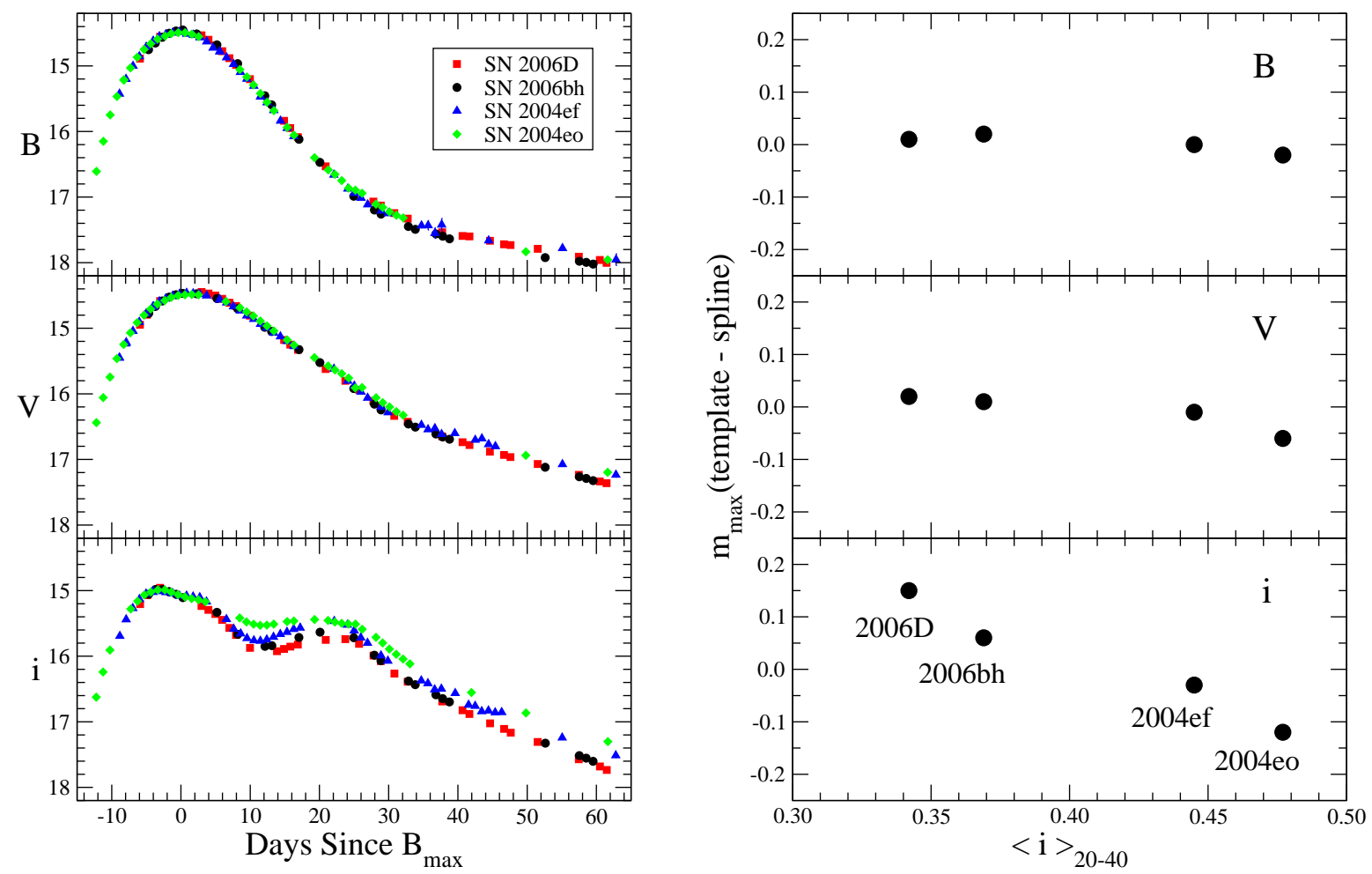

Fig. 8.- (left) Comparison of the $B, V$, and $i$ light curves of SNe 2006D, 2006bh, 2004eo, and 2004ef. The data for SNe 2006D, 2004eo, and 2004ef have been shifted to coincide at maximum with the observations of SN 2006bh. All four SNe have very similar decline rates in the range $\Delta m_{15}(B)=1.37-1.42$ mag. Note the close similarity of the $B$ and $V$ light curves, but the real differences in the strength and morphology of the secondary maxima in the $i$-band. (right) The difference between the maximum-light magnitudes in the $B V i$ bands as measured from template and spline fits for SNe 2006D, 2006bh, 2004eo, and 2004ef, plotted as a function of the mean $i$-band flux 20 to 40 days after the time of $B$-band maximum. 


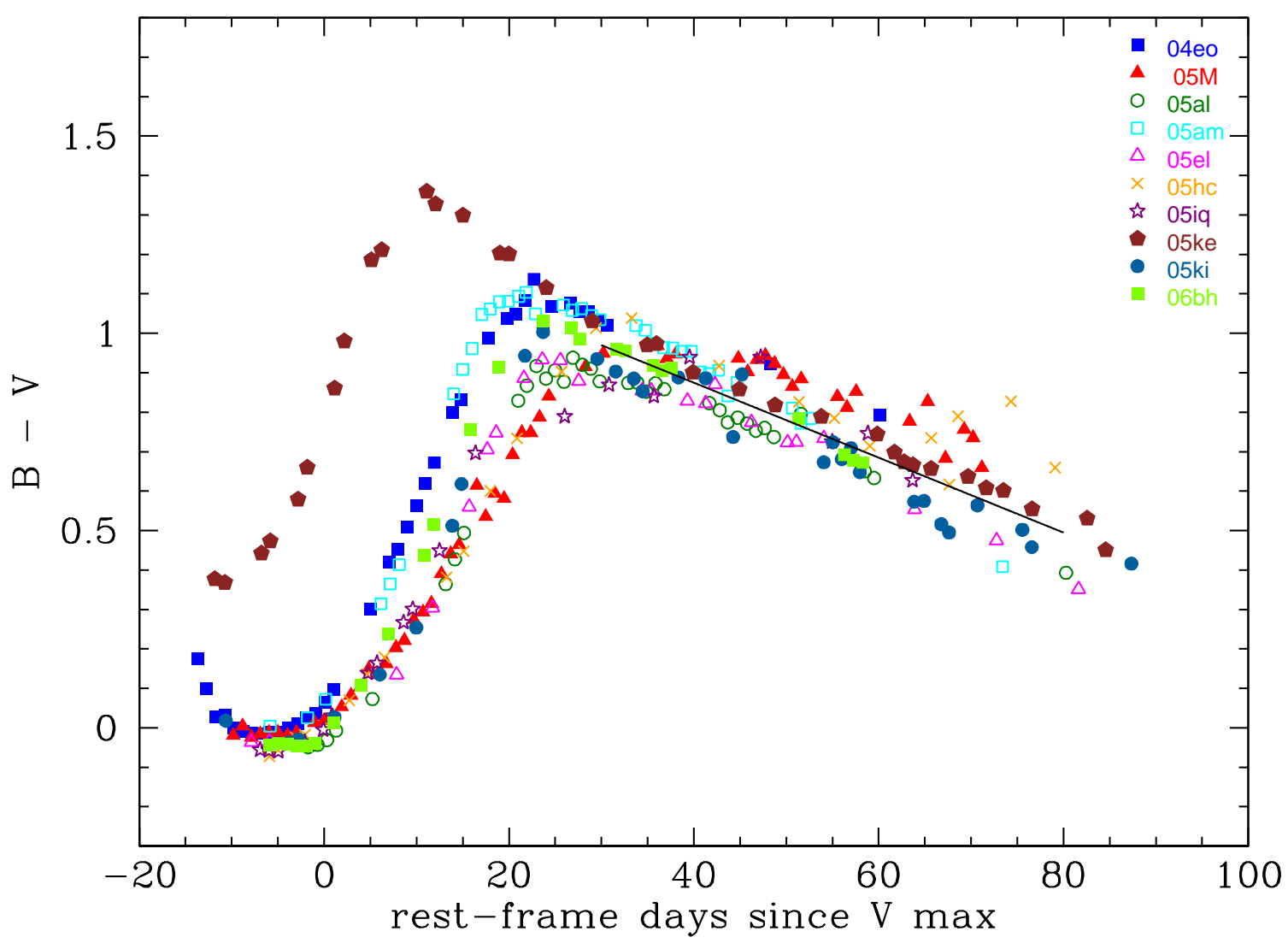

Fig. 9. - Late-time $B-V$ color evolution of SNe assumed to have low or no host-galaxy reddening. Observed colors were corrected for Galactic reddening. The solid line shows a linear fit to the data in the range $30<t_{V}<80$ days (see text). The rms scatter about this fit is 0.077 mag. 


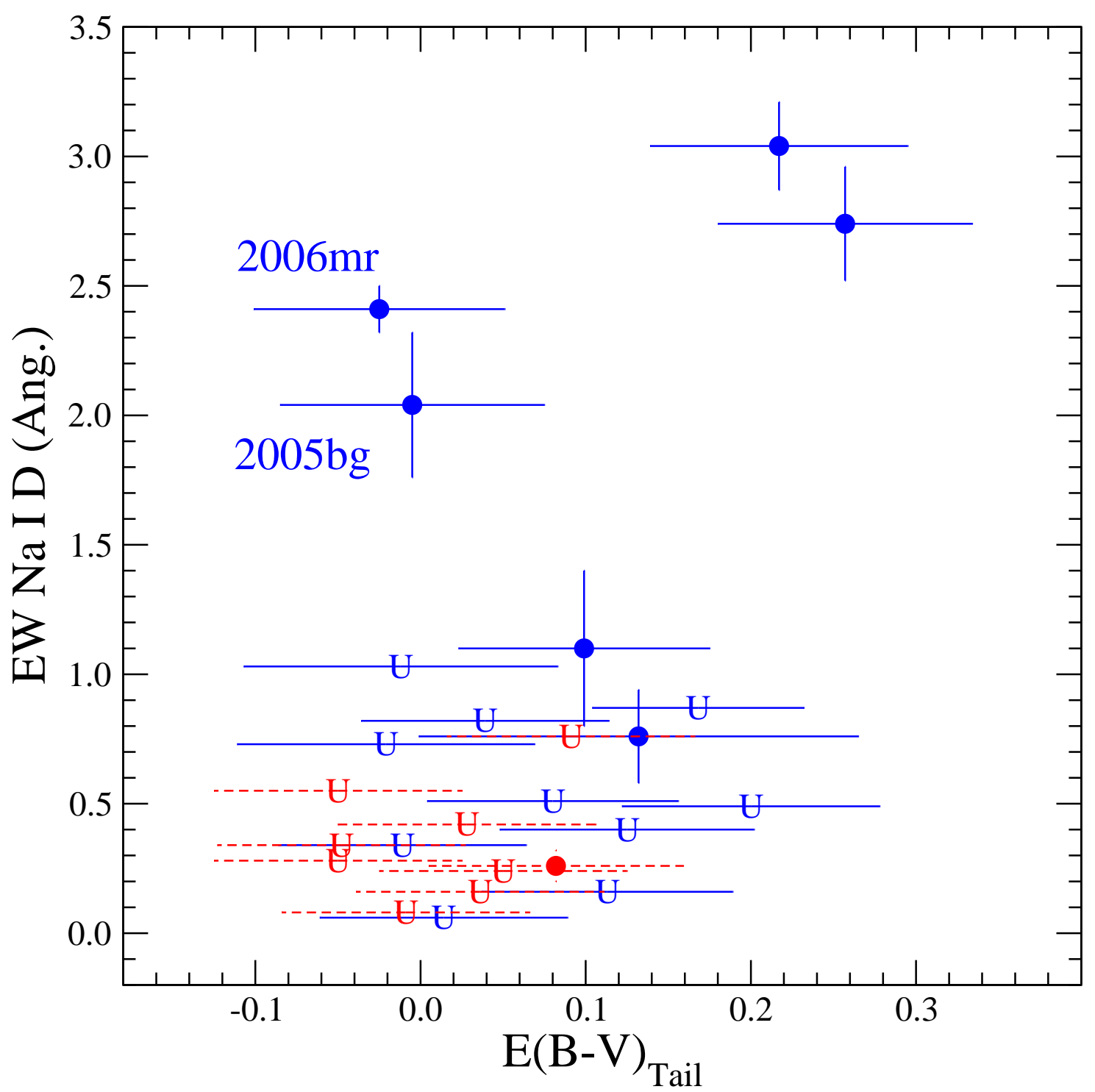

Fig. 10.- Equivalent-width measurements (or upper limits) of the Na I D lines plotted versus the color excess in $B-V$ as measured from the Lira (1995) law. The rms dispersion of $0.077 \mathrm{mag}$ in the Lira-law fit (equation 2) has been added in quadrature to the errors in $E(B-V)_{\text {tail }}$. Upper limits to the equivalent width are indicated as "U." The red symbols with dashed error bars correspond to the low-reddening sample, while the blue symbols with solid error bars show all the other SNe in our sample with measurements of $E(B-V)_{\text {tail }}$ and the Na I D equivalent width (or upper limit). 


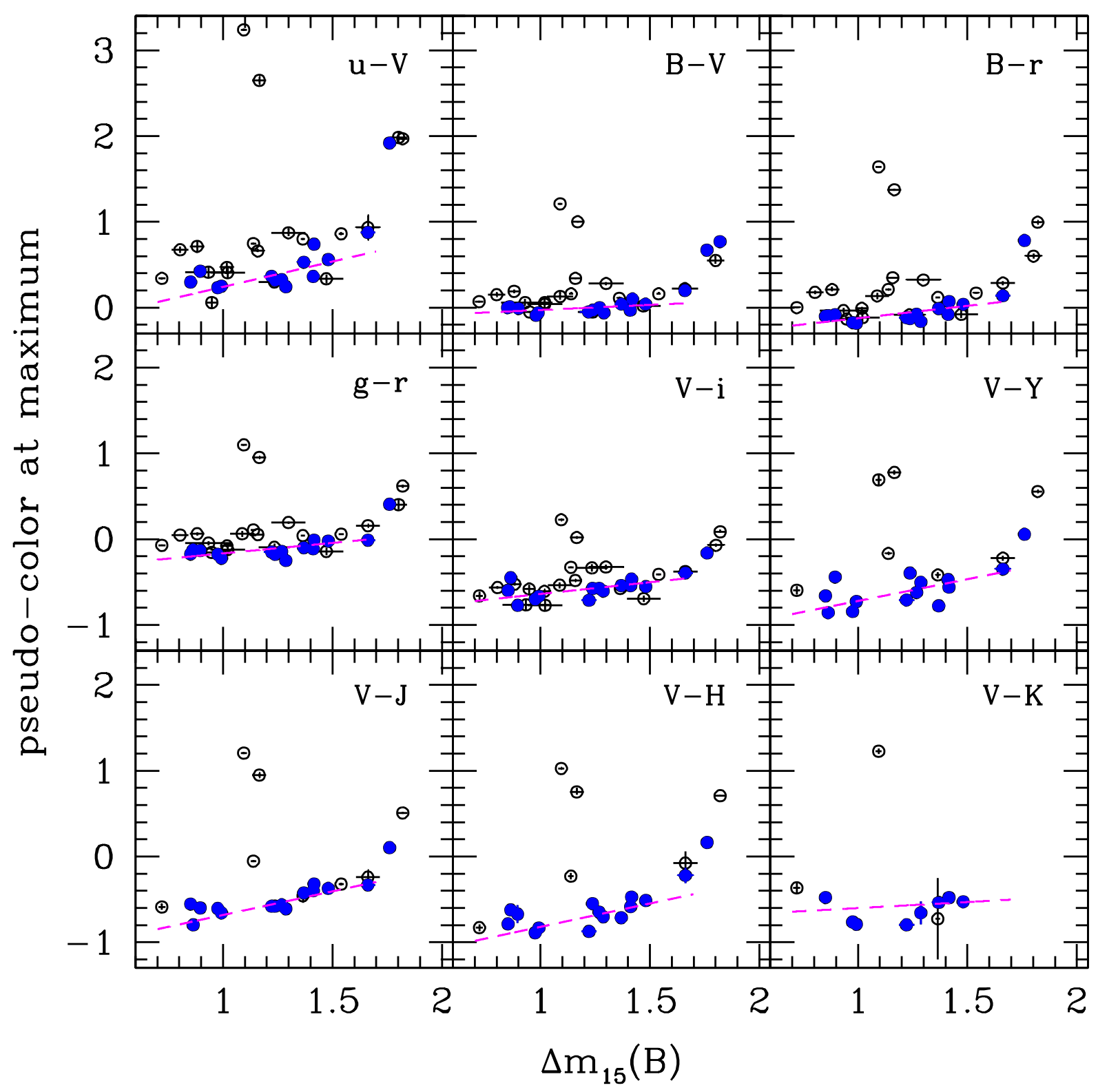

Fig. 11. - Pseudocolors at maximum light corrected for Galactic reddening, as a function of $\Delta m_{15}(B)$, for several filter combinations. Filled circles correspond to the low-reddening subsample of $\mathrm{SNe}($ see $\S$ 3.1) . The dashed lines are linear fits to the data of the low-reddening $\mathrm{SNe}$ in the range of $0.8<\Delta m_{15}(B)<1.7$ mag. 

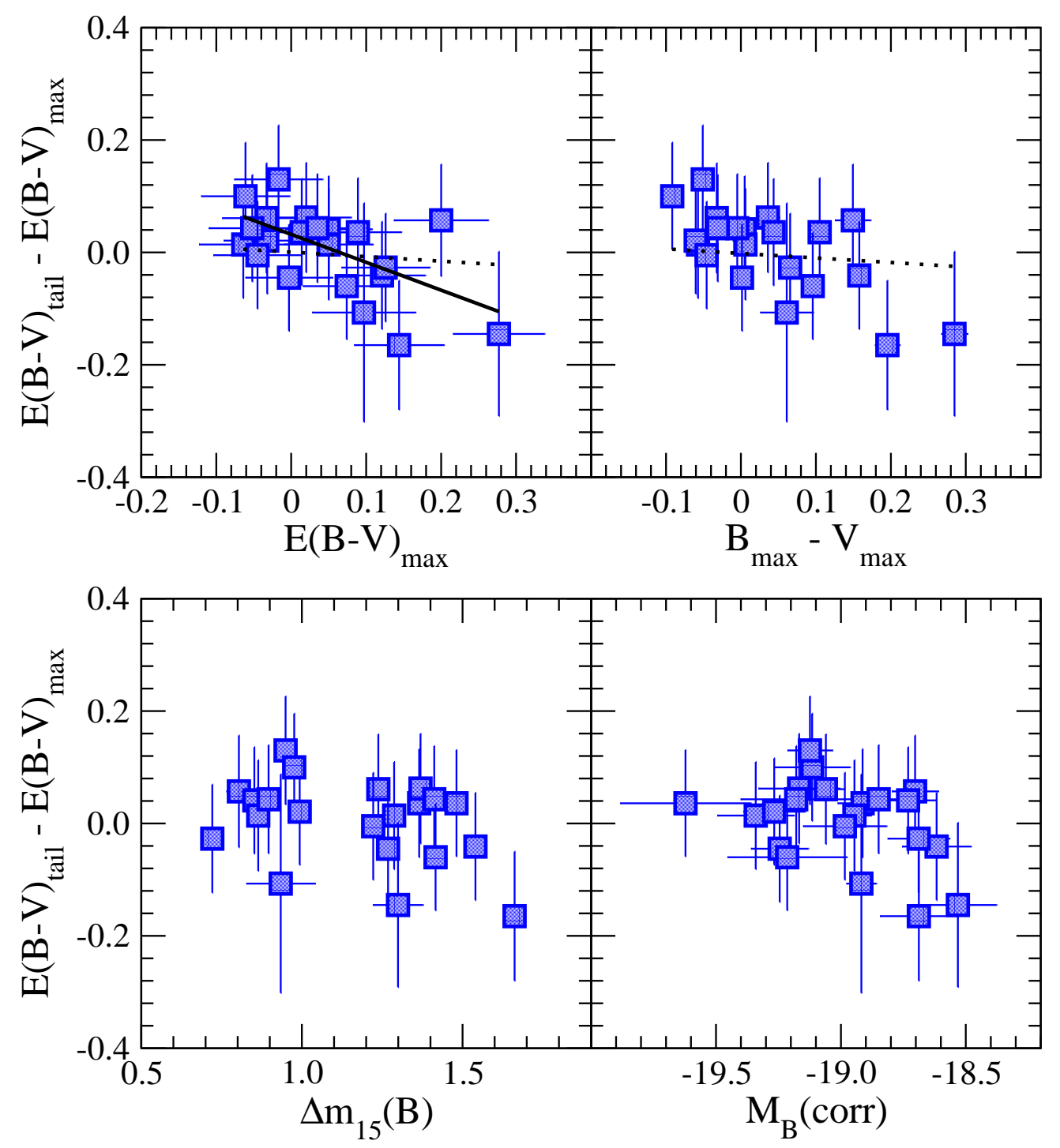

Fig. 12.- Comparison of color-excess estimates for $B-V$ from measurements at maximum light $\left[E(B-V)_{\max }\right]$ and at late epochs $\left[E(B-V)_{\text {tail }}\right]$. The difference between these two quantities is plotted vs. $E(B-V)_{\max }, B_{\max }-V_{\max }, \Delta m_{15}(B)$, and the absolute magnitude in $B$ corrected for decline rate (using Fit 1 of Table 9, see $\S 4.2$ ). The dotted lines in the top two panels indicate the weak correlations that would be expected due to the fact that the color excesses given in this paper are "observed" [as opposed to "true" color excesses (see Phillips et al. 1999)], and are measured at very different epochs in the color evolution of the SN. 


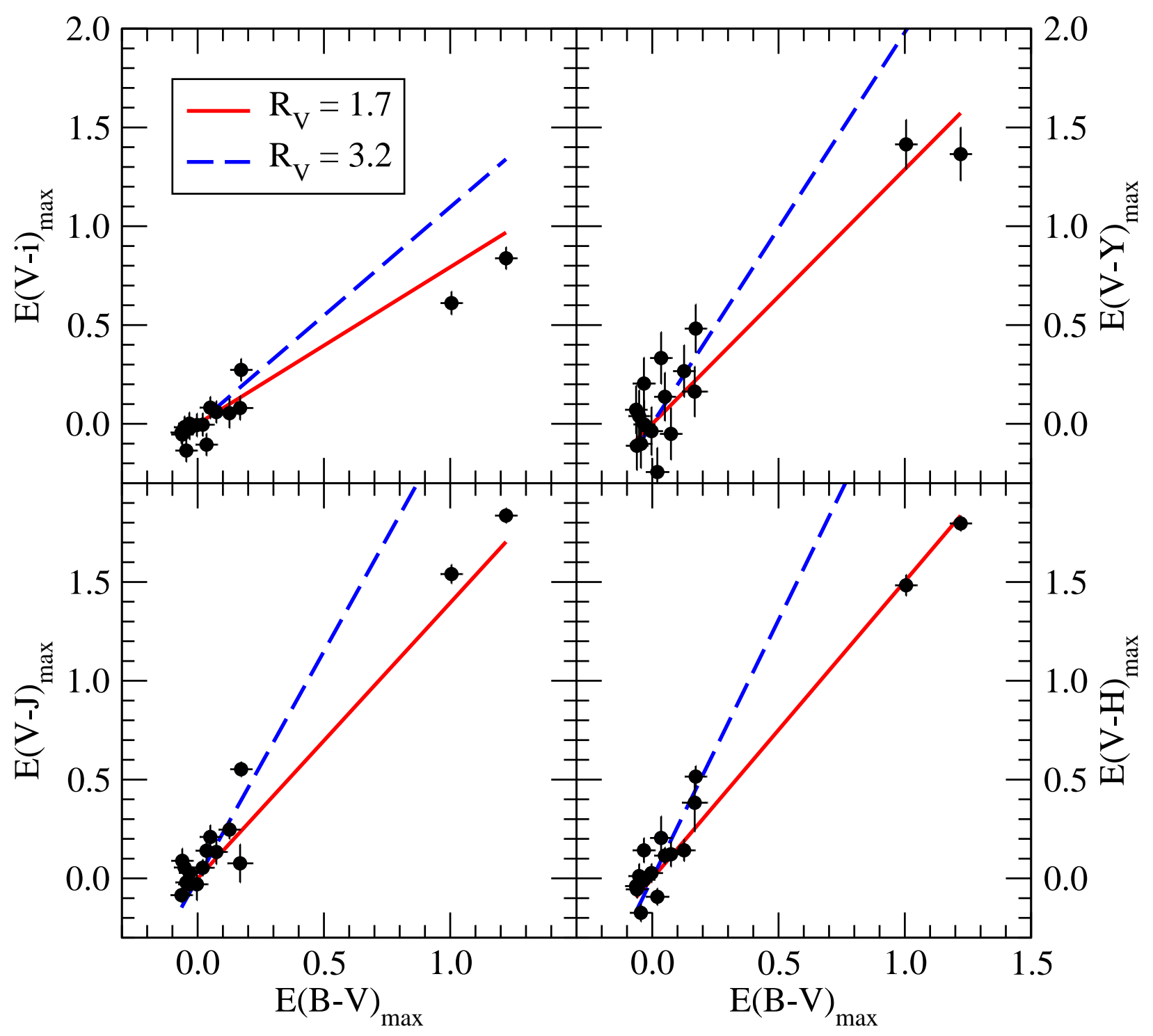

Fig. 13. - Comparison of color-excess estimates $E(V-X)_{\max }$, for $X \equiv i Y J H$, with $E(B-V)_{\max }$ for the best-observed SNe. The solid red lines represent the slope which corresponds to the average fit value of $R_{V}=1.7$, found using the whole set of points. The dashed blue lines indicate the slope predicted by a value of $R_{V}=3.2$, which is the averaged fit value found when excluding the two highly reddened $\mathrm{SNe}$ (the two points farthest to the right in the plots.) 


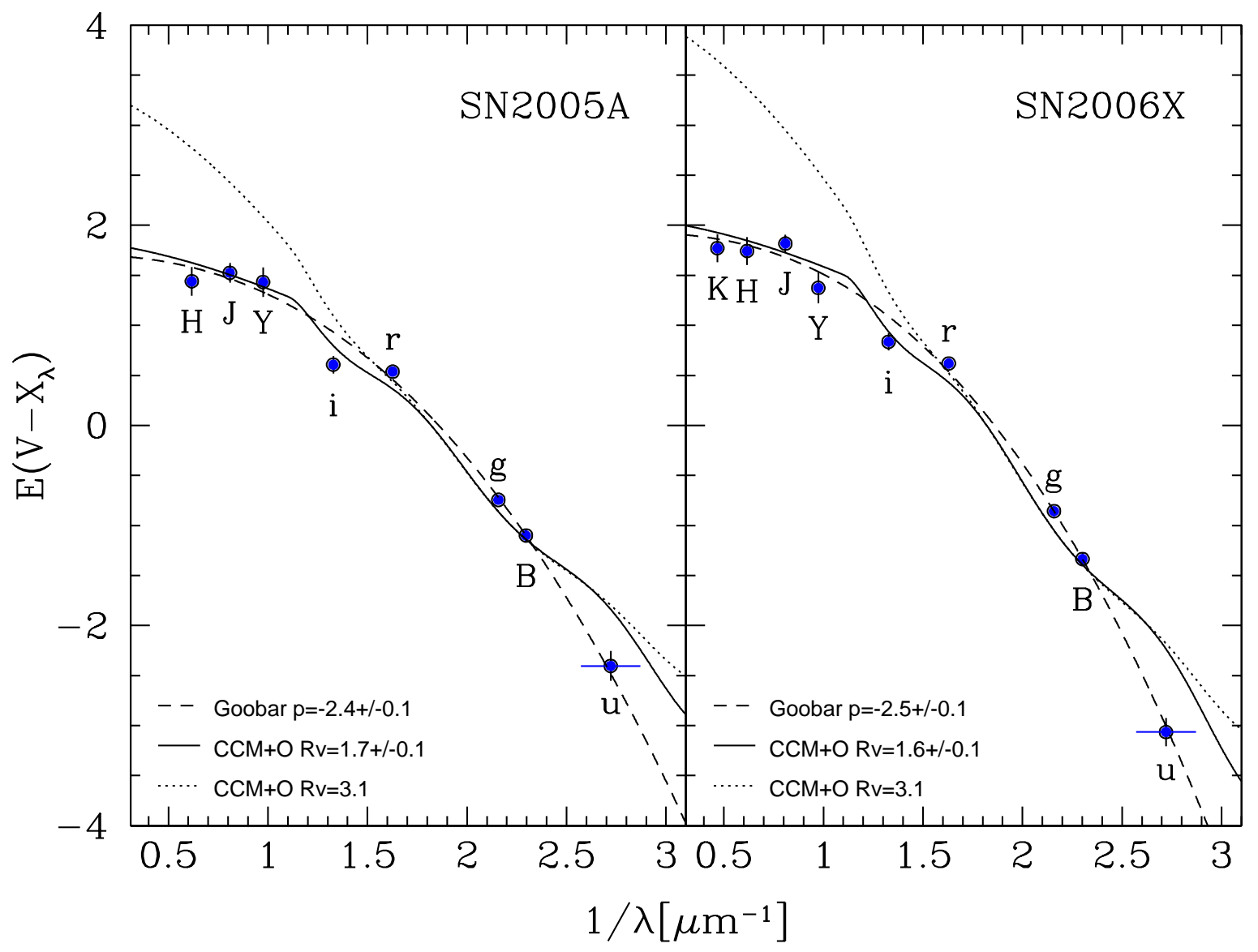

Fig. 14. - Color excesses $E\left(V-X_{\lambda}\right)$, for bands $X_{\lambda}=u g r i B Y J H K_{s}$, for SN 2005A (left panel) and SN 2006X (right panel). The solid lines show the best-fit CCM+O laws, whereas the dotted lines show the $\mathrm{CCM}+\mathrm{O}$ model for $R_{V}=3.1$. The dashed lines correspond to fits of the power-law model by Goobar (2008), valid for LMC-type dust. The latter provides a substantially better fit of the $u$-band reddening for both $\mathrm{SNe}$, as compared to the $\mathrm{CCM}+\mathrm{O}$ model. 


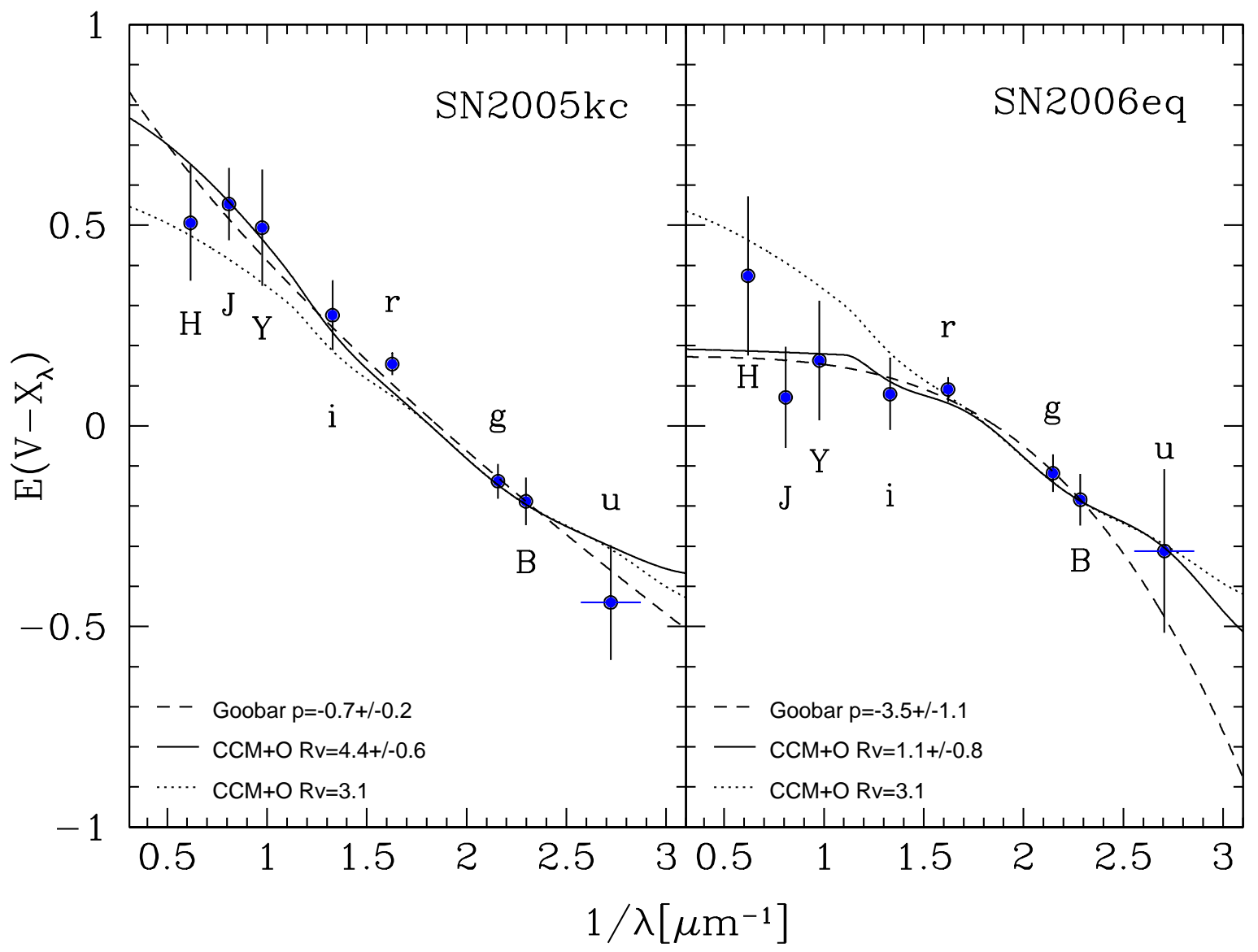

Fig. 15.- Color excesses $E\left(V-X_{\lambda}\right)$, for bands $X_{\lambda}=$ ugriBY JHK $K_{s}$, for SN 2005kc (left panel) and SN 2006eq (right panel). See the Figure 14 caption for further details. 

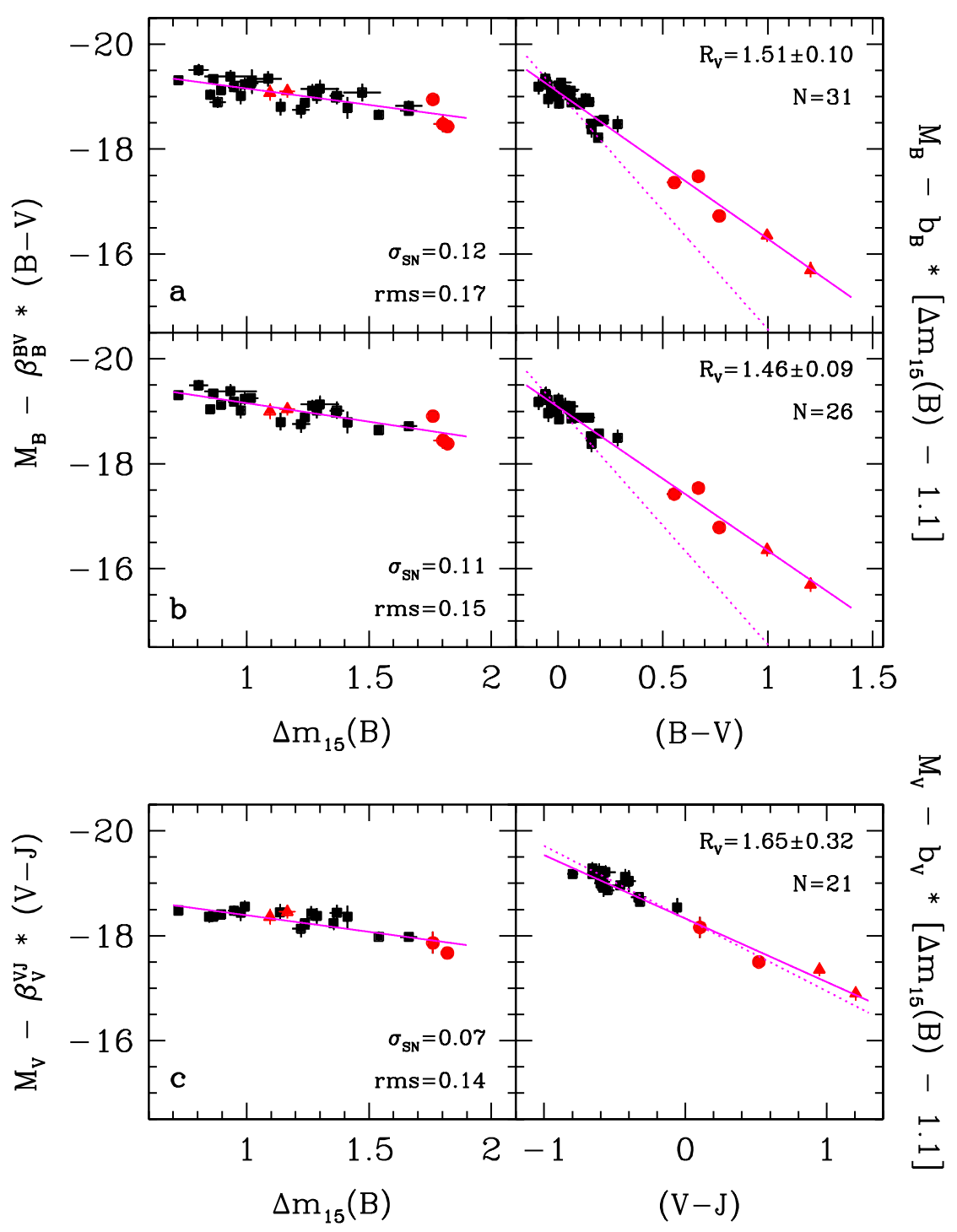

Fig. 16. - Sample fits of absolute peak magnitude versus decline rate $\left(\Delta m_{15}(B)\right)$ and color for SNe with reliable distances. One fit per row is displayed. The left panels show the relations between color-corrected absolute peak magnitude and decline rate. The right panels show the absolute peak magnitude vs. color relations, corrected for decline rate. Red circles denote the fast-declining SNe; red triangles mark the very red SNe 2005A and 2006X. The resulting fits are shown with solid lines. The dotted lines in these panels show the relations predicted by reddening with the CCM+O law and $R_{V}=3.1$. The number of SNe used in the fit, the rms scatter, the intrinsic dispersions $\left(\sigma_{\mathrm{SN}}\right)$, and the predicted $R_{V}$ are labeled. Rows: (a) $B$ vs. $\Delta m_{15}(B)$ and $B-V$ for the whole sample; (b) $B$ vs. $\Delta m_{15}(B)$ and $B-V$ for the subsample of best-observed SNe; (c) $J$ vs. $\Delta m_{15}(B)$ and $V-J$ for the subsample of best-observed SNe. 

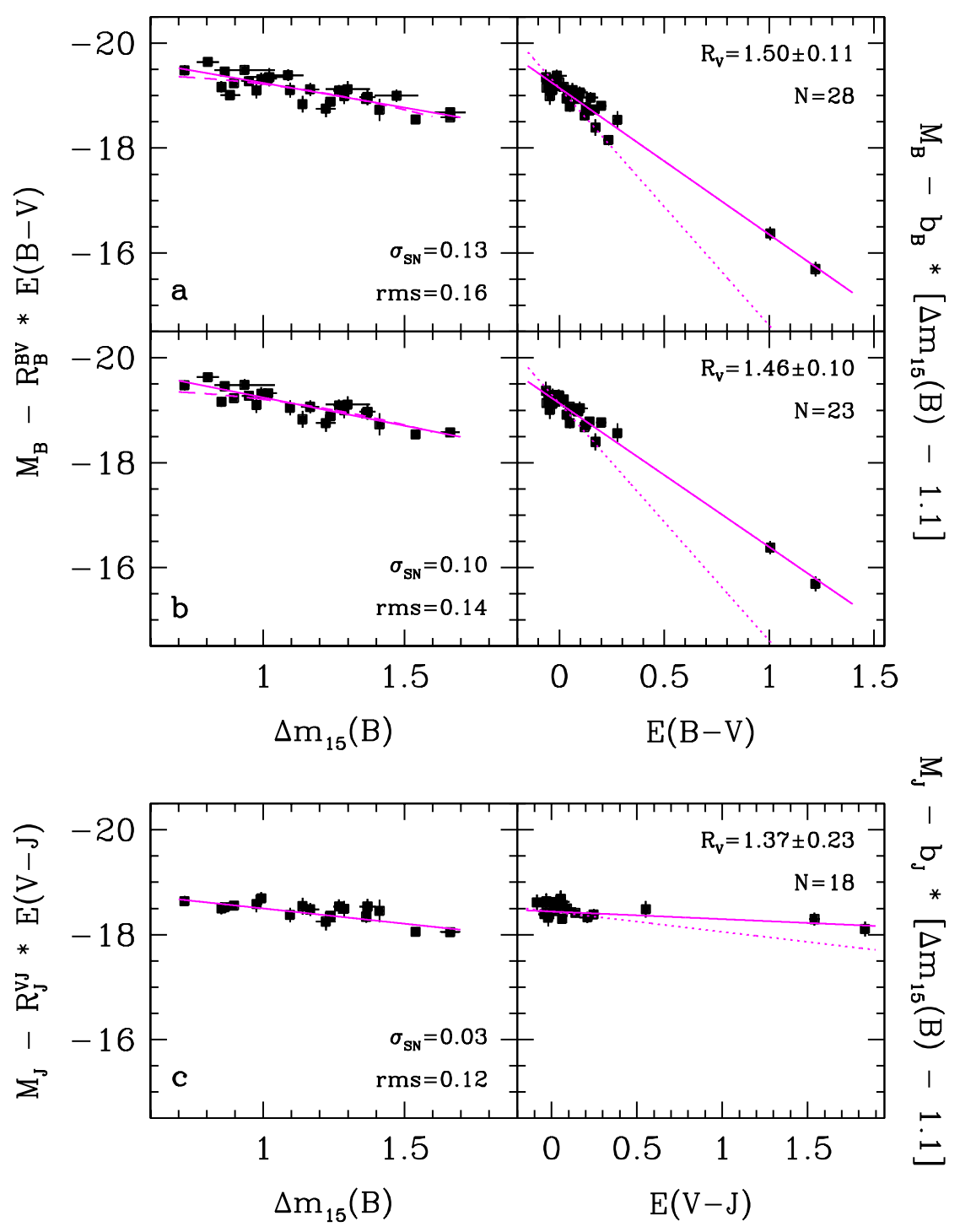

Fig. 17. - Fits of absolute peak magnitude versus decline rate $\left(\Delta m_{15}(B)\right)$ and reddening (measured as a color excess). One fit per row is displayed. The left panels show the relations between reddening-corrected absolute peak magnitude and decline rate. The right panels show the absolute peak magnitude vs. color excess relations, corrected for decline rate. The symbols for points and lines, and the labels, are equivalent to those used in Figure 16. For comparison, we added to the left panels of the upper two rows a dashed line indicating the quadratic relation fits by Phillips et al. (1999), shifted to the distance scale adopted in this work which corresponds to $H_{0}=72 \mathrm{~km} \mathrm{~s}^{-1}$ $\mathrm{Mpc}^{-1}$. Note that fast-declining SNe are not included in these plots. Rows: (a) $B$ vs. $\Delta m_{15}(B)$ and $E(B-V)$ for the whole sample; (b) $B$ vs. $\Delta m_{15}(B)$ and $E(B-V)$ for the subsample of best-observed SNe; (c) $J$ vs. $\Delta m_{15}(B)$ and $E(V-J)$ for the subsample of best-observed SNe. 


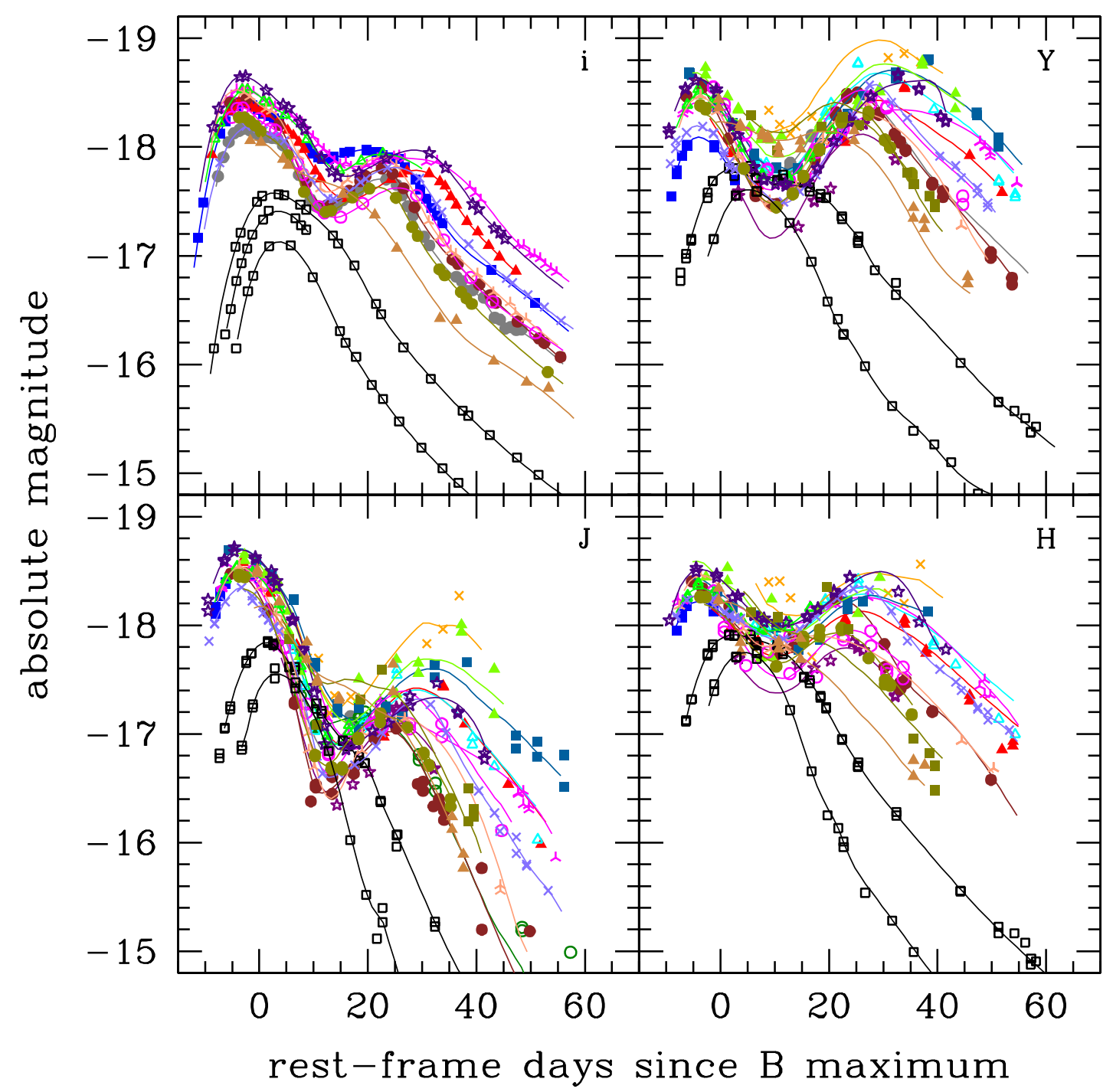

Fig. 18.- Absolute $i Y J H$ light curves for the subsample of best-observed SNe. The observed magnitudes are corrected for K-corrections, extinction in the Galaxy (assuming $R_{V}=3.1$ ), and extinction in the host galaxies (using the $R_{X}^{Y Z}$ values of Fits. 11 to 14). The times of observation are put in the rest frame of the $\mathrm{SNe}$, using the heliocentric redshifts, and referred to the time of $B$ maximum. The distances to the $\mathrm{SNe}$ are determined as described in $\S$ 4. The data from different $\mathrm{SNe}$ are shown with different symbols and the template light-curve fits are included for guidance. 


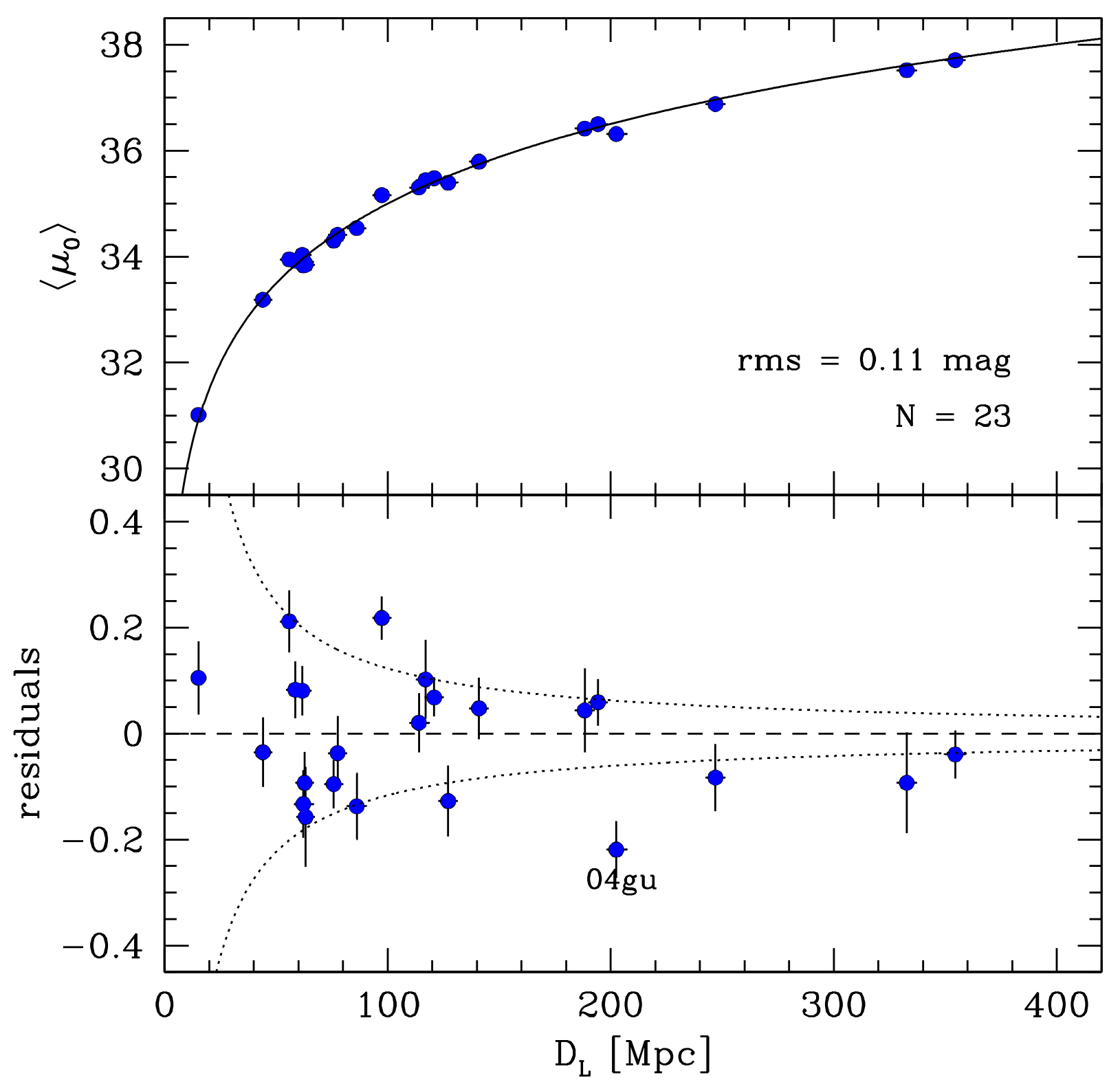

Fig. 19. - Hubble diagram for the sample of best-observed SNe out to $z \approx 0.08$. The top panel shows the combined distance moduli for each SN from the fits of absolute peak magnitude vs. decline rate and reddening in ugriBVYJH. The solid line shows the adopted concordance model of equation (5). The dotted lines represent the spread predicted by a dispersion of $382 \mathrm{~km} \mathrm{~s}^{-1}$ in the measured redshifts due to peculiar motions of the host galaxies. The bottom panel shows the residuals with respect to the model fit. 


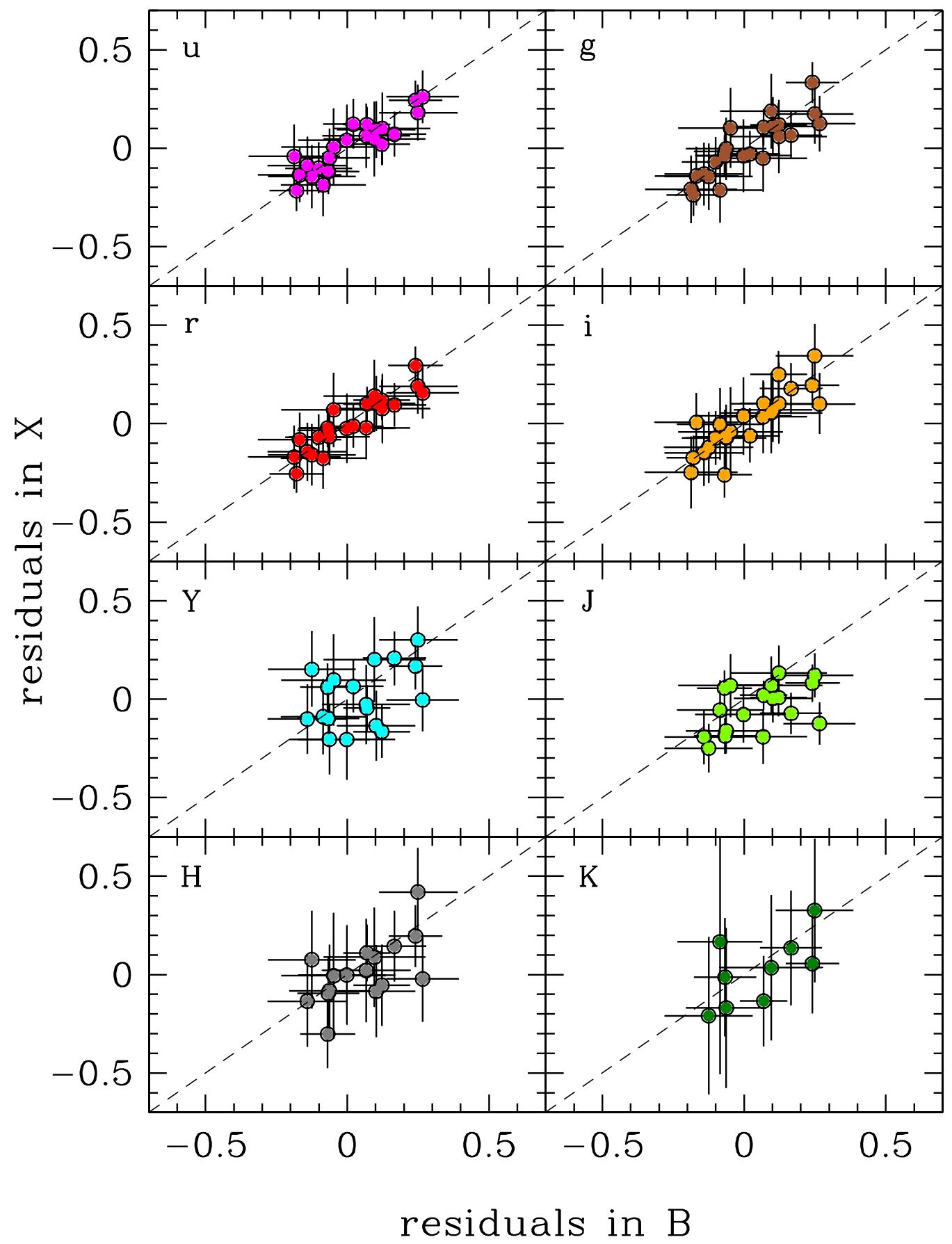

Fig. 20. - Residuals in the distance moduli calculated in band $X$, where $X=$ ugriY JHK, plotted versus the residuals in the $B$-band distance moduli. Note the significant correlation between these. 